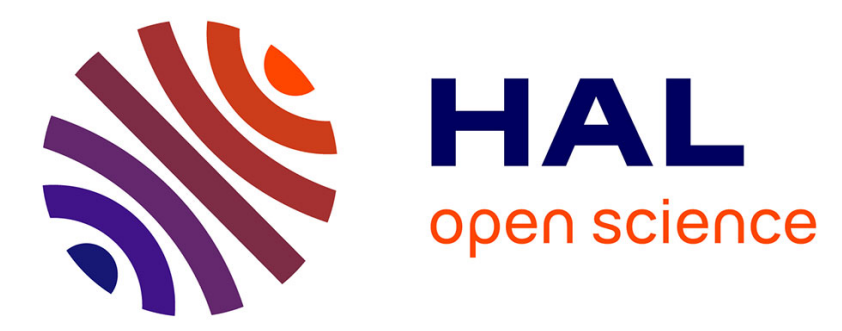

\title{
Electrocatalysis with single metal atom sites in doped carbon matrices
}

\author{
Tristan Asset, Frédéric Maillard, Frederic Jaouen
}

\section{To cite this version:}

Tristan Asset, Frédéric Maillard, Frederic Jaouen. Electrocatalysis with single metal atom sites in doped carbon matrices. Wiley. Supported Metal Single Atom Catalysis, , 2022. hal-03371171

\section{HAL Id: hal-03371171 \\ https://hal.science/hal-03371171}

Submitted on 8 Oct 2021

HAL is a multi-disciplinary open access archive for the deposit and dissemination of scientific research documents, whether they are published or not. The documents may come from teaching and research institutions in France or abroad, or from public or private research centers.
L'archive ouverte pluridisciplinaire HAL, est destinée au dépôt et à la diffusion de documents scientifiques de niveau recherche, publiés ou non, émanant des établissements d'enseignement et de recherche français ou étrangers, des laboratoires publics ou privés. 


\title{
Electrocatalysis with single metal atom sites in doped carbon matrices
}

\author{
T. Asset ${ }^{1}$, F. Maillard ${ }^{1}$, F. Jaouen ${ }^{2 *}$
}

${ }^{1}$ Institut Charles Gerhardt Montpellier, UMR CNRS 5253, Agrégats, Interfaces et Matériaux pour l'Energie, Université de Montpellier, 34095 Montpellier Cedex 5, France

${ }^{2}$ Université Grenoble Alpes, CNRS, Grenoble-INP, Université Savoie-Mont-Blanc, LEPMI, 38000 Grenoble, France

*Corresponding Author: Email.: frederic.jaouen@umontpellier.fr

Keywords: Electrocatalysis; Non-Precious Metal Catalysts; Oxygen Reduction; Carbon Dioxide Reduction; Single Metal Atom.

\begin{abstract}
While supported metal nanoparticles cannot achieve full electrochemical utilisation of metal atoms, catalysts featuring single metal atom sites may offer this possibility, along with advantages in selectivity. However, the passage from nanometric to atomic dimension is not without consequences. It first raises the question of efficient and robust synthesis methods, and underlines the need of cutting-edge characterization techniques that can target single metal atoms. These analytical tools are also pivotal to gain insights into the structure of the active sites, and establish atomic structure-catalytic activity-selectivity-stability relationships. Herein, we illustrate these topics for electrocatalysis, with a particular focus on metal-nitrogen-carbon single metal atom catalysts, for which a fantastic leap forward has been achieved in the last 15 years, triggered by the growing interest in sustainable energy storage and conversion systems.
\end{abstract}




\section{Introduction}

Electrochemical reactions take place at an electrode $\mid$ electrolyte interface. If the electrode material accelerates the rate of the electrochemical reaction, the phenomenon is referred to as 'electrocatalysis'. Electrochemical potential $(E)$ and current density $(j)$, two easily accessible parameters, are used in electrocatalysis to assess the performance of catalytic materials with socalled polarisation curves, $E=\mathrm{f}(j)$. In addition to temperature and pressure effects encountered in heterogeneous catalysis, the electrochemical potential is a key parameter in electrocatalysis, which can be used to dramatically increase the reaction rate of thermodynamically favourable reactions (e.g. in fuel cells), or, to force the occurrence of thermodynamically unfavourable reactions (e.g. electrolysis). Very important fields of application of electrocatalysis are the conversion of electrical energy into chemical energy and of chemical energy into electrical energy. Major examples of the former are water electrolysis, and co-electrolysis of water and carbon dioxide $\left(\mathrm{CO}_{2}\right)$, or water and nitrogen $\left(\mathrm{N}_{2}\right)$. In these devices, the following reactions need to be catalysed: hydrogen evolution reaction (HER) and oxygen evolution reaction (OER) in water electrolyzers, selective electroreduction of $\mathrm{CO}_{2}\left(\mathrm{CO}_{2} \mathrm{RR}\right)$ into $\mathrm{C}_{1}$ [formic acid $(\mathrm{HCOOH})$, carbon monoxide $(\mathrm{CO})$, and methane $\left.\left(\mathrm{CH}_{4}\right)\right]$ and $\mathrm{C}_{2}$ products (ethylene, ethanol) or the electroreduction of $\mathrm{N}_{2}$ in $\mathrm{NH}_{3}\left(\mathrm{~N}_{2} \mathrm{RR}\right)$. For chemical to electric energy conversion in electrochemical devices, fuel cells fed with $\mathrm{H}_{2}$ fuel are the most prominent example, and the reactions to be catalysed are the hydrogen oxidation reaction (HOR) and the oxygen $\left(\mathrm{O}_{2}\right)$ reduction reaction (ORR), even if other fuels have been or are being investigated, such as methanol, ethanol, or hydrazine.

The development of fuel cells began in the 1950-1960s for manned space missions. Attention focused onto low temperature fuel cells in the 1990s due to the growing need of carbon-free power sources. In alkaline fuel cells, many metals including non-precious metals are thermodynamically stable in their metallic, oxide or hydroxide form, and electrochemical reactions kinetics are usually more facile than in acidic media, although with the exception of the HOR. In acidic fuel cells (i.e. PEMFC - proton-exchange membrane fuel cell), the most industrially mature so far, only few metals are stable and active, typically platinum $(\mathrm{Pt})$ and platinum group metals (PGM, comprising platinum, iridium, osmium, palladium, rhodium, and ruthenium). Today, the state of art catalysts for the ORR and the HOR in PEMFCs are Pt-based nanoparticles 
(NP) supported onto high surface area carbon (Pt/C). The Pt loading in PEMFCs has been decreased radically from the 1980 's from $c a 10 \mathrm{mgPt}_{\mathrm{Pt}} \mathrm{cm}^{-2}$ geo to $c a 0.2 \mathrm{mgt}_{\mathrm{Pt}} \mathrm{cm}^{-2}$ geo today, by adding Nafion ${ }^{\circledR}$ in catalytic layers to improve the electrochemical utilization, and by nanostructuring Pt. However, even with 2-3 nm Pt NP, less than ca $50 \%$ of the atoms are located on the surface. Intuitively, further increasing the utilization of the rare and expensive Pt in PEMFC (especially for the ORR, since the HOR in acid medium requires only a low amount of Pt in PEMFC) may have been expected via the development of catalysts comprising single Pt atom sites. However, it is for the chlorine evolution reaction [1] and the HER [2-4] that promising single metal atom catalysts (SMAC) involving PGMs have been developed. The main reason behind this is the high surface energy of single metal atoms (SMA), which requires strong interactions with the support to avoid migration and aggregation. Metal oxides are well suited to strongly bind PGM-based SMA, but most of them are insulating or, in the best case, semiconducting, which is not desired for electrochemical applications targeting high $j$. Furthermore, the Gibbs-Thompson relation predicts that the redox potential of NP is inversely proportional to the particle size and shifts towards negative potentials with respect to the bulk material. As an example, the Gibbs-Thompson equation (with $V_{\mathrm{m}, \mathrm{Pt}}=9.110^{-6} \mathrm{~m}^{3} \mathrm{~mol}^{-1}$ the volume of a Pt atom [5] and a surface tension of $\gamma_{\mathrm{Pt}}=2.370 \mathrm{~J} \mathrm{~m}^{-2}$ [6]) predicts that single Pt atoms are thermodynamically under corrosion situation at any ORR-relevant potential in acidic solutions. Finally, the binding strength of oxygen intermediates is strongly correlated to the Pt-Pt coordination number, e.g. Pt with low coordination number $(\mathrm{CN}=1-2)$ and therefore closer to Pt SMAC exhibit a $\mathrm{OH}_{\text {ads }}$ adsorption strength which is $c a 1$ electronvolt $(\mathrm{eV})$ higher than optimal [7].

In contrast to single PGM atoms embedded in a carbon-based matrix, SMAC using non-precious metals embedded in the carbon structure have been successfully developed and demonstrated to catalyse the ORR. Early findings from Jasinski in 1964 that macrocyclic compounds based on $3 \mathrm{~d}$ transition metal [such as chromium $(\mathrm{Cr})$, manganese $(\mathrm{Mn})$, iron $(\mathrm{Fe})$, cobalt $(\mathrm{Co})$, nickel $(\mathrm{Ni})$, copper $(\mathrm{Cu})$ or zinc $(\mathrm{Zn})]$ and nitrogen $(\mathrm{N})$ with a Metal-N 4 configuration catalyse the ORR activity in both alkaline [8] and acidic [9] electrolyte initiated the development of SMAC in electrocatalysis. Catalysts were first synthesized by adsorbing $\mathrm{Fe}$ and Co macrocycles with well-defined square-planar Metal- $\mathrm{N}_{4}$ configuration onto a carbon support. In a second step, mild annealing $\left(500{ }^{\circ} \mathrm{C}\right)$ or higher temperature pyrolysis was shown to increase the stability of such catalysts, but questioned the nature of the active sites after the thermal treatment. A first 
breakthrough towards materials with SMA sites embedded into a nitrogen-doped carbon matrix (Metal-N-C) was achieved in 1989 by Gupta et al. who revealed that catalysts with ORR activity could be synthesized by pyrolysing a mix of metal, nitrogen and carbon precursors, with no Metal- $\mathrm{N}_{4}$ coordination pre-existing the pyrolysis [10]. A second breakthrough was made in 2009-2011: for the first time, ORR performance compatible with PEMFC application was demonstrated for Fe-N-C materials $[11,12]$. The introduction of thermally decomposable metalorganic frameworks (MOFs) as a carbon and nitrogen source further improved the ORR activity compared to the existing state of art, and more importantly, improved the accessibility of the reactants to the active sites $[13,14]$. This chapter focuses on Metal-N-C SMAC involving one or several $3 \mathrm{~d}$ transition metal, since they are scientifically and technologically the most mature SMAC for application in various electrochemical devices. For completeness and outlook into the future, we also shortly discuss latest reports on carbon-embedded SMAC prepared with other metals and/or other light-element dopant (e.g. Pt-S-C and tin (Sn)-doped N-C). This review chapter is organized as follows. In Section 13.2, we describe how these materials can be synthesized for application in electrocatalysis. In Section 13.3, the focus is on the structure of the active sites for electrocatalytic reactions, and key physico-chemical techniques that have been used to characterize these materials. When available, we detail how these techniques can be used in situ/operando. Section 13.4 is devoted to the mechanisms of the ORR, the $\mathrm{CO}_{2} \mathrm{RR}$ and the $\mathrm{N}_{2} \mathrm{RR}$ or the $\mathrm{NO}_{3}$ reduction reaction $\left(\mathrm{NO}_{3} \mathrm{RR}\right)$ on such materials. As research has recently moved to the next important step for application, namely stability and degradation mechanisms of Metal-N-C catalysts, we cover these aspects in section 13.5. Finally, Section 13.6 gives conclusions and an outlook in the future of carbon-embedded SMAC.

\section{Synthesis methods}

Before the advent and broad availability of characterization techniques that can unambiguously identify SMA sites (e.g. X-ray absorption spectroscopy (XAS), high-resolution scanning transmission electron microscopy (HR-STEM), and cryostatic ${ }^{57} \mathrm{Fe}$ Mössbauer spectroscopy), the synthesis of Metal-N-C SMAC by pyrolysis was an intention rather than a demonstrated fact. The initial reports on the ORR activity of unpyrolysed macrocycles $[8,15,16]$ revealed, that $\mathrm{Fe}$ and Co were, by far, the most active ones among $3 \mathrm{~d}$ transition metals for catalysing the ORR, both in alkaline and acidic media. This important learning was found to be valid for Metal-N- 
C SMAC prepared via pyrolysis in the following decades. Hence, we focus mainly on the synthesis methods of Fe- and Co-N-C SMAC in this section. While in those early days, the use of Metal-N 4 macrocycles without pyrolysis secured the well-defined Metal-N 4 active centres, the different approaches used to interface them with a conductive support impacted the local environment of metal cations and their accessibility. Phthalocyanines and porphyrins are poorly soluble in water, and different solvents or suspensions in concentrated sulphuric acid were used, removing the solvent by evaporation or precipitating the macrocycle on the support. The dispersion quality of metal macrocycles on supports were shown to depend strongly on the morphology, specific surface area (SSA) but also on acido-basic properties of carbon supports $[17,18]$. For example, the characterization by ${ }^{57} \mathrm{Fe}$ Mössbauer spectroscopy, a technique which allows identifying the different coordinations and spin states of Fe (discussed in more detail in 13.3), of iron phthalocyanine (Fe-Pc) precipitated on a carbon powder revealed multiple coordinations and spin states for $\mathrm{Fe}$, whereas a single coordination and electronic state was seen for the Fe-Pc monomer [17]. Controlled deposition or solvent removal however resulted in $\mathrm{Fe}-\mathrm{Pc} / \mathrm{C}$ composites with a single (or vast majority of one) ${ }^{57} \mathrm{Fe}$ Mössbauer spectroscopic signature [19]. For example, removal of the pyridine solvent for Fe-Pc by boiling it off at $420^{\circ} \mathrm{C}$ resulted in a $\mathrm{Fe}-\mathrm{Pc} / \mathrm{C}$ composite with $95 \%$ of the spectral signal assigned to one specific doublet [19]. The latter can nowadays be assigned to an $\mathrm{O}_{2}-\mathrm{Fe}(\mathrm{III})-\mathrm{N}_{4}$ coordination [20], implying high dispersion and accessibility to $\mathrm{O}_{2}$ of the $\mathrm{Fe}-\mathrm{N}_{4}$ sites.

While some heterogeneities of metal coordination and site accessibility were exemplified above for SMAC prepared via interfacing Metal-N 4 macrocycles without high-temperature pyrolysis, the extent of heterogeneities was progressively increased, and the true nature of the active site blurred for several decades, with the introduction of high-temperature treatments of macrocycles in a first step, and the switch to the synthesis of Metal-N-C SMAC from separate metal, nitrogen and carbon precursors at high temperature, in a second step. Mild (200-500 $\left.{ }^{\circ} \mathrm{C}\right)$ and then more aggressive thermal treatment $\left(>600^{\circ} \mathrm{C}\right)$ in inert or reductive atmosphere was applied to Metal-N4 macrocycles supported on carbon (labelled henceforth as Metal-N4/C), starting in 1976 [15]. This was triggered in response to the quickly decaying ORR activity of non-heattreated Metal- $\mathrm{N}_{4} / \mathrm{C}$ materials in acidic medium, in an attempt to improve their lifetime. Improved electrochemical durability in acid medium was always observed after pyrolysis, while the magnitude of increase in the ORR activity highly depended on the nature of the metal (Fe, 
Co) and of the macrocycle (phthalocyanines, porphyrins, tetraazaannulene, etc.) [21-26]. As a general rule, the improvement in ORR activity was however $>20$ times. The effect of the annealing temperature on a Co-Pc/C material was studied, for example, with Raman spectroscopy, showing that the peaks characteristic for pristine Co-Pc disappeared after a pyrolysis at $700{ }^{\circ} \mathrm{C}$ [27]. The transformation was proposed to be the loss of peripheral functional groups first while the Co-N 4 core was retained, and then above $700{ }^{\circ} \mathrm{C}$ the complete disintegration of the phthalocyanine $(\mathrm{Pc})$ structure. The fate of the metal ions and their coordination after a high-temperature treatment of Metal- $\mathrm{N}_{4} / \mathrm{C}$ materials has been the object of a long scientific debate that will only be shortly discussed here. The three main hypotheses on the nature of the active site resulting from pyrolysis were (i) Metal- $\mathrm{N}_{4}$ coordination after any pyrolysis temperature, similar to the core of macrocycles [28], (ii) a C-N $\mathrm{N}_{\mathrm{x}}-$ Metal complex, with the metal adsorbing on $\mathrm{C}-\mathrm{N}_{\mathrm{x}}$ moieties in situ during electrochemistry [10], (iii) the formation of specific Metal- $\mathrm{N}_{\mathrm{x}}-\mathrm{C}_{\mathrm{y}}$ site at high-temperature [18]. While unoptimized syntheses initially lead to the formation of metallic Co or Fe particles during pyrolysis (possibly along with SMA sites) [28,29], the application of specific techniques that can distinguish metal aggregates from SMA sites, correlation between the metal coordination, ORR activity and synthetic approach, resulted in the early 2000's in the identification of the existence of SMA sites of the type Metal- $\mathrm{N}_{\mathrm{x}}$, even after pyrolysis [30-32]. These sites should however be regarded as structurally and electronically different from Metal$\mathrm{N}_{4}$ sites in macrocycles, since the Metal- $\mathrm{N}_{\mathrm{x}}$ sites are covalently integrated in the carbon support (which we therefore refer henceforth to as a "carbon matrix", when the final material is discussed) after a pyrolysis at $700-1000{ }^{\circ} \mathrm{C}[32]$. The covalent integration of Metal- $\mathrm{N}_{\mathrm{x}}$ sites in the carbon matrix generally explains the improved stability and electrocatalytic activity relative to unpyrolysed Metal-N $\mathrm{N}_{4} / \mathrm{C}$ materials. The active site in such SMAC is no longer a molecule, but a moiety in a conductive material. The effect of the temperature and macrocycle loading on carbon supports was shown to impact the type of metal species present after pyrolysis [28,3335]. Excessive macrocycle loading leads to metal aggregation at high temperature, and their carbo-reduction. In turn, Fe and Co metallic particles catalyse the graphitization of the carbon support, which decreases the nitrogen content, accelerating the transformation of Metal- $\mathrm{N}_{\mathrm{x}}$ sites into metallic aggregates. Optimized macrocycle loading and initial dispersion, pyrolysis temperature and duration can however lead to Metal-N-C materials with all metal atoms as Metal$\mathrm{N}_{\mathrm{x}}$ sites [36]. The ORR performance of pyrolysed metal-macrocycle SMAC was greatly improved through the simultaneous removal of the carbon support and the addition of porogens. 
This approach was originally developed by the group of Tributsch and then continued by Kramm [37,38]. It however necessitates the careful removal by acid-wash of excessive metal after pyrolysis, a natural consequence of the high metal content in macrocycles (e.g. $10 \mathrm{wt}$ \% $\mathrm{Fe}$ in $\mathrm{Fe}-\mathrm{Pc}$ with molecular formula $\mathrm{C}_{32} \mathrm{H}_{16} \mathrm{FeN}_{8}$ ), well above the current limit of $\mathrm{Fe}$ as $\mathrm{Fe}-\mathrm{N}_{\mathrm{x}}$ sites in Fe-N-C materials ( $c$ a 2-3 wt. \% Fe). The addition of sulphur (S) before pyrolysis was shown to result in the formation of Fe or Co sulphide phases after a first pyrolysis, that can be easily acid-washed, leaving behind only the acid-stable Metal- $\mathrm{N}_{\mathrm{x}}$ sites. In addition, Fe and Co sulphides do not catalyse graphitization at high temperature, also helping in retaining a high nitrogen content, important for high number of Metal- $\mathrm{N}_{\mathrm{x}}$ sites. Nowadays, the Metal- $\mathrm{N}_{4}$ macrocyclic approach to prepare Metal-N-C SMAC is still an active field, with original approaches such as the preparation of MOF structures integrating porphyrins as building blocks [39,40], combining metal porphyrins with the silica hard-templating method [41] or forming aerogels of porphyrins [42] before pyrolysis.

While the clear experimental proof for the existence of ORR-active Metal- $\mathrm{N}_{\mathrm{x}}$ sites in Metal-N$\mathrm{C}$ materials derived from the pyrolysis of Metal-N 4 macrocycles was perhaps available only in the early 2000's, the idea emerged long before the proof was obtained, that perhaps all that is needed for driving the spontaneous formation of ORR-active sites is the simultaneous presence of metal (Fe, $\mathrm{Co}, \mathrm{Sn})$, carbon and nitrogen at high temperature. The first pyrolytic synthesis of a Metal-N-C material not resorting to a Metal-N 4 macrocycle was probably reported by Yeager's group in 1989 [10]. Polyacrylonitrile, Fe or Co acetate salt, and a carbon black powder were mixed by wet chemistry in dimethylformamide, then dried, and pyrolysed in inert gas. The samples showed no ORR activity after annealing at low temperature, and optimum ORR activity after annealing at 750 or $900{ }^{\circ} \mathrm{C}$, depending on the nature of the metal. This work opened the door to Metal-N-C synthesis from simple metal salts, nitrogen and carbon precursors. Between 1989 and 2009, most pyrolytic syntheses of Metal-N-C materials involved a pre-existing carbon support, adding metal precursors (most often, simply salts) and nitrogen precursors in optimized amounts [43-45]. The use of a pre-existing carbon support offered a reasonably good control of the morphology, graphitic ordering, porosity and SSA of the final catalyst. The disadvantages were the lack of "mobility" of carbon atoms from such a support (especially for the more graphitic carbon supports [46]), as well as propensity for metal aggregation into particles in the case of carbon supports with a low SSA. The carbon mobility aspect is important 
to explain at this stage. The site formation at high temperature requires the rearrangement of carbon, nitrogen and metal atoms. While the nitrogen-rich organic precursors and metal salts are highly reactive above $700{ }^{\circ} \mathrm{C}$, this is much less the case for carbon atoms from the carbon support due to the high melting point of graphite $\left(>3600^{\circ} \mathrm{C}\right)$. It was revealed in 2004-2006 that the presence of a disordered carbon phase in the starting carbon support is critical to reach high ORR activity of the resulting Fe-N-C materials [47-49]. It was also shown that a negative correlation exists between the SSA and the content of disordered carbon in carbon blacks. Since both high SSA and high content of disordered carbon are desirable to prepare a Metal-N-C catalyst precursor, the negative correlation mentioned above was a limiting factor for Metal-NC materials until 2009. This lead to (i) the parallel formation of metal particles and Metal- $\mathrm{N}_{\mathrm{x}}$ sites, (ii) a low density of Metal- $\mathrm{N}_{\mathrm{x}}$ active sites, and an overall unsatisfactory ORR activity and performance of Metal-N-C catalysts, with ORR activity of only $1-2 \mathrm{~mA} \mathrm{~cm}^{-2}$ geo at $0.9 \mathrm{~V}$ cell voltage $[47,50]$.

Another breakthrough was achieved in 2009, with a novel synthesis leaning on the functionalization of a high SSA carbon black, namely Black Pearls 2000 (BP2000), with an organic molecule rich in nitrogen and carbon, namely 1,10-phenanthroline [11]. Functionalizing the micropores of BP2000 with optimized amounts of phenanthroline and ferrous acetate resulted in a catalyst precursor with high amount of disordered carbon and optimized amounts of Fe and nitrogen. The pyrolysis of such catalyst precursors resulted in Fe-N-C materials achieving a

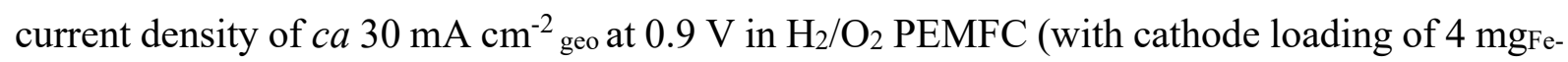
$\mathrm{N}-\mathrm{C} \cdot \mathrm{cm}^{-2}$ geo), similar to the value obtained with a state-of-art cathode with $0.4 \mathrm{mgPt}^{-2} \mathrm{~cm}_{\text {geo }}$ [11] In more detail, it was shown that the micropores of the high SSA support were filled with phenanthroline, and the pores re-opened during pyrolysis as a result of the transformation of phenanthroline and Fe salt into $\mathrm{Fe}-\mathrm{N}_{\mathrm{x}}$ sites, with $\mathrm{ca} 2 / 3^{\text {rd }}$ of the mass of phenanthroline leaving the reactor as volatile products. The concept of the preparation of catalysts precursors comprising "disordered carbon", nitrogen atoms and metal atoms was found to be valid for other high surface area carbon supports, including carbide-derived carbons [51,52], and for other organic molecules than phenanthroline [53]. One remaining drawback of the "functionalized carbon support approach" was the high relative amount of inactive carbon atoms in the catalyst. The idea therefore progressively emerged to design syntheses that do not lean on pre-existing carbon 
supports, but rather involve organic or metal-organic materials as carbon and nitrogen precursors. Beside the pore-forming agent approach already discussed earlier for preparing highly active Metal-N-C materials from metal-porphyrins, four other synthetic approaches are discussed below, that have found success in the preparation of high-performance Metal-N-C catalyst for ORR, leading ultimately to SMAC.

\subsection{Hard templating with silica}

In this approach, a macrocycle, or separate metal, nitrogen and carbon organic precursors, are mixed in optimized amounts with silica particles, and pyrolysed (Figure 1a). The removal of silica particles by hydrofluoric acid and/or potassium hydroxide $(\mathrm{KOH})$ leaves a Metal-N-C catalyst with high amount of mesopores by hard templating effect, with some micropores also present in the carbon phase resulting from the pyrolysis of the organic precursors. This approach was originally developed for Metal-N-C materials by Atanassov's group in 2008 [54], still resorting to Metal-porphyrins. More active Fe-N-C materials were prepared later by resorting to Fe salts and N-C precursors such as aminoantipyrine, nicarbazin, etc. [55-58]. In optimized conditions, the synthesis results in the majority or complete dispersion of $\mathrm{Fe}$ into $\mathrm{Fe}-\mathrm{N}_{\mathrm{x}}$ sites [56,59]. For optimized ORR activity, re-annealing the catalyst after silica removal is however important. The silica templating synthesis of Metal-N-C SMAC has been scaled-up by the company Pajarito Powder. The scaled-up material however shows a lower relative amount of Fe$\mathrm{N}_{\mathrm{x}}$ sites than laboratory batches, with concurrent metallic Fe particles (compare the PAJ and UNM samples in Ref. [59]). This gives an example of the challenge of scaling-up SMAC [59]. Interestingly, although the site density of $\mathrm{Fe}-\mathrm{N}_{\mathrm{x}}$ sites was low in the scaled-up material, their turnover frequency (TOF, number of electrons transferred per site per second) was high, leading to an overall competitive ORR activity. The particle size, morphology and SSA of the silica template was found to affect the resulting $\mathrm{Fe}-\mathrm{N}-\mathrm{C}$ catalysts [60]. An alternative use of silica was reported by Joo's group in 2016, by depositing a silica overlayer on top of carbon nanotubes that had been previously coated with a monolayer of Fe-porphyrin [61]. The work showed that the silica overlayer minimized the formation of Fe particles during pyrolysis, thereby increasing the final Fe- $\mathrm{N}_{\mathrm{x}}$ site density, and ORR activity. 

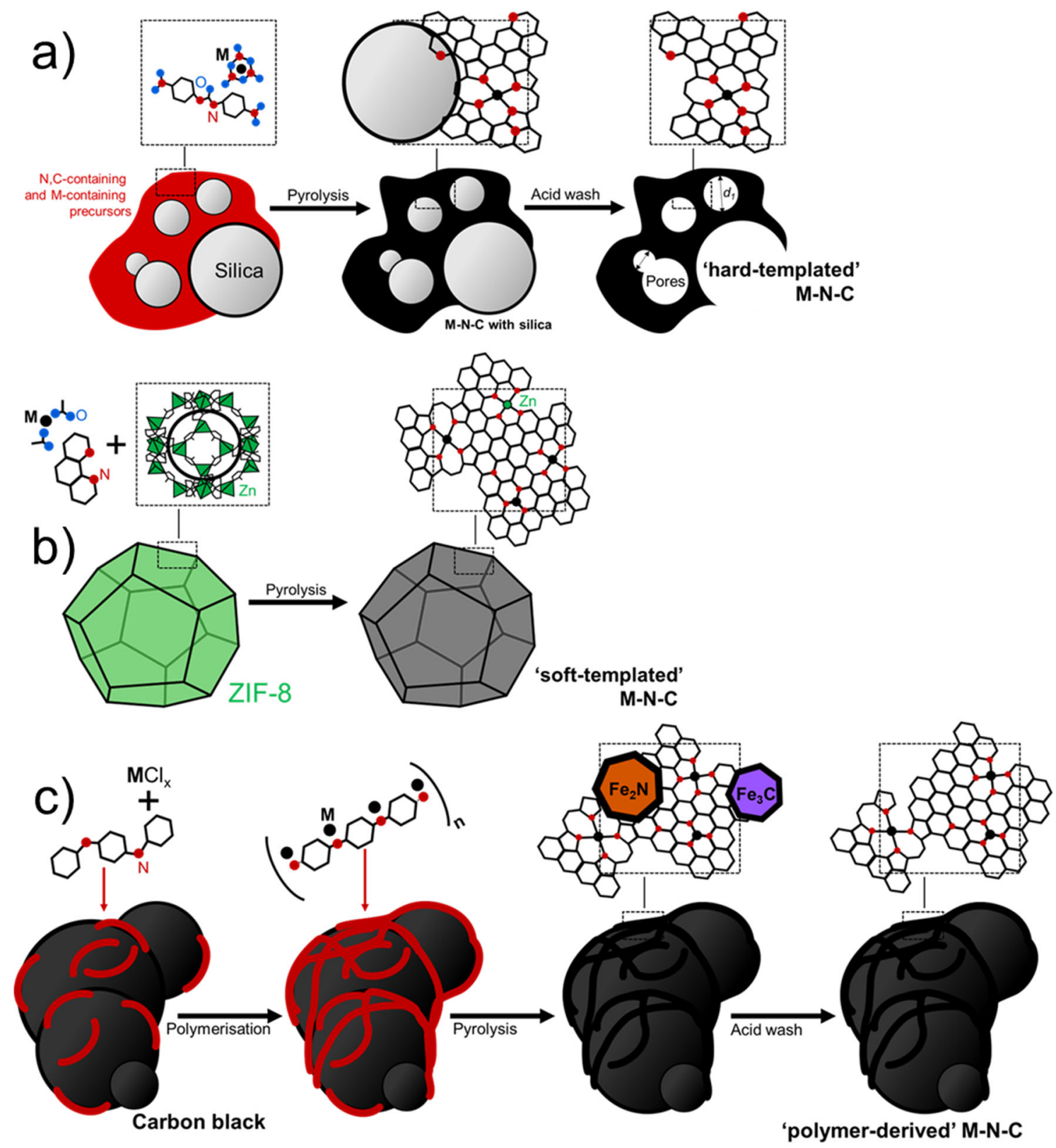

Figure 1. Main synthesis methods to achieve metal-nitrogen-carbon electrocatalysts: a) hard templating approach with silica as an example; b) soft-templating approach with ZIF-8 as an example; c) sacrificial polymer approach with polyaniline as an example - the red lines are representative of the aniline before and after polymerisation. Source: Tristan Asset, Frédéric Maillard, Frédéric Jaouen.

\subsection{Soft-templating with Metal-organic frameworks}

The use of MOFs for preparing Fe-N-C materials via pyrolysis was first reported in 2011, by the groups of Liu and Dodelet $[13,14]$. In Liu's conceptual approach, a Co-based zeolithic im- 
idazolate framework (ZIF) was a promising catalyst precursor, featuring a high density of tetrahedral Co- $\mathrm{N}_{4}$ coordination sites. Following pyrolysis at $750{ }^{\circ} \mathrm{C}$, the material showed promising ORR activity. The amount of Co in their ZIF ( $c a 30$ wt. \%) was however well above the limit that can be integrated as $\mathrm{Co}-\mathrm{N}_{\mathrm{x}}$ sites in Co-N-C. As a result, metallic Co NP formed during pyrolysis. In Dodelet's approach, MOFs were of interest as a highly and well-defined microporous substrate (micropores in Fe-N-C had previously been showed to correlate with high ORR activity by his group [62]), while simultaneously containing nitrogen and carbon atoms. The specific nature of the metal in the MOF was regarded as less important, since an optimized (small) amount of Fe or Co can be added as a salt, by wet chemistry approach, before pyrolysis. The Zn-based ZIF-8 structure was commercially available already in 2011, and this MOF was used in replacement of BP2000, in an otherwise similar synthesis as that reported in 2009 [11]. The resulting optimized Fe-N-C material, whose synthesis is illustrated in Figure 1b, showed $c a$ twice increased power performance in $\mathrm{H}_{2} / \mathrm{O}_{2}$ PEMFC compared to their report of 2009, reaching a peak power density of $0.9 \mathrm{~W} \mathrm{~cm}^{-2}$ geo [13]. This demonstrated improved accessibility of the Fe-based active sites with the sacrificial ZIF-8 approach. Scanning electron microscopy (SEM) images showed that the nitrogen-doped carbon matrix resulting from ZIF-8 pyrolysis had a defined geometry, with size and shape similar to that of the ZIF- 8 crystals before pyrolysis while TEM images showed that the catalytic particles contained interconnected pores [13]. The availability of a commercial Zn-based ZIF-8 in 2011 was fortuitous, and was later proved to be (near)optimal, with the easy removal of $\mathrm{Zn}$ during pyrolysis, due to its low boiling point of $907^{\circ} \mathrm{C}$. These two works from 2011 spurred an entire line of research on Metal-N-C and N-C materials derived from MOF pyrolysis (ZIF-8 in particular), as reviewed for example in Refs $[63,64]$. Subsequent works showed the importance of nanostructuring the ZIF crystals, leading to their soft-templating into nanosized Fe-N-C or Co-N-C particles with the same dodecahedron shape [65-67]. One-pot syntheses of Fe-doped ZIF-8 were then reported [68,69]. Other onepot syntheses were developed, with ZIF-8 acting as a host, and selecting Fe precursors small enough to be encapsulated in the inner cavity of ZIF-8 or other MOFs. This was achieved for example with the encapsulation of ferrocene in ZIF-8 [70] or of the Fe(phenanthroline)3 complex in the cavity of three different Zn-based ZIFs [71]. In the latter study, the ZIF with the largest cavity size led to the Fe-N-C with the highest SSA and highest ORR activity. Similarly, a comprehensive study on the preparation of Fe-N-C materials from different Zn-based ZIFs revealed the importance of the cavity size to reach high ORR activity of Fe-N-C materials after 
pyrolysis [72]. Despite numerous ZIFs and MOFs investigated to date, ZIF-8 remains close to the optimum for the preparation of Metal-N-C SMAC, due its large cavity size of 11.8 angströms ( $\AA$ ). The synthesis of a Fe-N-C material from ZIF-8, featuring only atomically-dispersed Fe- $\mathrm{N}_{\mathrm{x}}$ sites, was demonstrated for the first time in 2015 [73]. The synthesis leaned on the drymixing of Fe acetate, phenanthroline and ZIF-8, avoiding any wet chemistry step that easily leads to Fe clustering by capillarity effects. Since then, other groups also achieved the complete atomic dispersion of the metal in Co- and Fe-N-C materials prepared from ZIF-8 under optimized conditions, with full atomic dispersion demonstrated using either ${ }^{57} \mathrm{Fe}$ Mössbauer spectroscopy, HR-STEM or XAS techniques $[66,68,74,75]$. Recently, two approaches relying on the soft-templating method were also investigated, that take advantage of chemical vapour deposition (CVD). In the first study, CVD was used to synthesize a Fe-doped ZIF, by reacting the gaseous ligand (2-methyl-imidazole) at $350{ }^{\circ} \mathrm{C}$ with $\mathrm{Fe}$-doped $\mathrm{ZnO}$ particles. This CVD-prepared catalyst precursor was then pyrolysed at $1000{ }^{\circ} \mathrm{C}$ to form a Fe-N-C SMAC [76]. In the second study, a Zn-N-C material with high density of $\mathrm{Zn}-\mathrm{N}_{4}$ sites was first prepared via pyrolysis of ZIF-8 and phenanthroline. $\mathrm{Zn}-\mathrm{N}_{4}$ sites were then used as platform for the formation of $\mathrm{Fe}-\mathrm{N}_{4}$ sites via $\mathrm{CVD}$ of $\mathrm{FeCl}_{3(\mathrm{~g})}$ at $750{ }^{\circ} \mathrm{C}$, leading to the high-temperature transmetalation between $\mathrm{Zn}$ and $\mathrm{Fe}$ in the $\mathrm{N}_{4}$ cavity, and to a highly ORR-active Fe-N-C SMAC with Fe- $\mathrm{N}_{\mathrm{x}}$ sites located preferentially on the surface [77]. A low-temperature transmetalation had previously been proposed by Fellinger's group, with a magnesium(Mg)-N-C material comprising $\mathrm{Mg}-\mathrm{N}_{\mathrm{x}}$ sites [78], but the resulting Fe-N-C had however moderate ORR activity.

\subsection{Sacrificial polymers}

Nitrogen-doped polymers have been studied as a suitable precursor of nitrogen and carbon, with the nitrogen-groups being also useful to bind metal cations and disperse them atomically before pyrolysis. Polyaniline and polypyrrole are perhaps the two most prominent examples of polymers that have been investigated for preparing Fe-N-C or other Metal-N-C SMAC, as reviewed in Ref. [79]. Wood et al. reported in 2007 on a highly active Co-N-C catalyst prepared from the polymerization of nitroaniline monomers in the presence of Fe chloride, followed by pyrolysis (illustrated in Figure 1c). Polyaniline can bind Fe cations either through the imine or amine groups, helping the Fe dispersion [80]. Since 2008, Zelenay's group has been highly active in 
research on Fe-N-C materials prepared via the sacrificial polymer approach [12,81-83]. Polyaniline has often resulted in the best performing Fe-N-C catalysts in their work, but the addition of some carbon support or even titanium oxide support during the polymerization step was however found useful to optimize the pore size distribution and electrocatalytic performance of the resulting catalysts. The aniline polymerization step was accelerated by the addition of an oxidant, such as ammonium peroxydisulfate. In this case, the introduced sulphur can also react during pyrolysis with excess $\mathrm{Fe}$ that cannot be hosted as $\mathrm{Fe}-\mathrm{N}_{\mathrm{x}}$ sites. Iron sulphur is then easily leached away during a subsequent acid-wash step. In 2017, Zelenay's group reported on an optimized synthesis involving polyaniline (PANI), cyanamide, BP2000 as a carbon black support and $\mathrm{FeCl}_{3}$, leading to a Fe-N-C catalyst containing only or mostly $\mathrm{Fe}-\mathrm{N}_{\mathrm{x}}$ sites, as evidenced by HR-STEM [84]. Cyanamide acted mainly as a pore-forming agent, and it was shown that much lower SSA was achieved for a similar synthesis but without cyanamide. HR-STEM evidenced the presence of $\mathrm{Fe}-\mathrm{N}_{\mathrm{x}}$ single sites, with a particularly high concentration at or near the edges of graphene sheets. The optimized catalyst resulted in a high PEMFC performance of $c a$ 0.9 and $0.39 \mathrm{~W} \mathrm{~cm}^{-2}$ geo in $\mathrm{H}_{2} / \mathrm{O}_{2}$ and $\mathrm{H}_{2} /$ air conditions, respectively. A recent work showed that designing PANI-like polymers having a stronger interaction with Fe cations could lead to higher amount of $\mathrm{Fe}$ adsorbed before pyrolysis, and higher density of $\mathrm{Fe}-\mathrm{N}_{\mathrm{x}}$ sites after pyrolysis [85]. Polymers with different nitrogen-bearing side chains were prepared, in order to increase the basicity of nitrogen-groups relative to PANI. With these polymers, the Fe content in the final catalysts could be increased by a factor of $c a 4$ compared to the Fe-N-C prepared from PANI, indicating that more Fe was converted to $\mathrm{Fe}-\mathrm{N}_{\mathrm{x}}$ sites. This increase was also reflected by increased ORR activity ( $c a \times 2.5$ at $E=0.8 \mathrm{~V} v s$. the reversible hydrogen electrode, RHE) and performance in PEMFC ( $+30 \%$ peak power density). This shows that, generally, the basicity of the nitrogen functional group in the starting polymers must be optimized.

\subsection{Electrospun polymer/MOF composites}

Another type of synthesis approach combining the sacrificial polymer approach and the sacrificial MOF approach was reported by Liu's group in 2015 [86]. It leaned on the electrospinning technique to prepare matts of metal-doped polymer/nano-MOF composite fibres, followed by their pyrolysis [86]. In this approach, the Fe-N-C phase derived from the pyrolysis of the nanoMOFs is the highly-active phase, and the integration of such nano-scaled domains in carbon 
fibres (derived from the pyrolysis of the polymer fibres) allows preparing hierarchically-structured catalyst with high amount of micropores (for hosting Fe- $\mathrm{N}_{\mathrm{x}}$ sites) and high volume of macropores (for high mass-transport properties). With such an approach, it is critical that the diameter of the MOF crystals be much smaller than the diameter of the polymer fibres formed during electrospinning, otherwise the MOF domains cannot be integrated inside the composite fibres. The ORR activity and PEMFC performance obtained with such advanced synthesis has been high, but not significantly higher than those obtained with less complicated separate polymer or MOF approaches, thus far. The electrospinning approach can also provide a pathway for the preparation of self-standing Fe-N-C electrodes, when the pyrolysis of the Fe, nitrogendoped polymer fibre matt retains mechanical integrity after pyrolysis. However, due to the void volume between fibres with electrospinning, the macropore volume in such self-standing electrodes is usually exceedingly high. This leads to overly thick and porous cathode layer for a given $\mathrm{Fe}-\mathrm{N}-\mathrm{C}$ loading, which is beneficial for $\mathrm{O}_{2}$ transport in the gas phase, but detrimental for the transport of protons.

\subsection{Synthesis of Metal-N-C SMAC beyond Fe and Co}

Without or with only a minor modification in the synthesis, it is most often possible to prepare Mn-, Ni-, $\mathrm{Cu}-$ and Zn-N-C SMAC with similar Metal-N $\mathrm{N}_{\mathrm{x}}$ coordination as found in Fe- and CoN-C SMAC $[32,87,88]$. Ni- and Cu-N-C SMAC typically have low ORR activity (comparable to $\mathrm{N}-\mathrm{C}$ ) but have been shown to have high selectivity and activity for $\mathrm{CO}_{2} \mathrm{RR}$, as reported from 2015 on [89] (see section 13.4.2). A Cr-N-C SMAC was reported in 2019, with promising ORR activity in acid medium [90]. One important application issue is the catalysis of Fenton reactions between in situ formed hydrogen peroxide $\left(\mathrm{H}_{2} \mathrm{O}_{2}\right)$ during ORR (especially, in acidic medium of PEMFCs) and 3d transition metals, leading to autocatalytic degradation or degradation of other components in the fuel cell, such as the membrane, or the ionomer in the cathode. From this perspective, $\mathrm{Fe}$ is regarded (perhaps misleadingly) as the worst choice of $3 \mathrm{~d}$ metal. The replacement of $\mathrm{Fe}$ by $\mathrm{Co}, \mathrm{Mn}$ or $\mathrm{Cr}$ in Metal- $\mathrm{N}_{\mathrm{x}}$ sites might therefore be appealing for improved operando stability at the cathode of PEMFC. However, all 3d transition metals have some Fenton reactivity, and among Metal-N-C materials, Fe is the most active for ORR, due to the highest TOF of Fe-N 4 sites. In 2020, the first highly active Metal-N-C material based on a $p$-block metal, namely $\mathrm{Sn}$, was reported, with $\mathrm{Sn}-\mathrm{N}_{\mathrm{x}}$ sites having similar TOF for ORR in acid medium 
than $\mathrm{Fe}-\mathrm{N}_{\mathrm{x}}$ sites [91]. However, the material contained also $\mathrm{SnO}_{\mathrm{x}}$ nanoclusters, in addition to Sn- $\mathrm{N}_{\mathrm{x}}$ sites, showing the challenge of selectively forming $\mathrm{Sn}-\mathrm{N}_{\mathrm{x}}$ sites. Interestingly, the latter have high catalytic activity and selectivity towards $\mathrm{CO}_{2}$ reduction, leading to a different $\mathrm{CO}_{2} \mathrm{RR}$ product than with Ni-N-C and Fe-N-C, as discussed in section 13.4.2. Zn- $\mathrm{N}_{\mathrm{x}}$ sites in $\mathrm{Zn}-\mathrm{N}-\mathrm{C}$ have generally no or low reactivity, but Zn-N-C SMAC with high density of $\mathrm{Zn}-\mathrm{N}_{\mathrm{x}}$ sites can be utilized as a platform for preparing, for example, Fe-N-C SMAC with a high density of sites as discussed earlier. The synthesis of Metal-N-C SMAC involving PGM has also been reported recently with various synthetic routes but that are similar to those established previously for $\mathrm{Fe}-$ $\mathrm{N}-\mathrm{C}$, for example for Pt [92], rhodium [93]. Pt-N $\mathrm{N}_{\mathrm{x}}$ sites in Pt-N-C have been found to efficiently catalyse the HER, with the advantage of increased utilization of expensive PGM, compared to PGM NP, where less than $100 \%$ of the metal is on the surface. In some studies, Pt SMA were prepared on nitrogen-doped carbon supports by simple adsorption, without pyrolysis, and high HER activity was also observed [94,95]. The nitrogen doping of the carbon was shown to be the key for retaining the atomic dispersion in operando conditions, with a reference Pt SMA on a nitrogen-free carbon leading to clusters and NP formation during HER [94]. Thus, depending on the strength of the Metal-N bond (in turn possibly related to the radius of the metal cations, since the $\mathrm{N}_{4}$ cavity in the carbon matrix must have a certain size, with limited flexibility) and the electrochemical operating conditions, subjecting the metal to a high temperature treatment is not always necessary for imparting activity and stability to SMA. Higher HER activity after high temperature treatment was however reported for Pt-N-C in Ref. [92]

\subsection{Synthesis of Metal-S-C SMAC}

While nitrogen is perhaps the strongest ligand for metal cations, other light elements can be targeted to stabilize SMAs in conductive carbon matrices, such as S, P, O. Several recent reports have demonstrated the pyrolytic synthesis of Metal-S-C materials, with Metal-S $\mathrm{S}_{\mathrm{x}}$ sites. For example, Choi et al. reported in 2016 on the synthesis of a Pt-S-C material, comprising ca $5 \mathrm{wt}$ \% of Pt atoms, all being present as Pt-S4 SMA site [96]. A S-doped carbon was first prepared with a hard-template method, resorting to a zeolite and depositing carbon on it via a CVD approach in acetylene/hydrogen sulphide at $550{ }^{\circ} \mathrm{C}$. The S-C material, featuring $17 \mathrm{wt} . \% \mathrm{~S}$, was then wet impregnated with a Pt salt, and reduced in $\mathrm{H}_{2}$ at $250{ }^{\circ} \mathrm{C}(\mathrm{Pt}-\mathrm{S}-\mathrm{C})$. No Pt NP were observed in Pt-S-C, but atomically-dispersed $\mathrm{Pt}$, coordinated by $4 \mathrm{~S}$ atoms according to extended $\mathrm{X}$-ray 
absorption fine structure (EXAFS) analysis. The Pt-S-C material catalysed the ORR to selectively form $\mathrm{H}_{2} \mathrm{O}_{2}(96 \%)$ in stark contrast to metallic Pt that mainly catalyses the ORR to form water (see more discussion on two-electron ORR electrocatalysis by carbon-embedded SMAC in section 13.4.1). Another Pt-S-C material with Pt-S4 sites was reported in 2019 [97], prepared via silica hard templating method but in an otherwise similar approach than in Ref [96]. Increasing the annealing temperature to $700{ }^{\circ} \mathrm{C}$ resulted in the conversion of $\mathrm{Pt}-\mathrm{S} 4$ sites into $\mathrm{Pt}$ clusters of $c a 1.5 \mathrm{~nm}$, and resulted in markedly different HER mass activity at an overpotential of $20 \mathrm{mV} v s$. RHE in $0.5 \mathrm{M} \mathrm{H}_{2} \mathrm{SO}_{4}$.

\section{Characterization methods and structure}

\subsection{Structure of SMA sites}

The structure of SMA sites in the Metal-N-C electrocatalysts bear similarities with those in the Metal-N $\mathrm{N}_{4}$ macrocyclic complexes, as discussed in Section 13.2, and the main trends between the nature of the metal cation and the activity of Metal-N-C SMAC for key electrochemical reactions, such as the ORR (see Section 13.4), are also similar to those observed with unpyrolysed metal macrocycles. The pyrolytic processes explored to synthesize the Metal-N-Cs aimed to design electrocatalysts with higher stability and activity than their molecular counterparts, consisting of a graphitic structure doped with Metal- $\mathrm{N}_{\mathrm{x}}$ moieties and, usually, also doped with additional nitrogen atoms that are not involved in metal ligation. In this section, we review the structural properties of the Metal-N-C electrocatalysts from atomic to macroscopic scale, and, specifically: (i) the nature of the different moieties, namely the Metal-N or nitrogen functionalities observed within the carbon structure and (ii) the carbon structural disorder and its effect on the electron density near the atomically dispersed metal. The pyrolysis leading to the formation of the Metal-N-C electrocatalysts incidentally generates a wide variety of different moieties, which can be divided in two main categories: (i) the metal-free moieties and (ii) the metalcontaining moieties. Metallic or other metal-rich NP are also observed in electrocatalysts synthesized with a high metal loading or with unoptimized synthesis approach. They encompass a wide range of carbides, nitrides, and metallic phases, which are often detrimental for the electrochemical performances and stability for the ORR [73,81], especially in acidic environment $[98,99]$, or detrimental to the $\mathrm{CO}_{2} \mathrm{RR}$ selectivity [100]. The metal-free moieties consist in diverse nitrogen-based structures, namely N-pyridinic, N-pyrrolic, N-graphitic, N-protonated, 
etc. [101] whose exact contribution to the electrocatalytic activity of the Metal-N-Cs remains highly debated, and probably depends on the $\mathrm{pH}$ (for ORR), and on the relative amount of $\mathrm{N}$ moieties and Metal- $\mathrm{N}_{\mathrm{x}}$ moieties in each Metal-N-C material.

It is widely accepted, however, that the metal-based moieties are often the main contributor to the Metal-N-C's performances, in the case of optimized materials with relatively high amount of Metal-N $\mathrm{N}$ SMA sites. As such, they are the main focus of this discussion. More specifically, the discussion is to focus on Fe- $\mathrm{N}_{\mathrm{x}}$ moieties, owing to the fact that ${ }^{57} \mathrm{Fe}$ Mössbauer spectroscopy has been applied extensively to Fe-N-C materials ex situ, and even in situ or operando recently. This technique is particularly well suited to identify the oxidation state and spin state of atomically dispersed Fe, and combined with XAS or HR-STEM, makes the understanding of the FeN-C active sites structure more advanced than for other Metal-N-C SMAC for which Mössbauer spectroscopy is typically not achievable or not easily accessible. However, other Metal$\mathrm{N}-\mathrm{C}$ were studied and some of the structural conclusions discussed in this section can be extended to them (e.g. Co-N-C, Sn-N-C, etc.). For example, Co-N-C SMAC have been studied instead with XAS, resorting to the calculation of X-ray absorption near-edge spectroscopy (XANES) spectra for a library of Co- $\mathrm{N}_{\mathrm{x}}$ sites, and comparing to the experimental XANES spectrum collected on all Co atoms in the material [102].

\subsubsection{Different $\mathrm{Fe}-\mathrm{N}_{\mathrm{x}} \mathrm{C}_{\mathrm{y}}$ moieties}

For $\mathrm{Fe}-\mathrm{N}-\mathrm{C}$, the $\mathrm{Fe}-\mathrm{N}_{\mathrm{x}}$ moieties have been hypothesized to exist under various forms, e.g. (i) Fe-N 4 [103] (illustrated in Figure 1a), (ii) Fe- $\mathrm{N}_{2+2}$ [104] and (iii) $\mathrm{N}-\mathrm{Fe}-\mathrm{N}_{2+2}$ [104]. Furthermore, the Fe coordination is believed to change with its position in the carbon basal plane, leading to the presence of $\mathrm{Fe}-\mathrm{N}_{2}$ (on the carbon edge), $\mathrm{Fe}-\mathrm{N}_{3}$ [105] or generically $\mathrm{Fe}-\mathrm{N}_{\mathrm{x}} \mathrm{C}_{\mathrm{y}}$ sites (e.g. $\mathrm{Fe}$ coordinated to $x$ nitrogen atoms in the first coordination sphere, and $y$ carbon atoms in the second coordination sphere). N-Fe- $\mathrm{N}_{2+2}$ and $\mathrm{Fe}-\mathrm{N}_{2+2}$ are often assumed to be found as 'bridges' between two graphite crystallites defining micropores (pore size $<2 \mathrm{~nm}$ ) [104,106], whereas the Fe- $\mathrm{N}_{4}$ exist in carbon basal planes and, thus, likely, in mesopores $(2 \mathrm{~nm}<$ mesopores $<50$ $\mathrm{nm})$. Their different coordination with nitrogen directly impacts their electronic structure and the spin state of Fe. For example, it was discussed that $\mathrm{Fe}-\mathrm{N}_{\mathrm{x}}$ moieties with different orbital filling can present either an empty $d_{\mathrm{z} 2}$, a filled $d_{\mathrm{z} 2}$ or a half-filled $d_{\mathrm{z} 2}$ orbital [106]. This impacts the binding strength and conformation of various reactive intermediates on their surface. With 
a filled $d_{\mathrm{z} 2}$ orbital, the Fe cation in $\mathrm{Fe}-\mathrm{N}_{\mathrm{x}}$ cannot bind with oxygen in a non-bent configuration, hence implying a weaker binding than Fe- $\mathrm{N}_{\mathrm{x}}$ site with emptied $d_{\mathrm{z} 2}$ orbital, able to form an $\sigma$ bond with $\mathrm{O}_{2}$. When addressing the $\mathrm{Fe}-\mathrm{N}_{\mathrm{x}}$ sites, their local coordination, and therefore the nature (pyridinic vs. pyrrolic) of the nitrogen atoms bound to $\mathrm{Fe}$, was experimentally investigated through the comparison of calculated and measured XANES spectra, for a Fe-N-C material that was independently demonstrated by EXAFS and Mössbauer to contain no Fe clusters. The coordination was evidenced to be mainly a near-planar Fe-N4- $\mathrm{C}_{12}$ on a Fe-N-C synthesized from ZIF-8, i.e. an Fe coordinated with four N-pyrrolic [73].

Here, it is important to note that the label of $(\mathrm{N}-) \mathrm{Fe}-\mathrm{N}_{2+2}$ moieties was previously proposed and used to reflect the fact that these sites are hosted in micropores, and therefore probably involving carbon atoms from two different graphitic crystallites. This is as opposed to in-plane Fe- $\mathrm{N}_{4}$ site, with all carbon atoms belonging to the same crystallite. However, by introducing structural disorder in a graphene sheet (including five-carbon atom cycles), it is also possible to produce a variety of $\mathrm{Fe}-\mathrm{N}_{\mathrm{x}}$ moieties, from the "perfect defect" corresponding to two-carbon defects (for creating space for the metal cation) in a perfect graphene sheet and the replacement of four carbon atoms by nitrogen atoms (labelled usually as $\mathrm{FeN}_{4} \mathrm{C}_{10}$ site), to a highly defective graphene sheets in which a $\mathrm{FeN}_{4} \mathrm{C}_{12}$ site can be created, with structure of its two first coordination spheres very similar to that existing in Fe porphyrin. We also highlight that the terminology of pyrrolic and pyridinic nitrogen in the field of Metal-N-C is not free of ambiguity. Strictly speaking, this is derived from the chemical state of nitrogen in pyridine and in pyrrole molecules. In the field of Metal-N-C, the terminology of pyridinic and pyrrolic is usually referred to on the basis of X-ray photoelectron spectroscopy (XPS) N1s deconvolution. Although, originally, the binding energies of said molecules were used for deconvolution of the Metal-N-C N1s envelope, recent works have been highlighting the need to consider a binding energy shift when assessing the N-containing moieties by XPS [107,108]. This 'core-level-shift' is especially important when discussing the Metal-N bond, and leads to shifts in binding energy $>1 \mathrm{eV}$, but also impacts the binding energy of nitrogen depending on its position in the carbon basal plane, e.g. edge vs. plan. In the more recent XANES work applied to Fe-N-C, however, the terminology of pyrrolic or pyridinic is more related to the way the nitrogen atoms are embedded in the carbon matrix (as this is the information provided by XANES by Zitolo et al. [73]), and in 
particular, if it is involved in six or five-membered rings of light elements. In this context, the definition of pyrrolic/pyridinic is therefore geometrical, and not chemical, by nature.

\subsubsection{Macroscopic structure}

As a result of its embedded nature in the basal plane or on the edges of graphitic crystallites, the reactivity of Fe- $\mathrm{N}_{\mathrm{x}}$ moieties is not only related to the local structure in the first 1-3 coordination spheres, but also highly dependent of the long range carbon neighbours, including the graphitization degree of the carbon matrix, the presence of structural defects (e.g. missing atoms, nitrogen-containing moieties) but also its acido-basicity and electron delocalization. Some of these long-range parameters of a carbon material surface are intertwined (see section 13.5.4). The overall morphology of a Metal-N-C catalyst, i.e. size of graphitic crystallites, micro/mesoporous pore size distribution, functionalization with nitrogen and/or oxygen (oxygen is always present on surface, after exposure to air), depends on the synthesis procedure, as detailed in Section 13.2. For example, a Metal-N-C synthesized from a hard-templated method, using silica spheres $>20 \mathrm{~nm}$, will certainly result in mesoporous or micro-mesoporous Metal-N-C. In contrast, a Metal-N-C synthesized from a MOF by sacrificial approach may show only/mostly micropores [59]. However, the materials that comprise only or mostly Metal- $\mathrm{N}_{\mathrm{x}}$ sites (no Metal particles) are always carbon-rich and therefore share morphological properties similar to other carbon supports, i.e. several levels of porous networks (micro-, meso-, macroporous) that together lead to the formation of a hierarchical structure. The variations in porosities and their distribution can impact the proportion of different Fe- $\mathrm{N}_{\mathrm{x}}$ actives sites, i.e. the soft-templated Fe-N-C materials (from ZIF-8) are believed to exhibit an enhanced presence of microporehosted sites $v s$. hard-templated Fe-N-C electrocatalysts. A clear interconnection between the type of Fe- $\mathrm{N}_{\mathrm{x}}$ sites and the carbon matrix morphology is however not yet established. Developing methods that can differentiate a basal graphite surface from surfaces created by the edges in complex carbon materials could help progressing on this topic. In addition, $\mathrm{N}_{2}$ sorption isotherms, while efficient at capturing the micro and mesopores, are not suited to assess the macropores in catalyst powders and in catalytic layers. Macropores are often the result of the electrode preparation method, and ink drying process, and key to gas-phase transport in catalytic layers. 


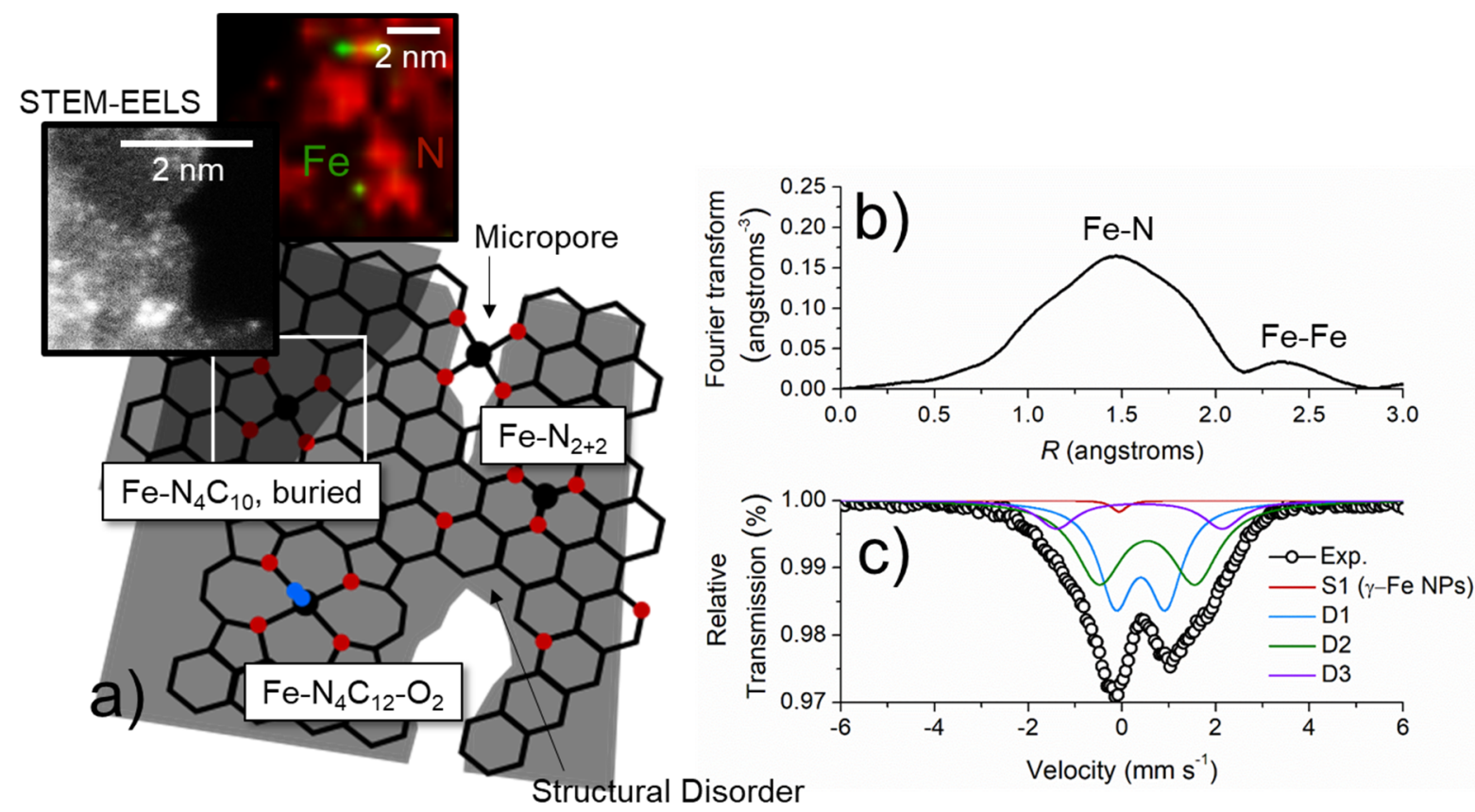

Figure 2. Illustration of the Fe-moieties local coordination and of the experimental tools used to assess their existence. a) graphical representation of a disordered graphene plane, with an illustration of the various metal-containing moieties observed in metal-nitrogen-carbon electrocatalyst and a micrograph of iron atoms embedded in the carbon structure along with an elemental map distribution (the micrograph and the elemental map do not correspond to the same region); b) Fourier transform of the extended X-ray absorption fine structure obtained for an Fe-N-C electrocatalyst, with the main features and c) Mössbauer experimental data and fitting with a singlet component (corresponding to $\gamma$-Fe particles) and three doublets corresponding to different Fe- $\mathrm{N}_{\mathrm{x}}$ SMA sites. Source: Tristan Asset, Frédéric Maillard, Frédéric Jaouen.

It is believed that the oxidation degree, and the out-of $v s$. in-plane configuration of the Fe- $\mathrm{N}_{4}$ moieties impact their reactivity, whether it is for the $\mathrm{CO}_{2} \mathrm{RR}$ to $\mathrm{CH}_{4}$, with the $\mathrm{Fe}(\mathrm{II}) \rightarrow \mathrm{Fe}(\mathrm{I})$ hypothetical transition at very low potential, or the ORR, with the out-of-plane $\mathrm{Fe}(\mathrm{II}) \rightarrow$ inplane $\mathrm{Fe}(\mathrm{III})$ transition $[109,110])$. The potential at which the $\mathrm{Fe}(\mathrm{III}) / \mathrm{Fe}(\mathrm{II})$ transition occurs for the ORR is correlated to how delocalized the $\pi$-electron are in the carbon basal plane, which in turn depends on the carbon structural disorder [111].

\subsubsection{Importance of the carbon surface and $\pi$-electron delocalization}


The structural order of the carbon matrix takes various aspects, which impact the $\pi$-electron delocalization on two levels (i) the crystallite size, i.e. smaller crystallites, and thus more disordered $\mathrm{C}$, result in a disruption of the $\pi$-electron delocalization, similarly to (ii) the presence of heteroatoms (e.g. nitrogen-groups, $\mathrm{Fe}-\mathrm{N}_{\mathrm{x}}$, or oxygen-groups) and other structural defects (holes, edges-sites, etc.), as illustrated in Figure 2a. A disruption of the $\pi$-electron delocalization increases the electron withdrawing nature of the graphene plane, thus affecting the electron density at Fe- $\mathrm{N}_{\mathrm{x}}$ sites and their TOF and selectivity. Ramaswamy et al. showed that the initial ORR activity of Fe-N-C catalysts prepared from the pyrolysis of a Fe-porphyrin and vastly different carbon black supports (from low to high surface area, and from highly ordered to highly disordered) could be interpreted in terms of modified $\pi$-electron delocalization around Fe-N $\mathrm{N}_{\mathrm{x}}$ sites [111]. Choi et al. showed, with a single Fe-N-C catalyst, that introducing numerous oxygen functional groups on the carbon surface with different $\mathrm{H}_{2} \mathrm{O}_{2}$ treatments resulted in an ORR activity decrease that correlated with the increased acidity of the surface and increased work function, i.e. decreased $\pi$-electron delocalization (see also section 13.5.4) [112].

\subsection{Characterization methods dedicated to Metal-N-C SMAC}

One of the key challenges encountered when working with SMAC is that traditional physicochemical characterization techniques such as X-ray diffraction (XRD), scanning electron microscopy combined with energy dispersive X-ray spectroscopy (SEM/X-EDS), accessible in many laboratories, do not have sufficient precision/resolution to distinguish if atoms are dispersed in the atomic state or not. In fact, only a certain number of selected characterization techniques are suited to unveil the presence of SMA sites. In what follows, we restrict ourselves to these must-have characterization techniques, describe their principle, the information they allow to reach and discuss representative papers, which used them. The most straightforward technique to examine SMAC with a resolution of the order of the $\AA$ is scanning transmission electron microscopy (STEM). In this technique, an ultra-small electron probe is swept over the sample, and the transmitted electrons are collected by a high angle annular dark field detector (HAADF). Correction of spherical and chromatic aberrations (aberration-corrected STEM, ACSTEM) significantly improves the resolution of traditional STEMs, providing sub-Å resolution well adapted to SMAC. Combination with electron energy-loss spectroscopy (EELS) provides additional insights into chemical environment. For example, STEM-EELS allows visualizing 
how transition metal atoms are distributed in the carbon matrix (either as SMA or under the form or metal/metal carbides/metal oxides NP), and distinguishing whether nitrogen atoms are located in their vicinity [113]. This is illustrated in Figure 2a, showing single Fe atoms dispersed on a carbon matrix and demonstrating the presence of nitrogen atoms in their vicinity.

Complementary to AC-STEM, XAS is an element-specific characterization technique providing insights into the oxidation state of SMAC and into the coordination of the transition metal element $[73,84,102,114]$. While the XAS technique is only accessible in synchrotron facilities (except for the recent bench-top equipment, but not suited to look at dilute samples such as Metal-N-C), the developed EXAFS analysis is easily accessible and allows to routinely demonstrate if a SMAC has been synthesized, or if a Metal-N-C SMAC comprises of mix of SMAs and particles, or mostly metal particles. Practically speaking, SMAC powder or a pellet of powder is illuminated with a monochromatic beam of X-ray photons either ex situ [73,115] in situ $[116,117]$ or operando $[102,118]$. Absorption of X-rays by the transition metal atoms leads to the ejection of photoelectrons, and formation of excited core-hole states. For X-ray photons energies near the absorption edge ( $E_{0}$, referred to as the XANES region), backscattering of the excited photoelectrons by the surrounding atoms provides information on the oxidation state, the orbital occupancy and the local coordination of the transition metal atoms. For energies well above the absorption edge (i.e. starting at $E_{0}+100 \mathrm{eV}$ ), the EXAFS region is a fingerprint of the nature and number of atoms present in the first coordination spheres (i.e. from 1 to 5-6 $\AA$, see Figure 2b). Typically, Fourier-Transform EXAFS (FT-EXAFS) can identify a peak at $c a$ $1.5 \AA$, related to Metal-N coordination, while peaks due to $\mathrm{Fe}-\mathrm{Fe}$ backscattering can be expected at $c a 1.2$ and $4.5 \AA$, if metallic Fe particles are present (similar atomic distances are assessed for different metal atoms such as Co, Sn, Mn. We detail here the case of Fe-N-C for the sake of clarity). Fitting of the FT-EXAFS of pure SMAC can provide more information, such as exact distance, and the average coordination number of Fe with its first neighbours, etc. While in the case of SMAC free of metal clusters, the FT-EXAFS analysis and output is relatively facile to understand, for SMAC containing even a small fraction of metal clusters, the output becomes quickly complex. This is because the EXAFS signal is averaged on all Fe atoms present, and in the case of mix Fe-N $\mathrm{N}_{\mathrm{x}}$ sites and Fe particles for example, the coordination number found by fitting for Fe-N, for example (and same for Fe-Fe) loses its physical meaning at the single-site level. For example, a theoretical mix of $50 \% \mathrm{Fe}$ as $\mathrm{Fe}-\mathrm{N}_{4}$ sites and $50 \% \mathrm{Fe}$ as $\mathrm{Fe}$ clusters 
would result in a Fe-N apparent average coordination number by FT-EXAFS of 2.0, even though there is no single site $\mathrm{Fe}-\mathrm{N}_{2}$ present in the material. Therefore, detailed structural characterisation of SMA sites in SMAC is preferably done in the absence of any metal clusters.

Complementary to experimental EXAFS and XANES as well as calculated EXAFS (leading to the knowledge of the type, number and bond distance between the absorber $(\mathrm{Fe})$ and surrounding atoms), the recent development of methods to calculate XANES spectra from optimized density functional theory (DFT) structures and to compare them to experimental spectra has provided further information on the site geometry of SMAs, since XANES is more sensitive than EXAFS to the spatial distribution of surrounding atoms. The combined application of calculated XANES spectra for optimized hypothetical Fe- $\mathrm{N}_{\mathrm{x}}$ structures and comparison to XANES experimental spectrum of a model Fe-N-C material free of Fe clusters leading to pioneering advances as the work of Zitolo et al. evidenced the porphyrinic planar architecture with a $\mathrm{FeN}_{4} \mathrm{C}_{12}$ core and with $\mathrm{O}_{2}$ adsorbed in the side-on or end-on configuration of metal-containing moieties observed in Fe-N-C, as discussed previously $[73,102,108]$. Complementary to XAS is $\mathrm{X}$-ray emission spectroscopy (XES). This technique is based on the measurement of fluorescence photons emitted upon transfer of electrons from an outer-shell to the electron hole, and renders changes in the oxidation state and spin state observable. Saveleva et al. reported recently that XES is capable of tracking in situ the changes of oxidation state and spin state of single Fe atoms in ORR conditions [119].

With some similarity to the XANES principle, ${ }^{57} \mathrm{Fe}$ Mössbauer spectroscopy is based on the recoilless absorption of $\gamma$-rays by ${ }^{57} \mathrm{Fe}$ atoms. It does not require any crystalline order of the $\mathrm{Fe}$ atoms, and is therefore well suited for Fe-N-C SMAC [120]. The technique is extremely sensitive to tiny changes in the energy levels of the Fe nucleus, implied by the local environment around each Fe atom, changing the oxidation state, spin state and the electric field gradient around Fe. In addition, ${ }^{57} \mathrm{Fe}$ Mössbauer spectroscopy is powerful at distinguishing crystalline phases of $\mathrm{Fe}$ from $\mathrm{Fe}$ single-atom sites, since most Fe crystalline phases in $\mathrm{Fe}-\mathrm{N}-\mathrm{C}$ materials have magnetic properties, resulting in sextet signals in the Mössbauer spectra (e.g. Fe carbides, Fe oxides and $\alpha-\mathrm{Fe}$ ). In contrast, Fe-based SMA sites have no magnetic properties due to their dispersed nature, resulting in a doublet signature in Mössbauer spectra. This key difference allows an easy quantification of SMA sites and Fe clusters, for most Fe-N-C materials, by fitting 
the Mössbauer spectra with a set of doublets and sextets. In addition, a singlet component for the $\gamma$-Fe metallic phase is sometimes necessary. This metastable phase of metallic Fe is often found in Fe-N-C catalysts comprising Fe clusters, due to the rapid quenching from high temperature (where $\gamma$-Fe is more stable than $\alpha-\mathrm{Fe}$ ) at the end of pyrolysis.

In the field of Metal-N-C materials, Mössbauer spectroscopy has most often been used to study monometallic Fe-N-C catalysts [20,38,73,120], as illustrated in Figure 2c, but has recently been used for Sn-N-C catalysts comprising Sn atomically dispersed in a nitrogen-doped carbon matrix. Mössbauer spectra are on the first hand used to assess the relative amounts of Fe crystalline phases and of SMA sites. [102]. From ex situ Mössbauer spectroscopy on many Fe-N-C SMAC, two main doublets (most often labelled D1 and D2 in the literature) have been ubiquitously observed, differing mainly in their quadrupole splitting (QS, represented by the peak to peak "distance" of a given doublet). These doublets have initially been interpreted by comparison to doublets experimentally measured for unpyrolysed $\mathrm{FeN}_{4}$ macrocycles with known oxidation and spin states. Recently, DFT methods have been developed to calculate the QS and the isomer shift (IS) of different Fe- $\mathrm{N}_{\mathrm{x}}$ hypothetical sites embedded in (disordered) graphene sheets, which rationalizes the interpretation of Mössbauer spectra of pyrolysed Fe-N-C SMAC [20,121,122]. The DFT results nevertheless showed the ambiguity of the spectroscopic response. For example, three different sites (namely $\mathrm{Fe}(\mathrm{II}) \mathrm{N}_{4} \mathrm{C}_{12}$ site with spin state 0 or 1 but also $\mathrm{Fe}(\mathrm{III}) \mathrm{N}_{4} \mathrm{C}_{12}$ with spin state $5 / 2$ or $\mathrm{Fe}(\mathrm{III}) \mathrm{N}_{4} \mathrm{C}_{10}$ with spin $5 / 2$ ) all lead to QS-values around $1 \mathrm{~mm} \mathrm{~s}^{-1}$, compatible with the experimental D1 [20]. In contrast, only the QS-values calculated for the $\mathrm{Fe}(\mathrm{II}) \mathrm{N}_{4} \mathrm{C}_{10}$ (in spin state either 0 or 1 ) moiety matched the experimentally observed value of D2 (2.4-3.0 $\mathrm{mm} \mathrm{s}^{-1}$ ) [20]. Generally, Mössbauer spectroscopy must therefore be combined with other techniques probing the oxidation and/or spin state of Fe to safely conclude on the different doublets. While ex situ Mössbauer spectroscopy has been applied for a long time to Fe-N-C SMAC, in situ or post mortem investigations have been almost inexistent until 2019, due to the high dilution of $\mathrm{Fe}$ (typically, less than 2 wt. \% Fe as atomic site) and the low \% of the ${ }^{57} \mathrm{Fe}$ isotope (Mössbauer-active) in natural $\mathrm{Fe}$, making such experiments challenging, even with ${ }^{57} \mathrm{Fe}$ enriched $\mathrm{Fe}-\mathrm{N}-\mathrm{C}$.

In 2021, in situ Mössbauer spectroscopy in a PEMFC allowed revealing the potential-dependent nature of D1 (switching between Fe(III) and Fe(II) states) and potential-independent nature of 
D2 (remaining Fe(II)) [123]. Such measurements are however also challenged by the low stability of Fe-N-C materials in acidic medium, with a fraction of the D1 sites quickly converting to nanosized Fe-oxides. Due to their size, such oxides are superparamagnetic, and contribute with a doublet signal that is very similar to the true D1 signal. The nanosized Fe oxides can be separated from the true D1 signal only at low temperature (typically below $-253{ }^{\circ} \mathrm{C}$, i.e. far away from electrochemical conditions), since at such low temperature they contribute with a sextet signal, as large Fe-oxides do. This observation conducts us to mention the promising application of Mössbauer spectroscopy to characterise Fe-N-C electrodes after electrochemical operation in PEMFC or other devices, in which case the low-temperature measurement is critical to distinguish nano-Fe oxides from a true D1 signal related to $\mathrm{Fe}-\mathrm{N}_{\mathrm{x}}$ sites [123]. First reports have shown that the D2 signal is stable in operating PEMFC, while D1 quickly converts to nano-Fe oxides. In this frame, post mortem low-temperature Mössbauer spectroscopy proved much more efficient than XANES-EXAFS at quantifying the contribution of D2, D1 and nanoFe-oxides [123]. The reason is that nano-Fe-oxides have a XANES spectrum very similar to that of $\mathrm{Fe}-\mathrm{N}_{\mathrm{x}}$ sites, and the Fe-Fe signal from amorphous Fe-oxides is poorly visible in EXAFS.

Complementary to Mössbauer spectroscopy, nuclear inelastic scattering (NIS) consists of measuring the energy spectrum of inelastic absorption of X-rays, from a synchrotron beam, by nuclei. A signal of elastic nuclear absorption arises, when the energies of incident X-rays and nuclear transition coincide. Nuclear absorption can also occur inelastically with creation or annihilation of lattice vibrations. The latter processes cause inelastic sidebands in the energy spectra around the elastic peak, which lead to the partial density of phonon states. NIS provides information of the energy spectra of the recoil, i.e. the frequency distribution of the lattice vibrations and allows the identification of modes which are not available otherwise. By combining Mössbauer spectroscopy, NIS and DFT calculations, Kneebone et al. identified the Fe sites that are ORR active in a PANI-based Fe-N-C catalyst [124]. Wagner et al. recently used NIS to detect Fe oxide species on the surface of a Fe-N-C material [125].

Except for in situ Mössbauer spectroscopy, which can be surface-specific, but is challenging and definitely not a routine method, and in situ EXAFS-XANES, other techniques have been developed to probe the electrochemically active sites in Fe-N-C and other Metal-N-C SMAC. By definition, both Mössbauer spectroscopy and XAS are probing the bulk of the material, due 
to the high energy ( $c a 7 \mathrm{keV}$ for XAS at Fe $K$ edge and $14.4 \mathrm{keV}$ for ${ }^{57} \mathrm{Fe}$ Mössbauer). To tackle this shortcoming, cyclic voltammetry, a technique in which the electrode potential is swept linearly with time between two potential limits, is of prime interest. Kucernak's group [126] first reported an electrochemical method, based a sodium nitrite probe molecule $\left(\mathrm{NaNO}_{2}\right)$ that can be strongly adsorbed on SMA and electrochemically removed, via electroreduction, from the electrocatalyst surface thus allowing estimation of the density of SMAs located on the surface (ASD). Unfortunately, $\mathrm{NaNO}_{2}$ molecules indifferently probe single metal Fe atoms and Fe-oxides/Fe-carbide species [127]. An ex situ chemisorption/desorption-based method, using low temperature $\left(-100^{\circ} \mathrm{C}\right) \mathrm{CO}$ chemisorption [128], was recently reported by Strasser's group to determine the site density of SMAC based on Fe or Mn atoms. Low temperature was in this case necessary, since $\mathrm{CO}$ does not adsorb strongly on $\mathrm{Fe}-\mathrm{N}_{\mathrm{x}}$ or $\mathrm{Mn}-\mathrm{N}_{\mathrm{x}}$ sites at room temperature (explaining why such sites are tolerant to $\mathrm{CO}$ in electrochemical conditions, in contrast to $\mathrm{Pt}$ ). Another recent quantification method using a cyanide anion as a probe molecule was published by Choi's group [129]. The method proved valid to determine the active site density of various transition/noble metal SMAC beyond Fe and Mn, and also in a broad $\mathrm{pH}$ range (1-13).

\section{Applications in electrocatalysis}

\subsection{Oxygen reduction reaction}

The ORR has been the first electrochemical reaction investigated for the application of MetalN-C SMAC, and of Fe-N-C SMAC in particular. The ORR can lead to the formation of water

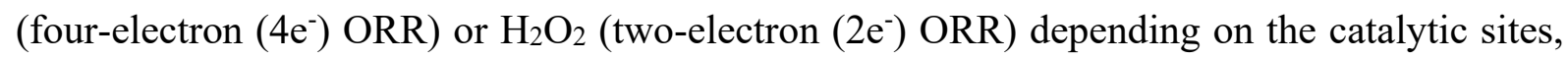
electrode morphology and experimental conditions. In fuel cells, water is the desired ORR product, not only in order to maximize the current density per flux of $\mathrm{O}_{2}$ diffusing inside the electrode, but also to prevent undesired reactions between $\mathrm{H}_{2} \mathrm{O}_{2}$ and the catalyst, ionomer or membrane materials. $\mathrm{H}_{2} \mathrm{O}_{2}$ is a strong oxidant, which can also chemically react with transition metals (in particular, $\mathrm{Fe}$ ), forming even more oxidizing reactive oxygen species, such as $\mathrm{OH}^{\bullet}$ radicals. In a fuel cell cathode, the presence of multitudinous active sites often leads to the co-existence of different paths for the ORR, including direct $4 \mathrm{e}^{-} \mathrm{ORR}, 2 \mathrm{e}^{-} \mathrm{ORR}$ (releasing desorbed $\mathrm{H}_{2} \mathrm{O}_{2}$ ), and indirect $4 \mathrm{e}^{-} \mathrm{ORR}$, implying the formation of $\mathrm{H}_{2} \mathrm{O}_{2}$ on one active site (first site), its desorption, and re-adsorption on another (second) site where $\mathrm{H}_{2} \mathrm{O}_{2}$ is electrochemically reduced to water. When the nature of the second active site is the same as that of the first site, the ORR 
pathway is described as being $2 \mathrm{e}^{-} \times 2 \mathrm{e}^{-}$, while when the first and second site differ in nature, the pathway is described as being $2 \mathrm{e}^{-}+2 \mathrm{e}^{-}[109]$ (see Figure 3a for an illustration of the pathways)

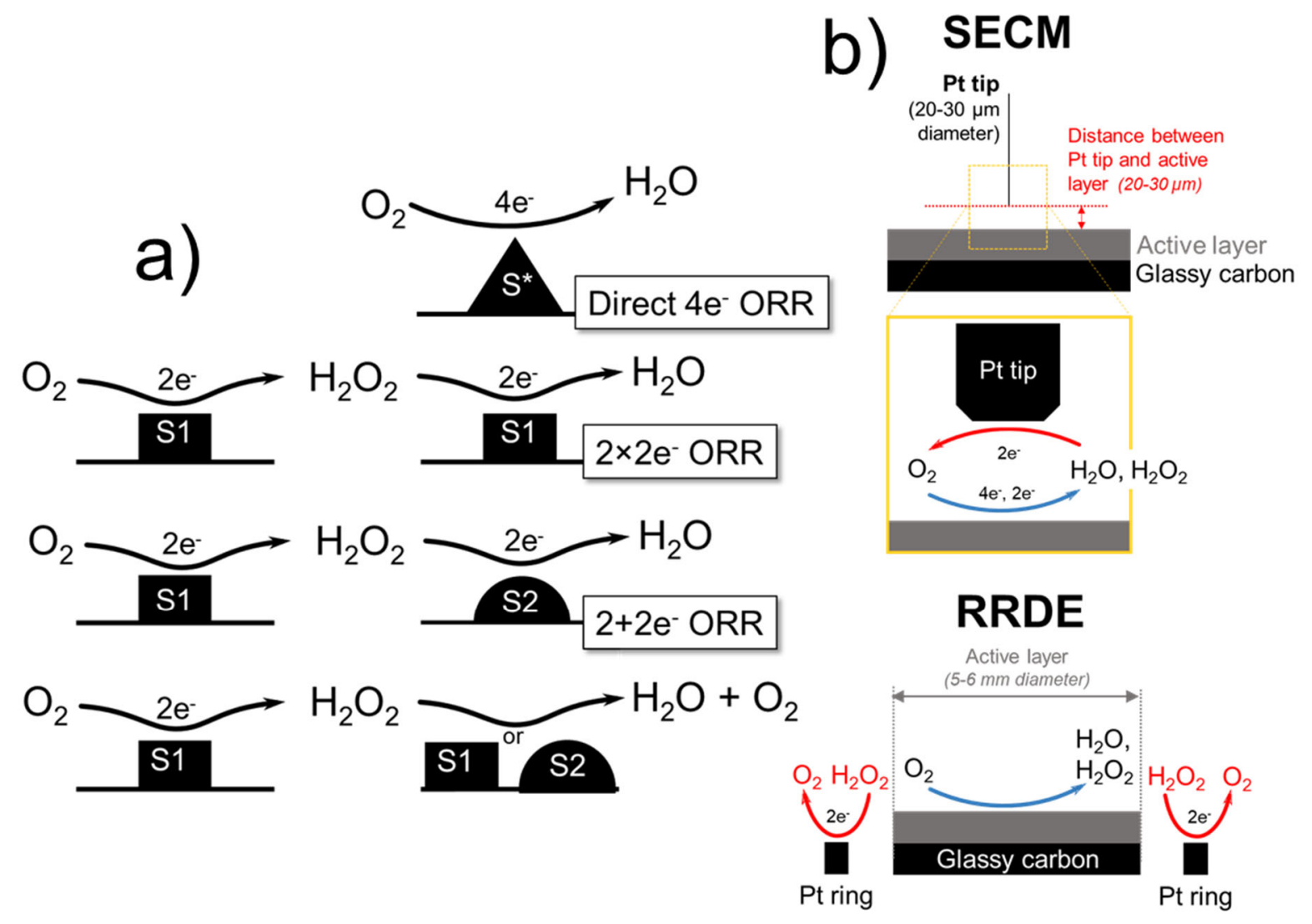

Figure 3. Illustration of the mechanistic and instrumental concepts involved in the study of the oxygen reduction reaction on metal-nitrogen-carbon electrocatalysts. a) Direct and indirect oxygen reduction reaction (ORR) mechanisms and b) scheme of scanning electrochemical microscopy (SECM) and rotating ring disk electrode (RRDE) techniques that can measure the selectivity of electrocatalysts during ORR. Source: Tristan Asset, Frédéric Maillard, Frédéric Jaouen.

In practice, identifying whether the ORR pathway on a given catalyst (always containing a high number of active sites) is $4 \mathrm{e}^{-}, 2 \mathrm{e}^{-}+2 \mathrm{e}^{-}$or $2 \mathrm{e}^{-} \times 2 \mathrm{e}^{-}$is very challenging. In addition, $2 \mathrm{e}-$ ORR followed by the non-electrochemical $\mathrm{H}_{2} \mathrm{O}_{2}$ disproportionation into $\mathrm{O}_{2}$ and $\mathrm{H}_{2} \mathrm{O}$, can, after several "cycles", lead to an apparent low production of $\mathrm{H}_{2} \mathrm{O}_{2}$ during ORR. Answering the question whether a given catalyst mainly catalyses the ORR through $4 \mathrm{e}^{-}, 2 \mathrm{e}^{-}+2 \mathrm{e}^{-}, 2 \mathrm{e}^{-} \times 2 \mathrm{e}^{-}$, or $2 \mathrm{e}^{-}+$ disproportionation reaction bears not only fundamental importance, but also practical ones. 
Even if a low apparent amount of peroxide is produced initially, the existence of desorbed "free" $\mathrm{H}_{2} \mathrm{O}_{2}$ inside the cathode with all indirect ORR mechanisms can lead to rapid degradation of performance over time. For example, Tylus et al. investigated the ORR pathway on three different Fe-N-C catalysts (with only SMA Fe-N $\mathrm{N}_{\mathrm{x}}$ sites, or a mix of Fe- $\mathrm{N}_{\mathrm{x}}$ sites and Fe particles), and both in acid and alkaline media [109]. They concluded that, in alkaline medium, Fe- $\mathrm{N}_{\mathrm{x}}$ sites can catalyse the direct $4 \mathrm{e}^{-}$ORR and the $2 \mathrm{e}^{-} \times 2 \mathrm{e}^{-}$indirect pathway, while in acid, the role of $\mathrm{Fe}$ particles (Fe-oxides, or metallic Fe or Fe carbide encapsulated in a carbon shell) in catalysing $\mathrm{H}_{2} \mathrm{O}_{2}$ electroreduction to water was invoked for explaining the rotating ring disk electrode (RRDE) experimental data. Other studies however reported low \% peroxide during ORR in acid media for Fe-N-C catalysts free of Fe particles (as measured ex situ [130]). In order to experimentally differentiate direct $4 \mathrm{e}^{-}$ORR from indirect ORR mechanisms, it is important to keep in mind that the catalytic layer should be made as thin as possible, in order to quickly remove from the layer any peroxide that is produced, in order to prevent further reactions and to assess the true ORR mechanism. While this effect is generally valid for all ORR catalysts, including Pt/C catalysts [131], the effect was shown to be particularly strong for a particular Fe-N-C catalyst for ORR in acid medium in 2008 [132], with the $\% \mathrm{H}_{2} \mathrm{O}_{2}$ measured by RRDE increasing from 5 to $95 \%$ when the loading was decreased from 800 to $50 \mu \mathrm{g} \mathrm{cm}^{-2}$ geo.

The role of the nature of the metal cation in defining the ORR selectivity of Metal- $\mathrm{N}_{\mathrm{x}}$ sites has been regularly investigated, first on Metal-N-C catalysts with undefined metal speciation, or comprising a mix of metal particles and Metal- $\mathrm{N}_{\mathrm{x}}$ sites. Recent works on Metal-N-C materials comprising only or mostly Metal- $\mathrm{N}_{\mathrm{x}}$ sites confirm the previous reported trends, with generally high $4 \mathrm{e}^{-}$selectivity for Fe-N-C and high $2 \mathrm{e}^{-}$-selectivity for Co-N-C. The generally low selectivity of Co- $\mathrm{N}_{\mathrm{x}}$ sites for $4 \mathrm{e}^{-} \mathrm{ORR}$ is in line with the experience gained in ORR electrocatalysis by unpyrolysed $\mathrm{Co}_{-} \mathrm{N}_{4}$ macrocycles, where the adsorption of $\mathrm{OOH}$ on $\mathrm{Co}(\mathrm{II})$ is weak relative to that of $\mathrm{OOH}_{\text {ads }}$ intermediate on Fe(II). Beside the classical RRDE method, other methods have been developed to more locally probe tiny amounts of peroxide produced on surfaces or thin active films during ORR, such as the scanning electrochemical microscopy (SECM) $[133,134]$. In this approach, the position and electrochemical potential of a Pt tip (diameter of typically 20-30 $\mu \mathrm{m}$ ) situated directly above the ORR active surface is controlled (Figure $\mathbf{3 b}$ ). This SECM setup allows a shorter distance (typically 20-30 $\mu \mathrm{m}$ as well) between the ORR 
active surface and the Pt tip, where $\mathrm{H}_{2} \mathrm{O}_{2}$ is electro-oxidized to water, than is achievable with RRDE setups [135].

Another way to investigate the ability or not of Metal-N-C catalysts to indirectly electrocatalyse the ORR is to study their activity to electroreduce $\mathrm{H}_{2} \mathrm{O}_{2}$ to water, in the absence of ORR. This can be done in classical RDE setup, with a known concentration of $\mathrm{H}_{2} \mathrm{O}_{2}$ added into the electrolyte and bubbling with inert gas, in order to continuously remove $\mathrm{O}_{2}$ from the system. An early study showed the low activity of Fe-N-C and other Metal-N-C (with unknown metal speciation) for $\mathrm{H}_{2} \mathrm{O}_{2}$ electroreduction, when compared to Pt based electrocatalysts [136]. In particular, the polarisation curve for $\mathrm{H}_{2} \mathrm{O}_{2}$ electroreduction by all Metal-N-C in acid medium shows a linear shape, uncanny for electrocatalysis where exponential behaviours are expected, at least at low current density and in the absence of mass-transport limitation. Fe-N-C was found to be the most active for $\mathrm{H}_{2} \mathrm{O}_{2}$ electroreduction, followed by $\mathrm{Cu}-\mathrm{N}-\mathrm{C}$ [136]. A recent study investigated the $\mathrm{H}_{2} \mathrm{O}_{2}$ electroreduction in acid of three Fe-N-C catalysts with well identified Fe speciation, involving a Fe-N-C material free of any Fe clusters, a Fe-N-C material with a small amount of metallic Fe ( $c$ 8 rel. \%) along with Fe- $\mathrm{N}_{\mathrm{x}}$ sites, and a third Fe-N-C with a large fraction of Fe present as metallic iron and iron carbide (47 rel. \%) [130]. The results suggested that metallic Fe particles directly exposed to the electrolyte or metal-free N-C surface do not significantly catalyse $\mathrm{H}_{2} \mathrm{O}_{2}$ electroreduction in acidic medium. In contrast, both Fe- $\mathrm{N}_{\mathrm{x}}$ sites and metallic Fe particles wrapped in N-C protective shell seem to be able to catalyse both the direct $4 \mathrm{e}^{-} \mathrm{ORR}$ and the $\mathrm{H}_{2} \mathrm{O}_{2}$ electroreduction.

While $4 \mathrm{e}^{-}$ORR is desired in the context of fuel cell application, $\mathrm{H}_{2} \mathrm{O}_{2}$ is a useful chemical product and its selective synthesis via $2 \mathrm{e}^{-} \mathrm{ORR}$ is of potential interest to displace the current anthraquinone process used to produce it at scale. Until recently, platinum-mercury (Pt-Hg) and palladium-mercury (Pd-Hg) alloys or amalgams were the state-of-art electrocatalysts to that end, but suffer from high cost and environmental issues related to PGM and $\mathrm{Hg}$, respectively [137,138]. In a study on Metal-N-C materials with different 3d transition metals (Mn, Fe, Co, $\mathrm{Ni}$ and $\mathrm{Cu}$ ) but with all catalysts comprising only Metal- $\mathrm{N}_{\mathrm{x}}$ sites, Sun et al. found that Co-N-C results in the optimal balance between high selectivity for $2 \mathrm{e}^{-}$ORR and low overpotential [118]. While Fe-N-C has a slightly lower overpotential than Co-N-C and other Metal-N-C to form peroxide from $\mathrm{O}_{2}$, it also has a much higher activity to further electroreduce it to water. The 
latter aspect becomes increasingly a drawback, when the peroxide concentration increases over time, which is desired from an application viewpoint. The initial selectivity of Co-N-C catalysts towards $\mathrm{H}_{2} \mathrm{O}_{2}$ via $2 \mathrm{e}^{-} \mathrm{ORR}$ was further improved by intentionally introducing oxygen functional groups on the top surface [139]. In this approach, the negative effect of $\mathrm{H}_{2} \mathrm{O}_{2}$ in $\mathrm{PEM}$ fuel cells (namely, reducing the TOF and 4e- ORR selectivity of Fe-N-C and Co-N-C, see section 13.5) was brought to profit for modifying a Co-N-C catalyst aimed for $\mathrm{H}_{2} \mathrm{O}_{2}$ electrosynthesis. The high activity of Co-N-C for $2 \mathrm{e}^{-}$ORR combined with low activity for further electroreduction of $\mathrm{H}_{2} \mathrm{O}_{2}$ was explained at the atomic level by DFT, predicting an optimal $\mathrm{OH}$ binding energy on Co- $\mathrm{N}_{\mathrm{x}}$ sites, strong enough to bind $\mathrm{O}_{2}$ and $\mathrm{OOH}$ but not overly strong, allowing the desorption of $\mathrm{H}_{2} \mathrm{O}_{2}$ [118] instead of its further electroreduction. These features identify Co-N-C and other doped versions (doped with oxygen, etc.) as promising and affordable catalysts for the electrosynthesis of peroxide [140].

This section is concluded with a short discussion on the deconvolution of the overall ORR activity of a given Metal-N-C catalyst into its TOF and SD values, according to:

$$
\begin{gathered}
I_{m}=T O F \times S D_{m} \times F / N_{a} \\
I_{v}=T O F \times S D_{v} \times F / N_{a}
\end{gathered}
$$

Where $I$ is the ORR activity (mass- or volume-normalized, $\mathrm{m}$ or ${ }_{\mathrm{v}}$, respectively) at a given potential $E$, TOF the TOF at the same potential, SD the mass- or volume-normalized site density, $N_{\text {a }}$ Avogadro's number and $F$ Faraday's constant [141]. ORR volumetric activity targets were defined for the first time in 2005 for PGM-free catalysts, and deconvoluted into possible combinations of SD and TOF values in order to reach the same targeted overall ORR activity. Since then, methods have been developed that can quantify the SD, and hence (in combination with ORR activity measurement), the average TOF value of Fe-N-C catalysts $[126,128,129]$. While increasing the number of active sites seems a generally good idea to increase the total activity of an electrocatalyst, one would a priori assume the TOF to be relatively independent of the $\mathrm{SD}$ value. However, there is recent growing evidence that the TOF value of Metal- $\mathrm{N}_{\mathrm{x}}$ sites embedded in a nitrogen-doped carbon matrix is intimately related to the physico-chemical prop- 
erties of the latter. A recent benchmark study on four Fe-N-C catalysts revealed a negative correlation between TOF and SD values, i.e. decreasing TOF with increasing SD values of Fe- $\mathrm{N}_{\mathrm{x}}$ sites [59]. The doping of nitrogen-doped carbon with many Metal- $\mathrm{N}_{\mathrm{x}}$ sites may induce electronic and/or surface changes that reduce the TOF. As a second example of TOF changes, the introduction of oxygen surface groups on top of Fe-N-C after its synthesis has also been shown to decrease the TOF [112] (discussed in detail in section 13.5). The in-depth understanding of these inter-relations between TOF of Metal- $\mathrm{N}_{\mathrm{x}}$ sites and the physico-chemical properties of the nitrogen-doped carbon matrix (doped with other non-metal or metal elements) will be critical in order to combine high SD while retaining a high TOF in Metal-N-C materials in the future.

\section{2. $\mathrm{CO}_{2}$ reduction, $\mathrm{N}_{2}$ and $\mathrm{NO}_{3}^{-}$reduction}

The range of reactions that are catalysed by single-atoms Metal-N-C electrocatalysts is not limited to the four- or two-electron ORR, but also extends for example to the electrochemical reduction of $\mathrm{CO}_{2}\left(\mathrm{CO}_{2} \mathrm{RR}\right)$. $\mathrm{CO}_{2} \mathrm{RR}$ is of paramount importance for several reasons: (i) the industrial revolution and the resulting dramatic increase in $\mathrm{CO}_{2}$ in the atmosphere (from $250 \mathrm{ppm}$ in the early 1800 's maximum to 390 ppm nowadays [142]) are prime responsible of the greenhouse effect, thus making $\mathrm{CO}_{2}$ recapture essential to hinder global warming; (ii) $\mathrm{CO}_{2} \mathrm{RR}$ products are numerous, i.e. ranging from simple $\mathrm{CO}$ or formic acid $\mathrm{C}$ molecules to complex multicarbon molecules (here referred as $\mathrm{C}_{2+}$ ) obtained through direct electrochemical reduction or using cascade electrocatalysts (which consist in a combination of different catalysts each catalysing parts of the $\mathrm{CO}_{2} \mathrm{RR}$, that can be not only metallic or carbon-based, but also enzymatic, molecular, etc. [143]), each with their own added value. As such, the $\mathrm{CO}_{2} \mathrm{RR}$ has been under growing attention throughout the past decade, and the Metal-N-C electrocatalysts were investigated in details. The $\mathrm{CO}_{2} \mathrm{RR}$ is a complex reaction, which is in competition with the HER. Here arises the first advantage of the Metal-N-C electrocatalysts: As evidenced by Bagger et al., the Metal-N 4 sites are poorly active for the HER, as a result of (i) a different binding conformation (top $v s$. hollow) compared to extended metallic surfaces and (ii) a different, less favourable mechanistic pathway (Volmer-Heyrovsky vs. Volmer-Tafel) which overall leads to a $0.3 \mathrm{eV}$ energy penalty for the HER for single-atom sites vs. metallic surfaces [144]. Owing to this depreciated activity for the HER, the Metal-N-C electrocatalysts are highly selective for the $\mathrm{CO}_{2} \mathrm{RR}$, but the nature of its products on said electrocatalysts needs to be discussed. 

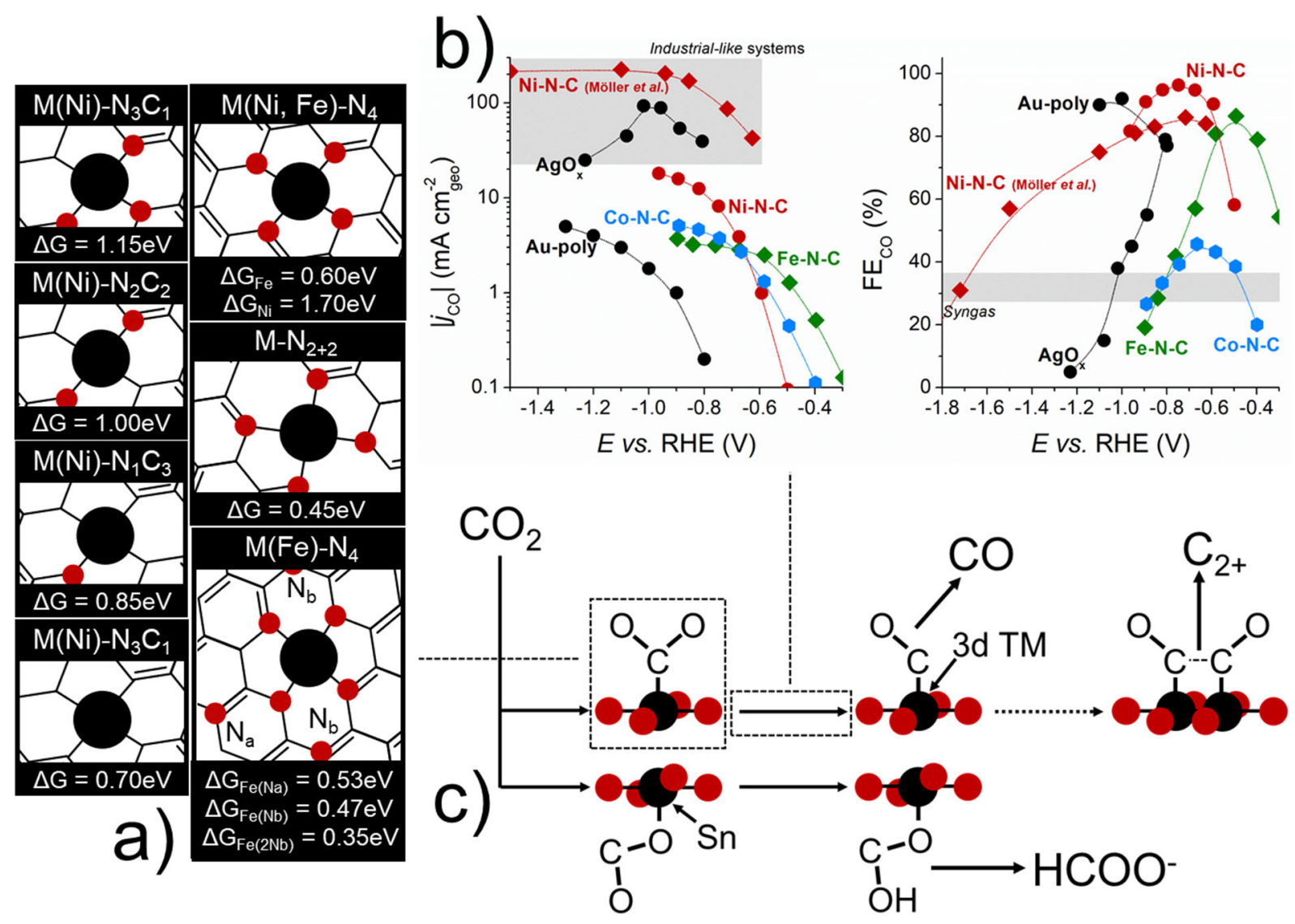

Figure 4. Nature of the active sites, activity and reaction paths for the electrochemical reduction of $\mathrm{CO}_{2}$ by metal-nitrogen-carbon catalysts. (a) illustration of various Metal- $\mathrm{N}_{\mathrm{x}}$ moieties investigated by DFT and the free energy transition for the first proton + electron transfer $\left(\mathrm{CO}_{2} \rightarrow \mathrm{COOH}_{\mathrm{ads}}\right)$; (b) partial current density for $\mathrm{CO}$ generation and faradaic efficiency for $\mathrm{CO}$ generation for various MetalN-C electrocatalysts originating from the work of Pan et al. [145] and Möller et al. [146], along with polycrystalline $\mathrm{Au}$ (Hori et al. [147]) and $\mathrm{AgO}_{\mathrm{x}}$ (Möller et al. [146]) and (c) illustration of the mechanistic pathway on early transition $3 \mathrm{~d}$ Metal-N-C and $\mathrm{Sn}-\mathrm{N}-\mathrm{C}$ for the electrochemical reduction of $\mathrm{CO}_{2}$, along with an hypothetical pathway in presence of dual-atoms moieties. Source: Tristan Asset, Frédéric Maillard, Frédéric Jaouen.

When addressing the $\mathrm{CO}_{2} \mathrm{RR}$ on metallic extended surfaces, the $\mathrm{CO}_{2} \mathrm{RR}$ products can be categorized in three groups, excluding the elements that are more selective for $\mathrm{H}_{2}$ generation [148]: (i) $\mathrm{CO}$; (ii) formic acid (if the $\mathrm{CO}_{2}$ adsorb through one of its oxygen atoms instead of the carbon atoms [149]) and, on Cu-based electrocatalysts, (iii) $\mathrm{C}_{2+}$ (including $\mathrm{C}_{2} \mathrm{H}_{4}$, ethanol, etc.), although with a low selectivity for a given product. Metal-N-Cs mainly belong to the first category, 
due to (i) the initial adsorption of $\mathrm{CO}_{2}$ through its carbon atom and (ii) the fact that the adsorption of two adsorbed $\mathrm{CO}\left(\mathrm{CO}_{\mathrm{ads}}-\mathrm{CO}_{\mathrm{ads}}\right)$ on a single Metal- $\mathrm{N}_{4}$ moiety is highly unfavourable $\left(\Delta \mathrm{G}_{\text {COads }} \rightarrow\right.$ COads-COads $\left.=c a 0.7 \mathrm{eV}[150]\right)$, thus greatly limiting the Metal-N-Cs capability to form a $\mathrm{C}-\mathrm{C}$ bond and thus generate $\mathrm{C}_{2}$ species. Despite the simple nature of its main product, i.e. $\mathrm{CO}$, performing the $\mathrm{CO}_{2} \mathrm{RR}$ on Metal-N-C SMAC remains of interest: the $\mathrm{CO}$ can be combined with $\mathrm{H}_{2}$ to generate syngas. The latter is a $c a 1: 2$ combination of $\mathrm{CO}$ and $\mathrm{H}_{2}$ and an intermediate in the synthesis of complex carbon-based molecules through the Fischer-Tropsch process [151]. It is currently mainly produced from fossil fuels, thus making the $\mathrm{CO}_{2} \mathrm{RR}$ on Metal-N-Cs electrocatalysts a viable alternative for syngas generation.

First, it is important to limit the scope of this discussion on Metal-N-C electrocatalysts, i.e. pyrolysed materials presenting atomically dispersed metallic atoms surrounded by nitrogen, embedded in the carbon structure, therefore excluding carbon-supported molecular catalysts, chalcogenides and other non-pyrolysed materials [152], although the latter also presented relevant performances for the $\mathrm{CO}_{2} \mathrm{RR}$ (e.g. a faradaic efficiency $>80 \%$ toward $\mathrm{CO}$ was recently reported by Pan et al. on Co phthalocyanine on nitrogen-doped porous carbon nanospheres [153]). However, they remain limited in terms of catalytic activity vs. the Metal-N-Cs, and exhibit a lower stability due to the non-embedded nature of the active sites. It is also important to note that as a result of their synthesis process, the Metal-N-Cs do not solely exhibit Metal$\mathrm{N}_{4}$ sites as their catalytic sites. As discussed in Section 13.3, three potential types of actives sites can coexist in such materials, namely: (i) the metal-free, nitrogen-containing sites (e.g. Npyridinic, N-pyrrolic and N-graphitic), (ii) the metallic NP and clusters and (iii) the Metal- $\mathrm{N}_{\mathrm{x}}$ sites. Owing to the focus of this chapter, (i) and (ii) will only be superficially discussed, to mainly focus on SMA sites. First, the role of Metal-free N-moieties to the electrocatalytic activity of Metal-N-C electrocatalysts is non-negligible: (i) an entire sub-field of the $\mathrm{CO}_{2} \mathrm{RR}$ is exclusively dedicated to the study of nitrogen-doped carbon [154-156], which often exhibit great faradaic efficiency at low overpotentials (e.g. $\mathrm{FE}_{\mathrm{CO}}=80 \%$ on nitrogen-doped carbon nanotubes or graphene); (ii) it was also observed that, on Fe-N-Cs, the activity was increasing with the N-pyridinic abundancy and that those moieties acted as preferential $\mathrm{CO}_{2}$ chemisorption sites [150]. On the other hand, the presence of metallic NP/nanocluster leads to a decrease of the faradaic efficiency toward CO, and catalyse the HER, as recently shown by Huan et al. [100]. 
The reactivity of the Metal- $\mathrm{N}_{\mathrm{x}}$ moieties in Metal-N-C for the $\mathrm{CO}_{2} \mathrm{RR}$ was explored through two main axes: (i) achieving an understanding of the reactivity of metal-containing moieties (Metal$\mathrm{N}_{4}$, Metal- $\mathrm{N}_{2+2}$, etc. some of which are illustrated in Figure 4a) towards the $\mathrm{CO}_{2} \mathrm{RR}$ through DFT and (ii) experimental assessment of various Metal-N-C electrocatalysts. For Metal-N-Cs, the rate determining step of the $\mathrm{CO}_{2} \mathrm{RR}$ is the first electron transfer of the $\mathrm{CO}_{2}$ reduction in $\mathrm{COOH}_{\text {ads }}\left(\mathrm{CO}_{2} \rightarrow \mathrm{CO}_{2}{ }^{-}\right)[157,158]$, and the role played by the nature of the metal element of an Metal-N-C electrocatalyst, under its Metal-N 4 form, on the free energy transition $(\Delta G)$ has been widely studied. Early $3 \mathrm{~d}$ transition metals $\left(\mathrm{Cr}-\mathrm{N}_{4}, \mathrm{Mn}-\mathrm{N}_{4}, \mathrm{Fe}-\mathrm{N}_{4}, \mathrm{Co}-\mathrm{N}_{4}\right)$ only require energies ranging from 0.25 to $0.60 \mathrm{eV}$ to achieve the complete step $\left(\mathrm{CO}_{2} \rightarrow \mathrm{COOH}\right)$, whereas for $\mathrm{Ni}-\mathrm{N}_{4}$ and $\mathrm{Cu}-\mathrm{N}_{4} \Delta \mathrm{G}$ range up to $1.60 \mathrm{eV}$. The metal element nature also impacts the $\mathrm{CO}_{\text {ads }}$ binding strength. The $3 \mathrm{~d}$ early transition metals Metal- $\mathrm{N}_{4}$ moieties act as strong $\mathrm{CO}_{\text {ads }}$ binders, whereas Ni-N $4, \mathrm{Cu}-\mathrm{N}_{4}$ and $\mathrm{Zn}-\mathrm{N}_{4}$ spontaneously desorb CO below $0 \mathrm{~V}$ vs. RHE [150]. These theoretical considerations predict fairly well the trends observed for the $\mathrm{CO}_{2} \mathrm{RR}$ activity and selectivity of various Metal-N-Cs electrocatalysts, as shown in Figure 4b. For example, Ni-N$\mathrm{C}$ electrocatalysts present a high $\mathrm{CO}_{2} \mathrm{RR}$ onset potential, along with up to $90-100 \%$ faradaic efficiency for $\mathrm{CO}$ generation, which arise from (i) the high free energy for hydrogen adsorption on Ni-N 4 , (ii) the high $\Delta \mathrm{G}$ for the first electron transfer in the $\mathrm{CO}_{2} \mathrm{RR}$ and (iii) the weak binding of the $\mathrm{CO}_{\mathrm{ads}}[88,145,159]$. On the other hand, Co-N-C shows lower selectivity $(20-40 \%)$ for $\mathrm{CO}$ generation, as a result of its far lower $\Delta \mathrm{G}$ for the $\mathrm{H}^{+} \rightarrow \mathrm{H}_{\text {ads }}$ HER step [88]. The reactivity and selectivity of the metal-containing moieties also depends of their environment, coordination and the oxidation degree of the metal. Removing nitrogen atoms from the Metal- $\mathrm{N}_{4}$ structure, or replacing carbon by nitrogen in the neighbouring benzene cycle was investigated by DFT and was shown to greatly impact the $\Delta \mathrm{G}$ of the $\mathrm{CO}_{2} \rightarrow \mathrm{COOH}_{\text {ads }}$ step [160]. For example, whereas a Ni-N 4 site exhibit a $\Delta \mathrm{G}=1.7 \mathrm{eV}$ for the first electrochemical step, a Ni-C4 site should only exhibit a $\Delta \mathrm{G}=0.7 \mathrm{eV}$ [146]. The free energy variations were also impacted by other changes in the moiety' conformation, e.g. switching toward Metal-N 3 sites or transitioning toward bridges sites (Metal- $\mathrm{N}_{2+2}$ ) [161]. Those different structures are summarized in Figure 4a. Notably, these structures were studied through DFT. An experimental catalyst designed through the approaches discussed in Section 13.2 is unlikely to exhibit a single, optimal, type of Metalcontaining moieties, i.e. usually several moieties are simultaneously present in a Metal-N-C catalyst, such as the Metal- $\mathrm{N}_{4}$ and the Metal- $\mathrm{N}_{3}$ in the basal plane, the Metal- $\mathrm{N}_{2+2}$ as 'bridge' 
over small pores (see Section 13.3.1.1), etc. Beyond its impact on the $\Delta \mathrm{G}$, the conformation of these moieties modifies the binding strength of given intermediates e.g. $\mathrm{CO}_{\text {ads. }}$ The relative abundancy of the metal-containing moieties therefore impacts the $\Delta \mathrm{G}$ for the first electrochemical step, along with the binding energy of the intermediates species and can be tuned by modifying the Metal-N-C morphology, e.g. a more microporous electrocatalyst leads to a higher proportion of Metal- $\mathrm{N}_{2}+2$, whereas a mesoporous electrocatalyst exhibit a higher proportion of basal planes in the mesopores, and, thus, of Metal-N4. A practical example of the impact of the morphology onto the electrocatalyst selectivity is found in the recent work of Hursàn et al., which evidenced that the faradaic efficiency toward $\mathrm{CO}_{2} \mathrm{RR}$ (vs. HER) on nitrogen-doped porous carbon was directly correlated to the pore size [162].

Both from a theoretical and experimental standpoint, Metal-N-C electrocatalysts are extremely promising for syngas generation due to the limited number of products, i.e. almost exclusively $\mathrm{H}_{2}$ and $\mathrm{CO}$, and their high selectivity toward the latter, especially for Ni-N-C. This selectivity can be finely tuned by modifying the nature and chemistry of the main active sites, e.g. the Metal- $\mathrm{N}_{\mathrm{x}}$ sites, or by adding metallic NP, along with the porous network of the electrocatalyst, hence allowing the production of syngas with various $\mathrm{CO}: \mathrm{H}_{2}$ ratios. However, such electrocatalysts should not be considered on the sole basis of their expected performances, or the $\mathrm{CO}_{2} \mathrm{RR}$ performances obtained in liquid, static, electrolyte, but already considered as to-be commercial electrocatalysts and designed in that frame. Recently, notable efforts have been carried to transfer this technology toward zero-gap electrolyzer [163] (i.e. systems where the electrocatalysts are in direct contact with the membrane, not separated by an electrolyte layer, static or circulating) or flow cells, leading to performances up to $200 \mathrm{~mA} \mathrm{~cm}^{-2}$ geo on Ni-N-C reported by Möller et al. [146]. Transferring from a liquid to a solid electrolyte induces new design-related challenges, and further optimization of the electrocatalyst pores distribution, hydrophobicity, etc. Thankfully, the wide range of synthesis process available to achieve Metal-N-C electrocatalysts greatly facilitate the tuning of such parameters (see Section 13.2), e.g. use of different metalorganic-frameworks or silica spheres in the soft and hard templating methods, respectively; use of different carbon precursors, etc. An important question is now whether the selectivity of

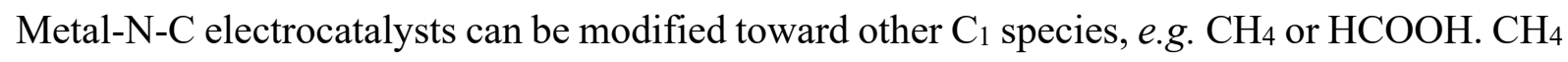
generation is sometimes observed on Metal-N-C, but remains a minor product $(<1 \%)$, with an enhanced selectivity at $E<-1.1 \mathrm{~V} v s$. RHE on Fe-N-C, coincidental with a modification of the 
Fe-N 4 oxidation degree, from $\mathrm{Fe}(\mathrm{II})-\mathrm{N}_{4}$ to an hypothesized $\mathrm{Fe}(\mathrm{I})-\mathrm{N}_{\mathrm{x}}[164]$. The formation of $\mathrm{C}_{2}$ species, such as ethanol (up to $40 \%$ at $-1.2 \mathrm{~V} v s$. RHE), was reported on a Cu-N-C SMAC [165]. However, this unexpected $\mathrm{CO}_{2} \mathrm{RR}$ product for SMA sites was shown to be related to the rapid operando formation of metallic $\mathrm{Cu}$ clusters from $\mathrm{Cu}-\mathrm{N}_{\mathrm{x}}$ sites during $\mathrm{CO}_{2} \mathrm{RR}$. Interestingly, more opportunities exist for $\mathrm{HCOOH}$ production with Metal-N-C. On extended surfaces or NP, Sn-based electrocatalysts reach $80 \%$ faradaic efficiency for formate generation [166,167], making them natural candidates for investigating the $\mathrm{CO}_{2} \mathrm{RR}$ reactivity of tin in Sn-N-C. Recent work from $\mathrm{Zu}$ et al. [168] and Paul et al. [169] focused on the design and utilization of Sn-N$\mathrm{C}$ and reported faradaic efficiency toward formate generation up to $75 \%$, owing to the stabilization of the $\mathrm{CO}_{2}{ }^{-}$intermediates on the $\mathrm{Sn}-\mathrm{N}_{\mathrm{x}}$ moieties. Finally, achieving $\mathrm{C}_{2}$ products on non$\mathrm{NP}$ based electrocatalysts requires the adsorption of $\mathrm{CO}_{\text {ads }}$ onto neighbouring sites to alloy the formation of the $\mathrm{C}-\mathrm{C}$ bond, and, therefore, the design of sites alloying such conformation, e.g. dual-atoms sites $\left(\mathrm{Fe}_{2}-\mathrm{N}_{\mathrm{x}}, \mathrm{Fe}-\mathrm{Co}-\mathrm{N}_{\mathrm{x}}\right.$, etc.). They possibly already exist in minor proportions in Metal-N-C but are extremely challenging to characterize, along to synthesize as the sole type of sites of a Metal-N-C electrocatalyst. Those different points are illustrated in Figure 4c.

The attractiveness of Metal-N-C electrocatalysts for electroreduction extends toward other complex reactions, e.g. the nitrogen reduction reaction $\left(\mathrm{N}_{2} \mathrm{RR}\right)$. The $\mathrm{N}_{2} \mathrm{RR}$ aims at the electrochemical production of ammonia $\left(\mathrm{NH}_{3}\right)$, at near-room temperature and near-ambient pressure. The latter challenges arise from (i) the energy stored in the $\mathrm{N} \equiv \mathrm{N}$ bond $\left(941 \mathrm{~kJ} \mathrm{~mol}^{-1}\right)$ and (ii) the large band gap $(10.82 \mathrm{eV})$ between the highest unoccupied molecular orbital (HUMO) and the lower occupied molecular orbital (LOMO) [170]. This explains the extreme conditions under which it is usually performed, i.e. through the Haber-Bosch process at $300-500{ }^{\circ} \mathrm{C}$ and $200-300$ bars on Fe-based catalysts. The Metal-N-C (M = Fe, Mo, etc.) were investigated for the $\mathrm{N}_{2} \mathrm{RR}$, taking inspiration from both the Haber-Bosch electrocatalysts and the nitrogenase (an enzymatic complex existing in the nature, able to perform the $\mathrm{N}_{2} \rightarrow \mathrm{NH}_{3}$ through the use of a $\mathrm{Fe}_{4} \mathrm{~S}_{4}$-based complex and a molybdenum-iron protein [171]) and advantage of the low HER activity of Metal-N-C electrocatalysts. The choice of those specific elements also arises from the work from Skúlason et al., which identified those elements, along with ruthenium and rhodium as the thermodynamically most promising for $\mathrm{N}_{2} \mathrm{RR}$ [172]. This results from their nearoptimal $\mathrm{N}_{\text {ads }}$ binding (leading to an $\mathrm{N}_{2} \mathrm{RR}$ thermodynamically favourable at $E v s$. RHE $<-0.5$ V). However, the same work underlined the far more favourable $\mathrm{H}_{\text {ads }}$ adsorption $v s . \mathrm{N}_{\text {ads }}$ and 
the subsequent easier nature of the HER $v s . \mathrm{N}_{2} \mathrm{RR}$ on a wide variety of extended surfaces. This gap cannot be closed by the $0.3 \mathrm{eV}$ penalty brought by the use of SMAC. Hence, despite promising faradaic efficiency for $\mathrm{NH}_{3}$ generation reported in the literature on Metal-N-C electrocatalysts (with a maximum of $55 \%$ on Fe-N-C) [173-175], it is inferred that the $\mathrm{NH}_{3}$ produced by those materials does not originate from the $\mathrm{N}_{2} \mathrm{RR}$, but from other sources. Throughout the past years, a tremendous effort has been carried out to investigate the alternative sources of $\mathrm{NH}_{3}$ when investigating the electrochemical $\mathrm{NH}_{3}$ synthesis, not only for Metal-N-C, but for all electrocatalysts families', in order to avoid false positives. In that frame, two principal sources were identified (i) $\mathrm{NH}_{3}$ contamination from neighbouring contaminations, e.g. electrolyte, gas, air, which is usually addressed by using ${ }^{15} \mathrm{~N}_{2}$ gas as a reactant (the $\mathrm{NH}_{3}$ produced by the $\mathrm{N}_{2} \mathrm{RR}$ being then ${ }^{15} \mathrm{NH}_{3}$ ) and (ii) the presence of $\mathrm{NO}_{3}$ impurity as a minor species in ${ }^{14} \mathrm{~N}_{2}$ and ${ }^{15} \mathrm{~N}_{2}$ gas sources [176-178]. If the $\mathrm{NH}_{3}$ generation observed with Metal-N-C arises from the $\mathrm{NO}_{3}$ impurities reduction and not from $\mathrm{N}_{2}$ this implies that they may exhibit a low activity for the $\mathrm{N}_{2} \mathrm{RR}$, but might be promising for the $\mathrm{NO}_{3} \mathrm{RR}$. This reaction recently encountered a renewed interest [179], as an alternative to the Haber-Bosch process as an $\mathrm{NH}_{3}$ source and thus act as a natural direction for future investigation of the electrocatalytic properties of Metal-N-C electrocatalysts.

\section{Stability of Metal-N-C electrocatalysts}

As three chemical elements, metal, nitrogen and carbon, constitute the active sites in Metal-NC electrocatalysts, it can thus be intuitively perceived that any change in the electronic/physical structure of one of these elements can modify the TOF of the active sites or lead to their complete destruction. At least five degradation mechanisms of Metal-N-C catalysts have been identified: (i) demetallation, (ii) changes in the chemical and physical nature of the central metal ion, (iii) protonation of the $\mathrm{N}$-groups, (iv) reversible or irreversible corrosion of the carbon matrix (carbon oxidation reaction - COR) and associated phenomena such as (v) migration and aggregation of the SMAs (Figure 5). The predominant degradation mechanism of SMAC strongly depends on the nature of the potential range, the temperature and the nature of the atmosphere, and these experimental conditions will be detailed in what follows. 


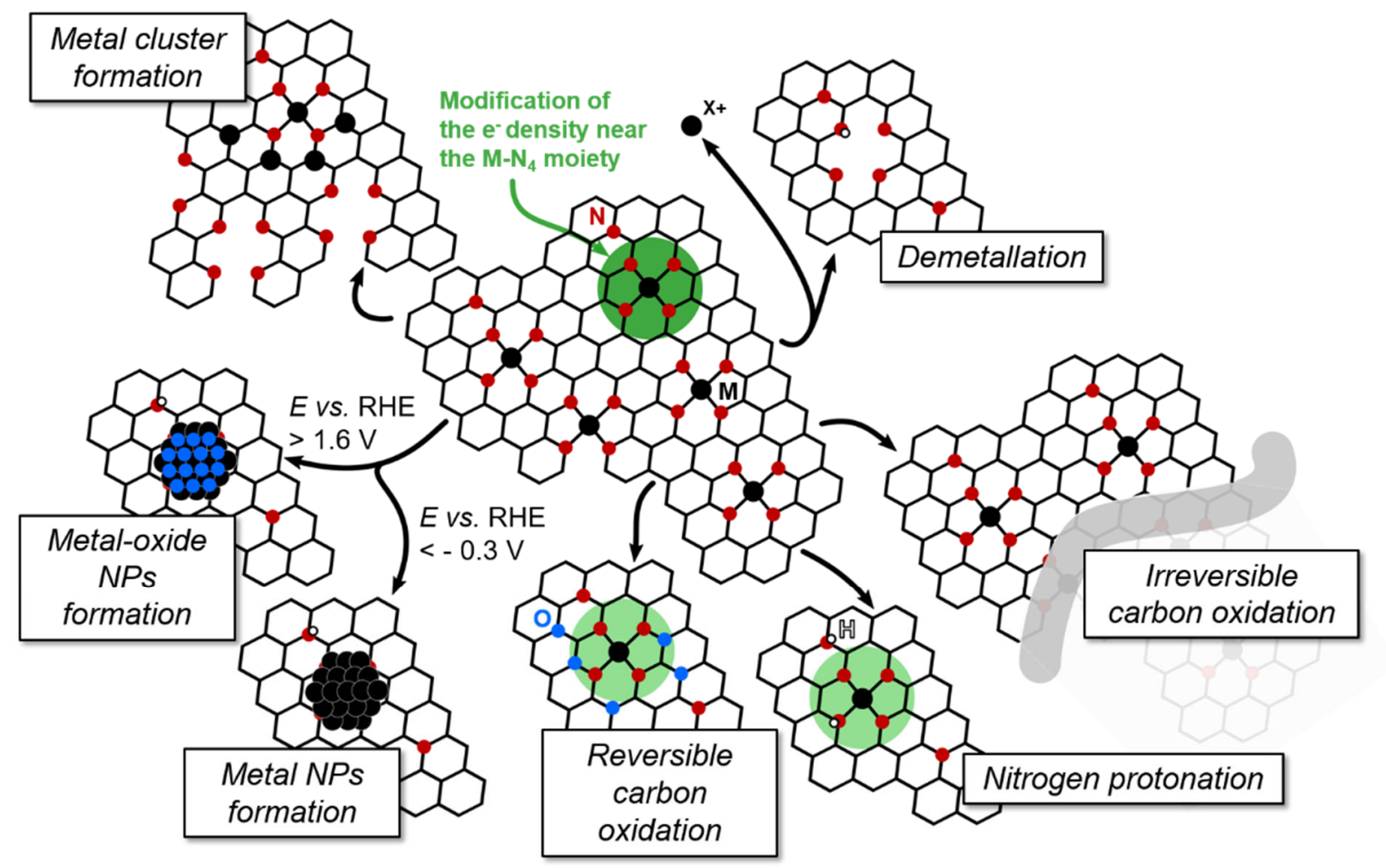

Figure 5. Illustration of the main degradation processes occurring during a Metal-N-C electrocatalyst operation, i.e. demetallation, reversible or irreversible carbon oxidation reaction, nitrogen protonation and metal clusters formation. The intensity of the colour of the green circles is indicative of the electron density near the M-N 4 moiety. Source: Tristan Asset, Frédéric Maillard, Frédéric Jaouen.

\subsection{Demetallation in the absence of carbon oxidation}

Demetallation of SMAC easily occurs upon corrosion of the carbon matrix [98,115]. This degradation mechanism will be covered in the following sub-sections, and we focus here on the demetallation of SMAC in the absence of COR. Pourbaix diagrams, which represent the domain of stability of different phases of an element as a function of $\mathrm{pH}$, potential range (in Pourbaix diagrams, the potential is given with respect to the standard hydrogen electrode, SHE) are powerful tools to estimate the resistance to demetallation of Metal-N-C SMAC. In particular, Pourbaix diagrams reveal that acidic $\mathrm{pH}$, high potential and $\mathrm{O}_{2}$ are very aggressive stressors for SMAC. These conditions are typical of the operating conditions of a PEMFC cathode, in which metal cations ( $\mathrm{Fe}(\mathrm{II}) / \mathrm{Fe}(\mathrm{III}), \mathrm{Co}(\mathrm{II}), \mathrm{Mn}(\mathrm{II})$ and $\mathrm{Cr}$ (III) cations) are the stable phases of $\mathrm{Fe}, \mathrm{Co}$, $\mathrm{Mn}$ and $\mathrm{Cr}$. There are two driving forces for demetallation: (i) competition between the metallic ion in the Metal-N-C catalyst and protons for the $\square-\mathrm{N}_{\mathrm{x}}$ (i.e. nitrogen structures where the metal 
cation is removed), (ii) change in the oxidation state of the SMA. In the frame of the first scenario, there is a risk of demetallation when the SMAC are exposed to cations with higher ionic potential (the ratio of the charge density to the radius of the atom over which this charge is distributed) than the central metal cation. As the ionic radius of $\mathrm{H}^{+}$is $134 \mathrm{pm}$ and that of $\mathrm{Fe}(\mathrm{II}) / \mathrm{Fe}(\mathrm{III})$ cations is $77 / 63 \mathrm{pm},[180]$ the higher ionic potential of Fe cations provides thermodynamic stability to Fe-N-C SMAC in acidic electrolyte. The same reasoning applies to $\mathrm{Co}$ (III) (68.5 pm), $\mathrm{Mn}$ (II) (80 pm) and $\mathrm{Cr}$ (III) (75.5 pm) cations. Hence, the demetallation observed at the beginning of life of some Metal-N-C catalysts may simply be perceived as improper stabilization of SMAC (incomplete coordination of metal cations to $\mathrm{N}_{\mathrm{x}}$ atoms) or dissolution of some NP (carbides, nitrides, sulphides, oxides depending on the synthesis conditions) present in the catalyst, and which are more prone to dissolution than SMA [98,115]. Demetallation is also prone to occur because the oxidation state of the central metal ion changes during electrocatalysis. In this frame, metal porphyrins and related derivatives such as metal-phthalocyanines (Metal-Pc) have often been studied to gain insights into the electrocatalytic activity and the stability of Metal-N-C catalysts. Baranton et al. reported drop in ORR activity on FePc after holding the potential at $0.5 \mathrm{~V} v s$. RHE or upon cycling between 0.05 and $0.90 \mathrm{~V} v s$. RHE in $\mathrm{O}_{2}$-saturated $0.5 \mathrm{M} \mathrm{H}_{2} \mathrm{SO}_{4}$ [181]. The authors suggested that the electron transfer from the out-of-plane $\mathrm{Fe}(\mathrm{II})$ to the adsorbed $\mathrm{O}_{2}$ molecule results in a transient $\mathrm{Fe}(\mathrm{III})$ state, destabilized in acid medium due to its lower ionic radius than Fe(II), and they backed up their findings with infrared (IR) spectroscopy measurements showing formation of the protonated, metal-free macrocycle $\left(\mathrm{H}_{2}-\mathrm{Pc}\right)$. Similar observation and explanation have been earlier proposed by Meier et al. for polymeric Fe(II)-Pc [182]. However, no change of IR spectra was observed in Arsaturated $0.5 \mathrm{M} \mathrm{H}_{2} \mathrm{SO}_{4}$, thus indicating that the lack of stability of Fe-Pc is not related to oxidation of $\mathrm{Fe}(\mathrm{II})$ in $\mathrm{Fe}(\mathrm{III})$ but to ORR electrocatalysis. A change from $\mathrm{Fe}-\mathrm{Pc}$ to $\mathrm{H}_{2}-\mathrm{Pc}$ was also reported by Chen et al. upon stepping to $0.4 \mathrm{~V} v s$. RHE in aerated $0.1 \mathrm{M} \mathrm{HClO}_{4}$, not in $\mathrm{N}_{2}-$ saturated electrolyte, using operando tip-enhanced Raman spectroscopy (EC-TERS) [183]. Similarly, Kumar et al. showed that the active sites of Fe-N-C and Co-N-C SMAC remained unchanged after 10,000 potential steps between $0.6-1.0 \mathrm{~V}$ vs. RHE in Ar-saturated $0.1 \mathrm{M}$ $\mathrm{H}_{2} \mathrm{SO}_{4}$ thermostated at $80{ }^{\circ} \mathrm{C}[115,127]$. In contrast, the same AST performed in $\mathrm{O}_{2}$-saturated $0.1 \mathrm{M} \mathrm{H}_{2} \mathrm{SO}_{4}$ leads to formation of $\mathrm{Fe}$ nano-oxides. The pivotal role of $\mathrm{O}_{2}$ adsorption and dissociation on demetallation will be discussed in details in Section 5.6. 


\subsection{Changes in the chemical and physical nature of the metal ion: Metal-N-C as a pre- catalyst}

Although Metal-N-C SMAC are stable over a wide range of potential in acidic and alkaline electrolytes, their atomic-scale structure and chemistry change significantly in highly reductive $(E<-0.3 \mathrm{~V} v s$. SHE) and highly oxidative $(E>1.5 \mathrm{~V} v \mathrm{~s}$. SHE) conditions such as those encountered during the HER/CO $\mathrm{C}_{2} \mathrm{RR} / \mathrm{N}_{2} \mathrm{RR}$ and the OER, respectively. Consequently, the question arises whether the active site identified ex situ remains identical under reaction conditions. Based on Pourbaix diagrams, the stable phase of many transitions metals is higher oxidation state or metal oxide in acidic or alkaline OER conditions, respectively. Recently, Bai et al. reported that a Co-N-C SMAC can be transformed into a Co-Fe double atom catalyst that is highly active towards the OER in alkaline electrolyte [184]. High OER activity nevertheless involved multiple preliminary steps: a Co-N-C catalyst was first synthesized, and then pretreated ('activated') in Fe-containing $0.1 \mathrm{M} \mathrm{KOH}$, leading to formation of Co-Fe bonds as revealed from EXAFS. The OER activity rapidly declined over time but could be restored upon reactivation in Fe-containing $0.1 \mathrm{M} \mathrm{KOH}$, thus providing evidence for the key role of $\mathrm{Fe}$ in alkaline OER catalysis. Upon long-term electrolysis, the oxidation state of Co atoms changed from $\mathrm{Co}(\mathrm{II})$ to $\mathrm{Co}(\mathrm{IV})$ as revealed from EXAFS, which the authors ascribed to formation of $\mathrm{Co}(\mathrm{IV})=\mathrm{O}$ bonds. No TEM image of the aged material was provided, thus raising doubts about the nature of the active sites. Indeed, by combining EXAFS and TEM, Hocking et al. reported that tetranuclear-Mn cluster $\left[\mathrm{Mn}_{4} \mathrm{O}_{4} \mathrm{~L} 6\right]^{+}(\mathrm{L}=$ diarylphosphinate) transform in 5-10 nm Mnoxide particles after polarization at $1.0 \mathrm{~V} v s . \mathrm{Ag} / \mathrm{AgCl} / 3 \mathrm{M} \mathrm{NaCl}$ (potential of $0.2 \mathrm{~V} v s$. the SHE at $25^{\circ} \mathrm{C}$ ) in $0.1 \mathrm{M} \mathrm{Na}_{2} \mathrm{SO}_{4}$ electrolyte [185]. These results must be also compared with those of Kanan and Kocera [186], who reported that extremely active OER catalysts of micrometresize dimension form upon polarization of tin oxide electrode in phosphate-buffered water containing $\mathrm{Co}^{2+}$ ions. It may thus be proposed that Metal-N-C do not electrocatalyse the OER but rather act as a 'pre-catalyst' in OER conditions, providing metal ions for the formation of the catalytically active metal oxides. Pourbaix diagrams also indicate that $3 \mathrm{~d}$ transition metals are prone to reduce to their metallic form at a modest cathodic potential, such as that employed to electrocatalyse the HER and the $\mathrm{CO}_{2} \mathrm{RR}$. Anxolabéhère-Mallart et al.[187] showed that metallic Co NP form from Co bis glyoximato complexes during HER in aprotic solvents (acetonitrile + $0.1 \mathrm{M} \mathrm{HClO}_{4}$ in this study), independently on the initial ligands [188,189]. Similarly, Karapinar 
et al. evidenced that $\mathrm{Cu}(\mathrm{II})$ species are reduced into $\mathrm{Cu}(\mathrm{O})$ (metallic $\mathrm{Cu}$ ) in the reductive conditions of the $\mathrm{CO}_{2} \mathrm{RR}$ in close to neutral $\mathrm{pH}$, as mentioned in Section 13.4.2 [165]. Using EXAFS, the authors showed that the change in oxidation state was reversible, however $\mathrm{Cu}$ NP not $\mathrm{Cu}-\mathrm{N}-\mathrm{C}$ were considered to be the active sites for the $\mathrm{CO}_{2} \mathrm{RR}$, in line with former conclusions of Weng et al. [190]. These findings underline the importance of operando measurements, and nicely illustrate that Metal-N-C shape the formation of metallic or oxidized NP in highly reductive or oxidative conditions.

\subsection{Protonation of nitrogen atoms}

Protonation of $\mathrm{N}$-groups in acidic media is another well-established deactivation mechanism of Metal-N-C catalysts, especially for catalysts pyrolysed under $\mathrm{NH}_{3}\left(\mathrm{NH}_{3}\right.$ increases the basicity of the N-C matrix $[73,191])$. The Popov's group $[192,193]$ argued that N-pyridinic species are more prone to protonate in acidic media due to their lone pair of electrons. In contrast, catalysts featuring mainly N-graphitic perform initially worse towards the ORR but are more stable [192]. According to Herranz et al., these are the nitrogen groups located close to the Metal- $\mathrm{N}_{\mathrm{x}}$ sites (hence not directly participating to the ORR), which are protonated thus leading to indirect deactivation of the active sites [194]. The authors operated a PEMFC containing an acidleached Fe-N-C catalyst at the cathode, and reported a drop in performance that they related to protonation of the nitrogen groups quickly followed by anion adsorption. Importantly, such protonation proved to be partially or totally reversible, i.e. part of the catalyst's activity could be restored after an ex situ thermal or chemical treatment. Now, besides the deactivation of the catalytic sites, it should be mentioned that protonated nitrogen groups poorly transport $\mathrm{H}^{+}$, due to weakened ionomer-catalyst interactions [195]. In conclusion, the protonation of the nitrogen groups partially accounts for the ORR activity decay of some Metal-N-C catalysts but is not a real degradation mechanism per se.

\subsection{Carbon oxidation reaction}

The carbon matrix ensures electron conduction and transport of reactants to and products from the electrode, and is compatible with most of the ionomers required for efficient ion transport in electrochemical devices. Thermodynamically speaking, the carbon matrix can be oxidized in highly acidic conditions ( $\mathrm{pH} 0-1$ ) to $\mathrm{CO}_{2}$ or $\mathrm{CO}$ at $E \geq 0.21 \mathrm{~V} v s$. SHE and $E \geq 0.52 \mathrm{~V} v s$. SHE, 
respectively. Kinetically speaking, the COR rate is negligible below 1.0 V vs. SHE [196,197] but it increases with temperature (especially above $50^{\circ} \mathrm{C}$ ) [197-199], and at high relative humidity [200-202]. Mild COR leads first to the formation of oxygen-containing groups on the surface of the carbon matrix [203]. As the TOF of Metal- $\mathrm{N}_{\mathrm{x}}$ moieties strongly depends on their local environment (coordination number and electronegativity of the neighboring atoms $[112,204,205]$, these oxygen-containing surface groups lead to the depletion of the delocalized $\pi$ electrons and result in a drop of the TOF of Metal-N-C catalysts. For example, recent combined DFT calculations and experimental work by Jung et al. have shown that hydrogen or oxygen-containing carbon surface groups change the binding strength of transition metal to $\mathrm{OOH}_{\text {ads }}$ species, and control the ORR selectivity [139]. Using these findings, the authors selectively synthesized $\mathrm{H}_{2} \mathrm{O}_{2}$ on Co atoms dispersed on oxidized graphene catalysts. A consequence of the formation of oxygen-containing surface groups is that the carbon surface turns from hydrophobic to hydrophilic [206]. According to Zhang et al. [203] and Wang et al. [207], hydrophilic carbon surfaces are more prone to micropores' flooding, and beside demetallation and/or transformation of $\mathrm{Fe}-\mathrm{N}_{\mathrm{x}}$ sites into Fe-oxides, mild COR might also partially account for the rapid decay in performance observed on Fe-N-C catalysts, for example after a cell voltage hold at $U=0.5 \mathrm{~V}$ for 100 hours in a $\mathrm{H}_{2} / \mathrm{O}_{2}$ PEMFC operating at $T=80{ }^{\circ} \mathrm{C}$ [203]. To reduce the adverse effects of flooding, Yang et al. prepared a series of doubly-pyrolysed MOF-derived FeN-C catalysts, which differ by the pyrolysis temperature used [208]. The most robust catalyst was obtained after a first pyrolysis at $T=1150^{\circ} \mathrm{C}$ (the highest pyrolysis temperature employed in this work) and featured the lowest concentration of nitrogen and oxygen atoms at its surface, i.e. it was the more hydrophobic.

In contrast to mild COR, partially reversible, prolonged $\mathrm{COR}$ leads to $\mathrm{CO}_{2}$ evolution, and as a result, to the destruction of Metal-N-C site. Using identical-location transmission electron microscopy, Choi et al. reported thinning of carbon particles after potential sweeping between 1.2 and 1.5 V vs. RHE at $T=50^{\circ} \mathrm{C}$ in Ar-saturated $0.1 \mathrm{M} \mathrm{HClO}_{4}$ [98]. On line inductively-coupled plasma mass spectrometry and differential electrochemical mass spectrometry (ICP-MS and DEMS, respectively) experiments supported the conclusions, and X-EDS analyses revealed pronounced demetallation in these experimental conditions $[98,115]$. Ultimately, the porous structure of Metal-N-C catalysts collapses, thus restricting the transport of gases to the active 
sites. For example, during the ORR, the cathodic catalytic layer becomes starved from $\mathrm{O}_{2}$ leading to a drop in performance at high current density [209].

\subsection{Effect of hydrogen peroxide}

$\mathrm{H}_{2} \mathrm{O}_{2}$ is the only stable intermediate or by-product of the ORR in acid medium and particularly stresses Metal-N-C catalysts [210]. Indeed, Fenton reactions between transition metal cations and $\mathrm{H}_{2} \mathrm{O}_{2}$ lead to the formation of reactive oxygen species (ROS). $\mathrm{Fe}(\mathrm{III})$ cations are known to produce ROS species, however $\mathrm{Cu}(\mathrm{II})>\mathrm{Cr}(\mathrm{III}) \sim \mathrm{Co}(\mathrm{II})$ are much more active towards $\mathrm{ROS}$ production at $\mathrm{pH} 7$, underlining the importance of this degradation mechanism [211]. Nitrone spin-trap coupled to electron paramagnetic resonance (EPR) experiments of Choi et al. evidenced that ROS form during a 2-hour exposure of Fe-N-C to a 5 wt. $\% \mathrm{H}_{2} \mathrm{O}_{2}$ solution at open circuit potential [112]. Post-treatment XAS and Mössbauer analyses revealed intact Fe- $\mathrm{N}_{\mathrm{x}}$ core structure, but a modification of the second coordination sphere of Fe. Moreover, XPS analyses suggested that the surface of the carbon matrix was chemically oxidized during the $\mathrm{H}_{2} \mathrm{O}_{2}$-treatment and the introduction of the oxygen-groups resulted in an acidification of the surface, modified potential of zero-charge, and increased work function. Oxidation of the carbon surface leads to the depletion from $\pi$-electrons and to a decrease in the TOF of the Fe- $\mathrm{N}_{\mathrm{x}}$ sites. RRDE experiments of Choi et al. reported that $32 \%$ of $\mathrm{O}_{2}$ molecules are transformed into $\mathrm{H}_{2} \mathrm{O}_{2}$ for a thin cathode (Fe-N-C catalyst loading of $100 \mu \mathrm{g} \mathrm{cm}^{-2}$ geo) [130]. In contrast, only $6 \% \mathrm{H}_{2} \mathrm{O}_{2}$ was detected for a thicker cathode (catalyst loading of $800 \mu \mathrm{g} \mathrm{cm}^{-2}$ geo), most likely because the produced $\mathrm{H}_{2} \mathrm{O}_{2}$ molecules decomposed within the thick electrode and were not detected at the ring, as inferred in Section 13.4.1. Similar to the effect of bulk COR, chemical oxidation of the carbon matrix leads to (i) decreased TOF values [112,212], (ii) increased hydrophilic character, and associated flooding issues [203,208,213]. For example, Goellner et al. reported that the ORR activity of Fe-N-C and Co-N-C catalysts is divided by 6 and 3 after ex situ $\mathrm{H}_{2} \mathrm{O}_{2}$ treatment, respectively [212]. Increase of the $\mathrm{H}_{2} \mathrm{O}_{2}$ concentration [210], longer duration of exposure to $\mathrm{H}_{2} \mathrm{O}_{2}$ solution $[83,214]$ and higher temperature of the $\mathrm{H}_{2} \mathrm{O}_{2}$ solution $\left(T \geq 50^{\circ} \mathrm{C}\right)$ [112] accelerate the extent of degradation of the Metal-N-C catalysts. Choi et al. also reported that the ORR selectivity changed from $4 \mathrm{e}^{-}\left(\mathrm{O}_{2}\right.$ molecules to water) to $2 \mathrm{e}^{-} \mathrm{ORR}$ on $\mathrm{H}_{2} \mathrm{O}_{2}$-treated $\mathrm{Fe}-\mathrm{N}-\mathrm{C}$ catalysts in acidic medium, showing that the effect of $\mathrm{H}_{2} \mathrm{O}_{2}$ is autocatalytic. In contrast, exposing the same Fe-N-C catalyst to $\mathrm{H}_{2} \mathrm{O}_{2}$ in alkaline electrolyte does not change the ORR activity nor 
selectivity, as revealed by combined RRDE and spin-trap measurements. Importantly, as the effect of $\mathrm{H}_{2} \mathrm{O}_{2}$ mostly relates to formation of oxygen-containing surface groups onto the carbon surface, it is partially reversible. Indeed, both the ORR activity and the selectivity of the Fe-N$\mathrm{C}$ catalyst could be recovered after electrochemical reduction of the oxygen-containing groups present on the surface of the carbon matrix [112].

\subsection{Migration and aggregation of SMA}

Recently, Kumar et al. used Cs-corrected single-atom resolved STEM to investigate the mobility of Fe single-atoms in Fe-N-C materials [215]. The authors synthesized two Fe-N-C materials: both were first pyrolysed at $T=975{ }^{\circ} \mathrm{C}$ in $\mathrm{N}_{2}: \mathrm{H}_{2}$ atmosphere (93:7), and one encountered a second pyrolysis at $T=950{ }^{\circ} \mathrm{C}$ in $\mathrm{N}_{2}: \mathrm{NH}_{3}$ atmosphere (90:10). A secondary pyrolysis usually reveals beneficial to the initial ORR activity, more particularly when the material is pyrolysed under $\mathrm{NH}_{3}[103,216]$; however this effect is known to be non-durable $[11,13,217]$. Kumar et al. reported that $\mathrm{Fe}$ atoms are mobile and cluster on the doubly-pyrolysed $\mathrm{Fe}-\mathrm{N}-\mathrm{C}$ material, not on the Fe-N-C material submitted to a single pyrolysis after square-wave potential cycling between 0.6 and $1.0 \mathrm{~V} v s$. RHE in Ar-saturated $0.1 \mathrm{M} \mathrm{H}_{2} \mathrm{SO}_{4}$ thermostated at $80^{\circ} \mathrm{C}$. Raman spectroscopy results suggested that the carbon matrix of the doubly-pyrolysed material was more prone to COR than that of singly-pyrolysed material, thus facilitating the movement of the SMA in the oxidative potential range investigated in this study.

\subsection{Combined effects}

Thanks to well-designed ASTs, degradation mechanisms and the associated effects on electrocatalytic reactions have been isolated. However, several stressors are present during the life of SMAC in the operating conditions of an electrochemical device, and a combination of stressors may influence or even revert the predominant degradation mechanism. Perhaps the most striking example of this assertion was provided by Kumar et al. [127]. The authors assessed the long-term ORR activity of a MOF-derived Fe-N-C SMAC using the same AST (10,000 squarewave potential steps between 0.6 and $1.0 \mathrm{~V} v s$. RHE $-3 \mathrm{~s}$ at each potential $-T=80{ }^{\circ} \mathrm{C}$ ) in Arsaturated or $\mathrm{O}_{2}$-saturated $0.1 \mathrm{M} \mathrm{H}_{2} \mathrm{SO}_{4}$. Four times higher ORR activity loss were assessed when performing load cycling AST in $\mathrm{O}_{2}-v s$. Ar-saturated electrolyte. Combined results from cyclic voltammetry, nitrite stripping, X-ray absorption, ${ }^{57} \mathrm{Fe}$ Mössbauer, Raman and X-EDS 
spectroscopies revealed that the more pronounced drop in ORR activity was due to ROS-induced carbon corrosion, and associated formation of nanometric Fe-oxide particles upon reprecipitation of $\mathrm{Fe}$ cations leached from $\mathrm{Fe}-\mathrm{N}_{\mathrm{x}}$ sites. Recently, Osmieri et al. also reported that small Fe clusters form after a $100 \mathrm{~h}$ ageing test in potentiostatic conditions $(U=0.85 \mathrm{~V}$ under air), and a loss of $70 \%$ of the initial ORR activity was monitored [218]. EELS analyses revealed that single $\mathrm{Fe}$ atoms were not coordinated with nitrogen anymore hence they became mobile on the carbon support. In contrast, the same ageing test performed under inert atmosphere had little to no effect. The pivotal role of $\mathrm{O}_{2}$, in combination with electrochemical potential and acidic $\mathrm{pH}$, was also underlined in the operando Mossbauer study of Li et al. [123]. Using two Fe-N$\mathrm{C}$ catalysts, pyrolysed either in $\mathrm{Ar}$ or $\mathrm{NH}_{3}$ atmosphere, the authors provided evidence that SMAC initially comprising two distinct Fe- $\mathrm{N}_{\mathrm{x}}$ sites (S1 and S2) degrade via the transformation of S1 into Fe nano-oxides while the structure and number of S2 were unmodified after $50 \mathrm{~h}$ operation in a PEMFC cathode running on $\mathrm{H}_{2} / \mathrm{O}_{2}$. These findings show that the main reason for the loss of performance of Fe-N-C catalysts at a PEMFC cathode is a ROS-triggered C corrosion, yielding Fe-N 4 site demetallation and reprecipitation, in agreement with former results of Chenitz et al. [219].

\section{Summary and conclusions}

Notable progress has been achieved from the early works of Jasinski highlighting the potential use of $3 \mathrm{~d}$ transition metal macrocyclic complexes as fuel cell electrocatalysts, to the structurally diverse, highly active, Metal-N-C electrocatalysts from today. The existence of Metal- $\mathrm{N}_{\mathrm{x}}$ sites covalently embedded in the carbon matrix for pyrolysed Metal-N-C materials is now well established and recognized in the material's field, with a first coordination structure bearing some analogy with that of Metal-N4 macrocycles. Such Metal-N-C materials have however key advantages over non-pyrolysed macrocycles, such as enhanced stability and activity, and enhanced electron conductivity. The methods to synthesize the Metal-N-C are diverse, ranging from the functionalization of carbon black with nitrogen and metal-based precursors to approaches entirely foregoing the use of a pre-existing carbon support, instead combining carbon, nitrogen- and metal-precursors with different approaches such as hard-template (e.g. with silica) and soft-template approaches (e.g. metal-organic-framework, polymers, etc.). Although the doping with $\mathrm{Fe}$ and Co have hitherto been most often studied, it has also been recently shown 
that most of the $3 \mathrm{~d}$ transition metals and also other elements (e.g. tin, platinum-group metals, etc.) can be integrated as SMA in nitrogen-doped carbon materials. Progresses of Metal-Ncarbon SMAC are not limited to the optimization and further development of the syntheses processes, but also evolve around improved understanding of the chemistry, structure and reactivity of those materials. A broad range of Metal- $\mathrm{N}_{\mathrm{x}}$ moieties can be observed, with the detailed structure, proportion and site density driven by the synthesis process, including nitrogenbased groups and metal, nitrogen-containing sites. The latter are of critical importance. Their structure is now partially understood, owing to a wide range of analytical tools, including Mössbauer and X-ray absorption spectroscopy, electrochemistry and microscopy, which provide insights on their direct environment and electronic structure.

The Metal-N-C SMAC exhibit interesting electrocatalytic properties for the ORR, the $\mathrm{H}_{2} \mathrm{O}_{2}$ generation and the $\mathrm{CO}_{2} \mathrm{RR}$. In acidic environment, the $\mathrm{Fe}-\mathrm{N}_{4}$ sites are able to catalyse the $4 \mathrm{e}^{-}$ exchange required to transform $\mathrm{O}_{2}$ in water. Their activity can be tuned by modifying the degree of graphitization and disorder (i.e. the functionalization, density of nitrogen, oxygen and moieties, etc.) of the carbon basal plane the metallic atoms are embedded into. As for the $\mathrm{CO}_{2} \mathrm{RR}$, they do exhibit a wide range of selectivity for $\mathrm{CO}$ generation, ranging from ca $25 \%$ on Co-N$\mathrm{C}$ to near $100 \%$ on Ni-N-C, thus allowing the direct production of syngas (a 1:2 - 1:3 CO: $\mathrm{H}_{2}$ mixture) when using Metal-N-C electrocatalysts. Other value-added products can be synthesized through $\mathrm{CO}_{2} \mathrm{RR}$, e.g. formate by using Sn-N-C or ethanol by using $\mathrm{Cu}-\mathrm{N}-\mathrm{C}$. For the latter, it was shown that ethanol production resulted from the dynamic clustering of $\mathrm{Cu}-\mathrm{N}_{\mathrm{x}}$ sites in operando. Structural changes during electrocatalysis are not uncommon, and Metal-N-C catalysts can undergo various degradation processes when used as ORR cathode catalysts, including not only formation of clusters, but also (i) carbon corrosion, which incidentally leads to the destruction of the active sites; (ii) demetallation, i.e. the replacement of the metal cation by protons in the Metal-N 4 moiety and (iii) protonation of the nitrogen groups and (iv) the partial (reversible) oxidation of the carbon basal plane, which impacts the electron density at Metal$\mathrm{N}_{\mathrm{x}}$ sites and thus their TOF towards the ORR.

This chapter aimed to provide a comprehensive overview of the different aspects of Metal-N$\mathrm{C}$ electrocatalysts, including their synthetic processes, structures, activity for various electrochemical reactions and durability; to highlight the notable progresses achieved throughout the 
past decade, but also to hint at the remaining challenges. Further investigation into the structural properties of Metal-N-C electrocatalysts and, more specifically, of the still-debated exact structure and electronic state of the active sites, will require techniques that specifically probe such moieties, e.g. advanced microscopic techniques or other local techniques. Most approaches to assess the active site structure in Metal-N-C SMAC nowadays heavily rely on theoretical models to interpret and/or fit the experimental data, while experimental data are most often collected on a high number of SMA (even for techniques regarded as probing a small surface area, such as XAS). Metal-N-C's recent venture in new reactions pastures was a success, as they showed promise as $\mathrm{CO}_{2}$ to $\mathrm{CO}$ or $\mathrm{CO}_{2}$ to formate electrocatalysts, but those paths can further be explored (i) in $\mathrm{CO}_{2} \mathrm{RR}$, by enabling the Metal-N-C to synthesize value-added-products 'beyond' $\mathrm{CO}$, i.e. $\mathrm{C}_{2}$ species either through the design of dual-sites or cascade catalysts; (ii) in other reactions, such as nitrate reduction, by investigating the potentiality of those materials, hinted by the recent results obtained for the nitrogen reduction reaction. Finally, the Metal-N-C electrocatalysts were originally thought as an alternative for platinum-based electrocatalysts in proton exchange membrane fuel cells. Hence, the most important challenge was, and remain, to close the activity gap that exist between those two families, along with going forward in implementing Metal-N-C in actual devices to achieve, in the upcoming years, a successful implementation in noble-metal-free fuel cell vehicles. 


\section{7. $\quad$ References}

1. Lim, T., Jung, G.Y., Kim, J.H., et al. (2020) Atomically dispersed Pt-N $\mathrm{N}_{4}$ sites as efficient and selective electrocatalysts for the chlorine evolution reaction. Nature Communications, 11: 412.

2. Liu, D., Li, X., Chen, S., et al. (2019) Atomically dispersed platinum supported on curved carbon supports for efficient electrocatalytic hydrogen evolution. Nature Energy, 4 (6): 512-518.

3. Wang, J., Tan, H.-Y., Kuo, T.-R., et al. (2021) In situ identifying the dynamic structure behind activity of atomically dispersed platinum catalyst toward hydrogen evolution reaction. Small, 17 (16): 2005713.

4. Chao, T., Luo, X., Chen, W., et al. (2017) Atomically dispersed copper-platinum dual sites alloyed with palladium nanorings catalyze the hydrogen evolution reaction. Angewandte Chemie - International Edition, 56 (50): 16047-16051.

5. G. Rinaldo, S., Stumper, J., Eikerling, M. (2010) Physical theory of platinum nanoparticle dissolution in polymer electrolyte fuel cells. The Journal of Physical Chemistry C, 114 (13): $5773-5785$.

6. Darling, R.M., Meyers, J.P. (2003) Kinetic model of platinum dissolution in PEMFCs. Journal of The Electrochemical Society, 150 (11): A1523.

7. Calle-Vallejo, F., Martínez, J.I., García-Lastra, J.M., et al. (2014) Fast prediction of adsorption properties for platinum nanocatalysts with generalized coordination numbers. Angewandte Chemie - International Edition, 53 (32): 8316-8319.

8. Jasinski, R. (1964) A new fuel cell cathode catalyst. Nature, 201: 1212-1213.

9. Jasinski, R. (1965) Cobalt phthalocyanine as a fuel cell cathode. Journal of the Electrochemical Society, 112 (5): 526-528.

10. Gupta, S., Tryk, D., Bae, I., et al. (1989) Heat-treated polyacrylonitrile-based catalysts for oxygen electroreduction. Journal of Applied Electrochemistry, 19 (1): 19-27.

11. Lefèvre, M., Proietti, E., Jaouen, F., et al. (2009) Iron-based catalysts with improved oxygen reduction activity in polymer electrolyte fuel cells. Science, 324 (5923): 71-74.

12. Wu, G., More, K.L., Johnston, C.M., et al. (2011) High-performance electrocatalysts for oxygen reduction derived from polyaniline, iron, and cobalt. Science, 332 (6028): 443-447.

13. Proietti, E., Jaouen, F., Lefèvre, M., et al. (2011) Iron-based cathode catalyst with enhanced power density in polymer electrolyte membrane fuel cells. Nature Communications, 2: 416.

14. Ma, S., Goenaga, G.A., Call, A. V, et al. (2011) Cobalt imidazolate framework as precursor for oxygen reduction reaction electrocatalysts. Chemistry - A European Journal, 17 (7): 2063-2067.

15. Jahnke, H., Schönborn, M., Zimmermann, G. (1976) Organic dyestuffs as catalysts for fuel cells. Topics in Current Chemistry, 61: 133-181. 
16. K. Radyushkina, R.H. Burshtein, B.D. Berezin, M.R. Tarasevich, S.L. (1973) in Elektrokhimiya translated as "Soviet Electrochemistry," 9: 410.

17. Blomquist, J., Moberg, L.C., Johansson, L.Y., et al. (1981) Mössbauer measurements on iron phthalocyanines. Journal of Inorganic and Nuclear Chemistry, 43 (10): 2287-2292.

18. Dodelet, J.-P. (2006) Oxygen reduction in PEM fuel cell conditions: Heat-treated non-precious metal- $\mathrm{N}_{4}$ macrocycles and beyond, in $N_{4}$-Macrocyclic Metal Complexes (eds. Zagal, J., Bedioui, F., and Dodelet, J.-P.), Springer Science, New York, 83-147.

19. Scherson, D.A., Yao, S.B., Yeager, E.B., et al. (1983) In situ and ex situ Moessbauer spectroscopy studies of iron phthalocyanine adsorbed on high surface area carbon. The Journal of Physical Chemistry, 87 (6): 932-943.

20. Mineva, T., Matanovic, I., Atanassov, P., et al. (2019) Understanding active sites in pyrolyzed Fe-N-C catalysts for fuel cell cathodes by bridging density functional theory calculations and ${ }^{57} \mathrm{Fe}$ Mössbauer spectroscopy. ACS Catalysis, 9 (10): 9359-9371.

21. van Veen, J.A.R., Visser, C. (1979) Oxygen reduction on monomeric transition metal phthalocyanines in acid electrolyte. Electrochimica Acta, 24 (9): 921-928.

22. Wiesener, K., Ohms, D., Neumann, V., et al. (1989) $\mathrm{N}_{4}$ macrocycles as electrocatalysts for the cathodic reduction of oxygen. Materials Chemistry and Physics, 22 (3): 457-475.

23. van Veen, J.A.R., Colijn, H.A. (1981) Oxygen reduction on transition-metal porphyrins in acid electrolyte II. Stability. Berichte der Bunsengesellschaft für physikalische Chemie, 85 (9): 700 704.

24. Faubert, G., Lalande, G., Côté, R., et al. (1996) Heat-treated iron and cobalt tetraphenylporphyrins adsorbed on carbon black: physical characterization and catalytic properties of these materials for the reduction of oxygen in polymer electrolyte fuel cells. Electrochimica Acta, 41: 1689-1701.

25. Bagotzky, V.S., Tarasevich, M.R., Radyushkina, K.A., et al. (1978) Electrocatalysis of the oxygen reduction process on metal chelates in acid electrolyte. Journal of Power Sources, 2 (3): 233-240.

26. Gouérec, P., Biloul, A., Contamin, O., et al. (1997) Oxygen reduction in acid media catalyzed by heat treated cobalt tetraazaannulene supported on an active charcoal: correlations between the performances after longevity tests and the active site configuration as seen by XPS and ToFSIMS. Journal of Electroanalytical Chemistry, 422 (1): 61-75.

27. Vallejos-Burgos, F., Utsumi, S., Hattori, Y., et al. (2012) Pyrolyzed phthalocyanines as surrogate carbon catalysts: Initial insights into oxygen-transfer mechanisms. Fuel, 99: 106-117.

28. van Wingerden, B., van Veen, J.A.R., Mensch, C.T.J. (1988) An extended X-ray absorption fine structure study of heat-treated cobalt porphyrin catalysts supported on active carbon. Journal of 
the Chemical Society, Faraday Transactions 1: Physical Chemistry in Condensed Phases, 84 (1): 65-74.

29. Schulenburg, H., Stankov, S., Schünemann, V., et al. (2003) Catalysts for the oxygen reduction from heat-treated iron(III) tetramethoxyphenylporphyrin chloride: Structure and stability and active sites. The Journal of Physical Chemistry B, 107 (34): 9034-9041.

30. Lefèvre, M., Dodelet, J.P., Bertrand, P. (2002) Molecular oxygen reduction in PEM fuel cells: Evidence for the simultaneous presence of two active sites in Fe-based catalysts. The Journal of Physical Chemistry B, 106 (34): 8705-8713.

31. Lefèvre, M., Dodelet, J.P., Bertrand, P. (2005) Molecular oxygen reduction in PEM fuel cell conditions: ToF-SIMS analysis of Co-based electrocatalysts. The Journal of Physical Chemistry $B, 109$ (35): 16718-16724.

32. Tributsch, H., Koslowski, U.I., Dorbandt, I. (2008) Experimental and theoretical modeling of Fe-, Co-, Cu-, Mn-based electrocatalysts for oxygen reduction. Electrochimica Acta, 53 (5): 2198-2209.

33. Chang, S.-T., Wang, C.-H., Du, H.-Y., et al. (2012) Vitalizing fuel cells with vitamins: pyrolyzed vitamin B12 as a non-precious catalyst for enhanced oxygen reduction reaction of polymer electrolyte fuel cells. Energy and Environmental Science, 5 (1): 5305-5314.

34. Bouwkamp-Wijnoltz, A.L., Visscher, W., van Veen, J.A.R., et al. (2002) On active-site heterogeneity in pyrolyzed carbon-supported iron porphyrin catalysts for the electrochemical reduction of oxygen : an in situ Mössbauer study. The Journal of Physical Chemistry B., 106 (50): 12993-13001.

35. Ziegelbauer, J.M., Olson, T.S., Pylypenko, S., et al. (2008) Direct spectroscopic observation of the structural origin of peroxide generation from Co-based pyrolyzed porphyrins for ORR applications. The Journal of Physical Chemistry C, 112 (24): 8839-8849.

36. Kramm, U.I., Zana, A., Vosch, T., et al. (2016) On the structural composition and stability of $\mathrm{Fe}-\mathrm{N}-\mathrm{C}$ catalysts prepared by an intermediate acid leaching. Journal of Solid State Electrochemistry, 20 (4): 969-981.

37. Herrmann, I., Kramm, U.I., Fiechter, S., et al. (2009) Oxalate supported pyrolysis of CoTMPP as electrocatalysts for the oxygen reduction reaction. Electrochimica Acta, 54 (18): 4275-4287.

38. Kramm, U.I., Herrmann-Geppert, I., Behrends, J., et al. (2016) On an easy way to prepare metalnitrogen doped carbon with exclusive presence of $\mathrm{MeN}_{4}$-type sites active for the ORR. Journal of the American Chemical Society, 138 (2): 635-640.

39. Liberman, I., Shimoni, R., Ifraemov, R., et al. (2020) Active-site modulation in an Fe-porphyrinbased metal-organic framework through ligand axial coordination: Accelerating electrocatalysis and charge-transport kinetics. Journal of the American Chemical Society, 142 (4): 1933-1940. 
40. Lions, M., Tommasino, J.-B., Chattot, R., et al. (2017) Insights into the mechanism of electrocatalysis of the oxygen reduction reaction by a porphyrinic metal organic framework. Chemical Communications, 53 (48): 6496-6499.

41. Cheon, J.Y., Kim, T., Choi, Y., et al. (2013) Ordered mesoporous porphyrinic carbons with very high electrocatalytic activity for the oxygen reduction reaction. Scientific Reports, 3 (1): 2715.

42. Zion, N., Douglin, J.C., Cullen, D.A., et al. (2021) Porphyrin aerogel catalysts for oxygen reduction reaction in anion-exchange membrane fuel cells. Advanced Functional Materials, 31 (24): 2100963.

43. Bron, M., Radnik, J., Fieber-Erdmann, M., et al. (2002) EXAFS, XPS and electrochemical studies on oxygen reduction catalysts obtained by heat treatment of iron phenanthroline complexes supported on high surface area carbon black. Journal of Electroanalytical Chemistry, 535 (1): 113-119.

44. Jaouen, F., Marcotte, S., Dodelet, J.P., et al. (2003) Oxygen reduction catalysts for polymer electrolyte fuel cells from the pyrolysis of iron acetate adsorbed on various carbon supports. The Journal of Physical Chemistry B, 107 (6): 1376-1386.

45. Villers, D., Jacques-Bédard, X., Dodelet, J.-P. (2004) Fe-based catalysts for oxygen reduction in PEM fuel cells. Journal of The Electrochemical Society, 151 (9): A1507.

46. Lefèvre, M., Dodelet, J.P. (2008) Fe-based electrocatalysts made with microporous pristine carbon black supports for the reduction of oxygen in PEM fuel cells. Electrochimica Acta, $\mathbf{5 3}$ (28): 8269-8276.

47. Jaouen, F., Charreteur, F., Dodelet, J.P. (2006) Fe-based catalysts for oxygen reduction in PEMFCs. Journal of The Electrochemical Society, 153 (4): A689.

48. Charreteur, F., Jaouen, F., Ruggeri, S., et al. (2008) Fe/N/C non-precious catalysts for PEM fuel cells: Influence of the structural parameters of pristine commercial carbon blacks on their activity for oxygen reduction. Electrochimica Acta, 53 (6): 2925-2938.

49. Charreteur, F., Ruggeri, S., Jaouen, F., et al. (2008) Increasing the activity of Fe/N/C catalysts in PEM fuel cell cathodes using carbon blacks with a high-disordered carbon content. Electrochimica Acta, 53 (23): 6881-6889.

50. Jaouen, F., Dodelet, J.-P. (2007) Average turn-over frequency of $\mathrm{O}_{2}$ electro-reduction for $\mathrm{Fe} / \mathrm{N} / \mathrm{C}$ and Co/N/C catalysts in PEFCs. Electrochimica Acta, 52 (19): 5975-5984.

51. Ratso, S., Ranjbar Sahraie, N., Sougrati, M.T., et al. (2018) Synthesis of highly-active Fe-N-C catalysts for PEMFC with carbide-derived carbons. Journal of Materials Chemistry A, 6 (30): 14663-14674.

52. Ratso, S., Kruusenberg, I., Käärik, M., et al. (2018) Highly efficient transition metal and nitrogen co-doped carbide-derived carbon electrocatalysts for anion exchange membrane fuel cells. 
Journal of Power Sources, 375: 233-243.

53. Lefèvre, M., Proietti, E., Jaouen, F., et al. (2009) Iron-based catalysts for oxygen reduction in PEM fuel cells: Expanded study using the pore-filling method. ECS Transactions, 25: 105-115.

54. Pylypenko, S., Mukherjee, S., Olson, T.S., et al. (2008) Non-platinum oxygen reduction electrocatalysts based on pyrolyzed transition metal macrocycles. Electrochimica Acta, 53: $7875-7883$.

55. Gokhale, R., Chen, Y., Serov, A., et al. (2017) Novel dual templating approach for preparation of highly active Fe-N-C electrocatalyst for oxygen reduction. Electrochimica Acta, 224: 49-55.

56. Serov, A., Artyushkova, K., Niangar, E., et al. (2015) Nano-structured non-platinum catalysts for automotive fuel cell application. Nano Energy, 16: 293-300.

57. Serov, A., Robson, M.H., Halevi, B., et al. (2012) Highly active and durable templated non-PGM cathode catalysts derived from iron and aminoantipyrine. Electrochemistry Communications, 22: $53-56$.

58. Liu, G., Li, X., Ganesan, P., et al. (2009) Development of non-precious metal oxygen-reduction catalysts for PEM fuel cells based on N-doped ordered porous carbon. Applied Catalysis B: Environmental, 93 (1): 156-165.

59. Primbs, M., Sun, Y., Roy, A., et al. (2020) Establishing reactivity descriptors for platinum group metal (PGM)-free Fe-N-C catalysts for PEM fuel cells. Energy and Environmental Science, 13 (8): 2480-2500.

60. Monteverde Videla, A.H.A., Osmieri, L., Armandi, M., et al. (2015) Varying the morphology of $\mathrm{Fe}-\mathrm{N}-\mathrm{C}$ electrocatalysts by templating iron phthalocyanine precursor with different porous $\mathrm{SiO}_{2}$ to promote the oxygen reduction reaction. Electrochimica Acta, 177: 43-50.

61. Sa, Y.J., Seo, D.-J., Woo, J., et al. (2016) A general approach to preferential formation of active $\mathrm{Fe}-\mathrm{N}_{\mathrm{x}}$ sites in $\mathrm{Fe}-\mathrm{N} / \mathrm{C}$ electrocatalysts for efficient oxygen reduction reaction. Journal of the American Chemical Society, 138 (45): 15046-15056.

62. Jaouen, F., Lefèvre, M., Dodelet, J.P., et al. (2006) Heat-treated $\mathrm{Fe} / \mathrm{N} / \mathrm{C}$ catalysts for $\mathrm{O}_{2}$ electroreduction: Are active sites hosted in micropores? The Journal of Physical Chemistry B, 110 (11): 5553-5558.

63. Barkholtz, H.M., Liu, D.-J. (2017) Advancements in rationally designed PGM-free fuel cell catalysts derived from metal-organic frameworks. Materials Horizons, 4 (1): 20-37.

64. Morozan, A., and Jaouen, F. (2012) Metal organic frameworks for electrochemical applications. Energy and Environmental Science, 5 (11): 9269-9290.

65. Xia, W., Zhu, J., Guo, W., et al. (2014) Well-defined carbon polyhedrons prepared from nano metal-organic frameworks for oxygen reduction. Journal of Materials Chemistry A, 2 (30): 11606-11613. 
66. Zhang, H., Hwang, S., Wang, M., et al. (2017) Single atomic iron catalysts for oxygen reduction in acidic media: Particle size control and thermal activation. Journal of the American Chemical Society, 139 (40): 14143-14149.

67. Armel, V., Hannauer, J., Jaouen, F. (2015) Effect of ZIF-8 crystal size on the $\mathrm{O}_{2}$ electro-reduction performance of pyrolyzed Fe-N-C catalysts. Catalysts, 5: 1333-1351.

68. Wang, X., Zhang, H., Lin, H., et al. (2016) Directly converting Fe-doped metal-organic frameworks into highly active and stable Fe-N-C catalysts for oxygen reduction in acid. Nano Energy, 25: 110-119.

69. Zhao, D., Shui, J.-L., Grabstanowicz, L.R., et al. (2014) Highly efficient non-precious metal electrocatalysts prepared from one-pot synthesized zeolitic imidazolate frameworks. Advanced Materials, 26: 1093-1097.

70. Wang, J., Han, G., Wang, L., et al. (2018) ZIF-8 with ferrocene encapsulated: A promising precursor to single-atom $\mathrm{Fe}$ embedded nitrogen-doped carbon as highly efficient catalyst for oxygen electroreduction. Small, 14 (15): 1704282.

71. Palaniselvam, T., Biswal, B.P., Banerjee, R., et al. (2013) Zeolitic imidazolate framework (ZIF)derived, hollow-core, nitrogen-doped carbon nanostructures for oxygen-reduction reactions in PEFCs. Chemistry - A European Journal, 19 (28): 9335-9342.

72. Armel, V., Hindocha, S., Salles, F., et al. (2017) Structural descriptors of zeolitic-imidazolate frameworks are keys to the activity of Fe-N-C catalysts. Journal of the American Chemical Society, 139 (1): 453-464.

73. Zitolo, A., Goellner, V., Armel, V., et al. (2015) Identification of catalytic sites for oxygen reduction in iron- and nitrogen-doped graphene materials. Nature materials, 14 (9): 937-942.

74. He, Y., Hwang, S., Cullen, D.A., et al. (2019) Highly active atomically dispersed $\mathrm{CoN}_{4}$ fuel cell cathode catalysts derived from surfactant-assisted MOFs: carbon-shell confinement strategy. Energy and Environmental Science, 12 (1): 250-260.

75. Yin, P., Yao, T., Wu, Y., et al. (2016) Single cobalt atoms with precise N-coordination as superior oxygen reduction reaction catalysts. Angewandte Chemie - International Edition, 55 (36): 10800-10805.

76. Liu, S., Wang, M., Yang, X., et al. (2020) Chemical vapor deposition for atomically dispersed and nitrogen coordinated single metal site catalysts. Angewandte Chemie - International Edition, 59 (48): 21698-21705.

77. Jiao, L., Li, J., Richard, L.L., et al. (2021) Chemical vapor deposition of Fe-N-C oxygen reduction catalysts with full utilization of dense Fe- $\mathrm{N}_{4}$ sites. Nature Materials, Just Accep: 10.1038/s41563-021-01030-2.

78. Mehmood, A., Pampel, J., Ali, G., et al. (2018) Facile metal coordination of active site imprinted 
nitrogen doped carbons for the conservative preparation of non-noble metal oxygen reduction electrocatalysts. Advanced Energy Materials, 8 (9): 1701771.

79. Jiang, Z., Yu, J., Huang, T., et al. (2018) Recent advance on polyaniline or polypyrrole-derived electrocatalysts for oxygen reduction reaction. Polymers, 10 (12): 1397.

80. Ghosh, D., Giri, S., Mandal, A., et al. (2013) $\mathrm{H}_{+}, \mathrm{Fe}_{3+}$ codoped polyaniline/MWCNTs nanocomposite: Superior electrode material for supercapacitor application. Applied Surface Science, 276: 120-128.

81. Ferrandon, M., Kropf, A.J., Myers, D.J., et al. (2012) Multitechnique characterization of a polyaniline-iron-carbon oxygen reduction catalyst. The Journal of Physical Chemistry C, 116 (30): 16001-16013.

82. Wu, G., Nelson, M.A., Mack, N.H., et al. (2010) Titanium dioxide-supported non-precious metal oxygen reduction electrocatalyst. Chemical Communications, 46 (40): 7489-7491.

83. Wu, G., Artyushkova, K., Ferrandon, M., et al. (2009) Performance durability of polyanilinederived non-precious cathode catalysts. ECS Transactions, 25: 1299-1311.

84. Cullen, D.A., Chung, H.T., More, K.L., et al. (2017) Direct atomic-level insight into the active sites of a high-performance PGM-free ORR catalyst. Science, 357 (6350): 479-484.

85. Yin, X., Chung, H.T., Martinez, U., et al. (2019) PGM-free ORR catalysts designed by templating PANI-type polymers containing functional groups with high affinity to iron. Journal of The Electrochemical Society, 166 (7): F3240-F3245.

86. Shui, J., Chen, C., Grabstanowicz, L., et al. (2015) Highly efficient nonprecious metal catalyst prepared with metal-organic framework in a continuous carbon nanofibrous network. Proceedings of the National Academy of Sciences, 112 (34): 10629-10634.

87. Li, J., Pršlja, P., Shinagawa, T., et al. (2019) Volcano trend in electrocatalytic $\mathrm{CO}_{2}$ reduction activity over atomically dispersed metal sites on nitrogen-doped carbon. ACS Catalysis, 9 (11): 10426-10439.

88. Ju, W., Bagger, A., Hao, G.P., et al. (2017) Understanding activity and selectivity of metalnitrogen-doped carbon catalysts for electrochemical reduction of $\mathrm{CO}_{2}$. Nature Communications, 8: $1-9$.

89. Varela, A.S., Ranjbar Sahraie, N., Steinberg, J., et al. (2015) Metal-doped nitrogenated carbon as an efficient catalyst for direct $\mathrm{CO}_{2}$ electroreduction to $\mathrm{CO}$ and hydrocarbons. Angewandte Chemie - International Edition, 54 (37): 10758-10762.

90. Luo, E., Zhang, H., Wang, X., et al. (2019) Single-atom $\mathrm{Cr}^{-} \mathrm{N}_{4}$ sites designed for durable oxygen reduction catalysis in acid media. Angewandte Chemie - International Edition, 58 (36): 1246912475.

91. Luo, F., Roy, A., Silvioli, L., et al. (2020) $P$-block single-metal-site tin/nitrogen-doped carbon 
fuel cell cathode catalyst for oxygen reduction reaction. Nature Materials, 19 (11): 1215-1223.

92. Chen, S., Lv, C., Liu, L., et al. (2021) High-temperature treatment to engineer the single-atom Pt coordination environment towards highly efficient hydrogen evolution. Journal of Energy Chemistry, 59: 212-219.

93. Xiong, Y., Dong, J., Huang, Z.-Q., et al. (2020) Single-atom Rh/N-doped carbon electrocatalyst for formic acid oxidation. Nature Nanotechnology, 15 (5): 390-397.

94. Zhang, Z., Chen, Y., Zhou, L., et al. (2019) The simplest construction of single-site catalysts by the synergism of micropore trapping and nitrogen anchoring. Nature Communications, 10 : 1657.

95. Ye, S., Luo, F., Zhang, Q., et al. (2019) Highly stable single Pt atomic sites anchored on anilinestacked graphene for hydrogen evolution reaction. Energy and Environmental Science, 12 (3): $1000-1007$.

96. Choi, C.H., Kim, M., Kwon, H.C., et al. (2016) Tuning selectivity of electrochemical reactions by atomically dispersed platinum catalyst. Nature Communications, 7: 10922.

97. Yan, Q.-Q., Wu, D.-X., Chu, S.-Q., et al. (2019) Reversing the charge transfer between platinum and sulfur-doped carbon support for electrocatalytic hydrogen evolution. Nature Communications, 10: 4977.

98. Choi, C.H., Baldizzone, C., Grote, J.P., et al. (2015) Stability of Fe-N-C catalysts in acidic medium studied by operando spectroscopy. Angewandte Chemie - International Edition, 54 (43): 12753-12757.

99. Choi, C.H., Baldizzone, C., Polymeros, G., et al. (2016) Minimizing operando demetallation of Fe-N-C electrocatalysts in acidic medium. ACS Catalysis, 6 (5): 3136-3146.

100. Huan, T.N., Ranjbar, N., Rousse, G., et al. (2017) Electrochemical reduction of $\mathrm{CO}_{2}$ catalyzed by Fe-N-C materials: A structure-selectivity study. ACS Catalysis, 7: 1520-1525.

101. Artyushkova, K., Serov, A., Rojas-Carbonell, S., et al. (2015) Chemistry of multitudinous active sites for oxygen reduction reaction in transition metal-nitrogen-carbon electrocatalysts. The Journal of Physical Chemistry C, 119 (46): 25917-25928.

102. Zitolo, A., Ranjbar-Sahraie, N., Mineva, T., et al. (2017) Identification of catalytic sites in cobaltnitrogen-carbon materials for the oxygen reduction reaction. Nature Communications, 8: 957.

103. Kramm, U.I., Abs-Wurmbach, I., Herrmann-Geppert, I., et al. (2011) Influence of the electrondensity of $\mathrm{FeN}_{4}$-centers towards the catalytic activity of pyrolyzed FeTMPPCl-based ORRelectrocatalysts. Journal of the Electrochemical Society, 158 (1): B69-B78.

104. Kramm, U.I., Lefèvre, M., Larouche, N., et al. (2014) Correlations between mass activity and physicochemical properties of Fe/N/C catalysts for the ORR in PEM fuel cell via ${ }^{57} \mathrm{Fe}$ Mössbauer spectroscopy and other techniques. Journal of the American Chemical Society, 136 (3): 978-985.

105. Kabir, S., Artyushkova, K., Kiefer, B., et al. (2015) Computational and experimental evidence 
for a new TM-N $3 / C$ moiety family in non-PGM electrocatalysts. Physical Chemistry Chemical Physics, 17 (27): 17785-17789.

106. Kramm, U.I., Herranz, J., Larouche, N., et al. (2012) Structure of the catalytic sites in Fe/N/Ccatalysts for $\mathrm{O}_{2}$-reduction in PEM fuel cells. Physical Chemistry Chemical Physics, 14 (33): 11673-11688.

107. Matanovic, I., Artyushkova, K., Strand, M.B., et al. (2016) Core level shifts of hydrogenated pyridinic and pyrrolic nitrogen in the nitrogen-containing graphene-based electrocatalysts: inplane vs edge defects. The Journal of Physical Chemistry C, 120 (51): 29225-29232.

108. Matanovic, I., Artyushkova, K., Atanassov, P. (2018) Understanding PGM-free catalysts by linking density functional theory calculations and structural analysis: Perspectives and challenges. Current Opinion in Electrochemistry, 9: 137-144.

109. Tylus, U., Jia, Q., Strickland, K., et al. (2014) Elucidating oxygen reduction active sites in pyrolyzed metal-nitrogen coordinated non-precious-metal electrocatalyst systems. The Journal of Physical Chemistry C, 118 (17): 8999-9008.

110. Li, J., Ghoshal, S., Liang, W., et al. (2016) Structural and mechanistic basis for the high activity of Fe-N-C catalysts toward oxygen reduction. Energy and Environmental Science, 9 (7): 24182432.

111. Ramaswamy, N., Tylus, U., Jia, Q., et al. (2013) Activity descriptor identification for oxygen reduction on nonprecious electrocatalysts: Linking surface science to coordination chemistry. Journal of the American Chemical Society, 135 (41): 15443-15449.

112. Choi, C.H., Lim, H., Chon, G., et al. (2018) The Achilles' heel of iron-based catalysts during oxygen reduction in an acidic medium. Energy and Environmental Science, 11 (11): 3176-3182.

113. Martinez, U., Holby, E.F., Babu, S.K., et al. (2019) Experimental and theoretical trends of PGMfree electrocatalysts for the oxygen reduction reaction with different transition metals. Journal of the Electrochemical Society, 166 (7): F3136-F3142.

114. Wan, X., Liu, X., Li, Y., et al. (2019) Fe-N-C electrocatalyst with dense active sites and efficient mass transport for high-performance proton exchange membrane fuel cells. Nature Catalysis,.

115. Kumar, K., Gairola, P., Lions, M., et al. (2018) Physical and chemical considerations for improving catalytic activity and stability of non-precious-metal oxygen reduction reaction catalysts. ACS Catalysis, 8 (12): 11264-11276.

116. Jia, Q., Ramaswamy, N., Tylus, U., et al. (2016) Spectroscopic insights into the nature of active sites in iron-nitrogen-carbon electrocatalysts for oxygen reduction in acid. Nano Energy, 29: $65-82$.

117. Osmieri, L. (2019) Transition metal-nitrogen-carbon (M-N-C) catalysts for oxygen reduction reaction. Insights on synthesis and performance in polymer electrolyte fuel cells. 
ChemEngineering, 3 (1): 16.

118. Sun, Y., Silvioli, L., Sahraie, N.R., et al. (2019) Activity-selectivity trends in the electrochemical production of hydrogen peroxide over single-site metal-nitrogen-carbon catalysts. Journal of the American Chemical Society, 141 (31): 12372-12381.

119. Saveleva, V.A., Ebner, K., Ni, L., et al. (2021) Potential-induced spin changes in $\mathrm{Fe} / \mathrm{N} / \mathrm{C}$ electrocatalysts assessed by in situ X-ray emission spectroscopy. Angewandte Chemie International Edition, 60 (21): 11707-11712.

120. Kramm, U.I., Ni, L., Wagner, S. (2019) ${ }^{57} \mathrm{Fe}$ Mössbauer spectroscopy characterization of electrocatalysts. Advanced Materials, 31 (31): 1805623.

121. Gallenkamp, C., Kramm, U.I., Proppe, J., et al. (2021) Calibration of computational Mössbauer spectroscopy to unravel active sites in FeNC catalysts for the oxygen reduction reaction. International Journal of Quantum Chemistry, 121 (3): 1-19.

122. Ni, L., Gallenkamp, C., Paul, S., et al. (2021) Active site identification in FeNC catalysts and their assignment to the oxygen reduction reaction pathway by in situ ${ }^{57} \mathrm{Fe}$ Mössbauer spectroscopy. Advanced Energy and Sustainability Research, 2 (2): 2000064.

123. Li, J., Sougrati, M.T., Zitolo, A., et al. (2021) Identification of durable and non-durable FeNx sites in Fe-N-C materials for proton exchange membrane fuel cells. Nature Catalysis, 4 (1): 10 19.

124. Kneebone, J.L., Daifuku, S.L., Kehl, J.A., et al. (2017) A combined probe-molecule, Mossbauer, nuclear resonance vibrational spectroscopy, and density functional theory approach for evaluation of potential iron active sites in an oxygen reduction reaction catalyst. The Journal of Physical Chemistry C, 121 (30): 16283-16290.

125. Wagner, S., Auerbach, H., Tait, C.E., et al. (2019) Elucidating the structural composition of an Fe-N-C catalyst by nuclear- and electron-resonance techniques. Angew Chem Int Ed Engl, $\mathbf{5 8}$ (31): 10486-10492.

126. Malko, D., Kucernak, A., Lopes, T. (2016) In situ electrochemical quantification of active sites in Fe-N/C non-precious metal catalysts. Nature Communications, 7: 13285.

127. Kumar, K., Dubau, L., Mermoux, M., et al. (2020) On the influence of oxygen on the degradation of Fe-N-C catalysts. Angew Chem Int Ed Engl, 59 (8): 3235-3243.

128. Sahraie, N.R., Kramm, U.I., Steinberg, J., et al. (2015) Quantifying the density and utilization of active sites in non-precious metal oxygen electroreduction catalysts. Nature Communications, $\mathbf{6}$ : 8618.

129. Bae, G., Kim, H., Choi, H., et al. (2021) Quantification of active site density and turnover frequency: From single-atom metal to nanoparticle electrocatalysts. JACS Au, 1 (5), 586-597.

130. Choi, C.H., Choi, W.S., Kasian, O., et al. (2017) Unraveling the nature of sites active toward 
hydrogen peroxide reduction in Fe-N-C catalysts. Angewandte Chemie - International Edition, 56 (30): 8809-8812.

131. Biddinger, E.J., Deak, D. von, Singh, D., et al. (2011) Examination of catalyst loading effects on the selectivity of $\mathrm{CN}_{\mathrm{x}}$ and Pt/VC ORR catalysts using RRDE. Journal of The Electrochemical Society, 158 (4): B402.

132. Bonakdarpour, A., Lefevre, M., Yang, R., et al. (2008) Impact of loading in RRDE experiments on Fe-N-C catalysts: two- or four-electron oxygen reduction? Electrochemical and Solid-State Letters, 11 (6): B105-B108.

133. Dobrzeniecka, A., Zeradjanin, A., Masa, J., et al. (2013) Application of SECM in tracing of hydrogen peroxide at multicomponent non-noble electrocatalyst films for the oxygen reduction reaction. Catalysis Today, 202 (1): 55-62.

134. Sánchez-Sánchez, C.M., Rodríguez-López, J., Bard, A.J. (2008) Scanning electrochemical microscopy. 60. Quantitative calibration of the SECM substrate generation/tip collection mode and its use for the study of the oxygen reduction mechanism. Analytical Chemistry, 80 (9): 3254 3260 .

135. Dobrzeniecka, A., Zeradjanin, A.R., Masa, J., et al. (2016) Evaluation of kinetic constants on porous, non-noble catalyst layers for oxygen reduction - A comparative study between SECM and hydrodynamic methods. Catalysis Today, 262: 74-81.

136. Jaouen, F., Dodelet, J.-P. (2009) $\mathrm{O}_{2}$ reduction mechanism on non-noble metal catalysts for PEM fuel cells. Part II: A porous-electrode model to predict the quantity of $\mathrm{H}_{2} \mathrm{O}_{2}$ detected by rotating ring-diskelectrode. The Journal of Physical Chemistry C, 113 (34): 15433-15443.

137. Verdaguer-casadevall, A., Deiana, D., Karamad, M., et al. (2014) Trends in the electrochemical synthesis of H2O2. Nano Lett., 14: 1603-1608.

138. Siahrostami, S., Verdaguer-Casadevall, A., Karamad, M., et al. (2013) Enabling direct $\mathrm{H}_{2} \mathrm{O}_{2}$ production through rational electrocatalyst design. Nature Materials, 12 (12): 1137-1143.

139. Jung, E., Shin, H., Lee, B.H., et al. (2020) Atomic-level tuning of Co-N-C catalyst for highperformance electrochemical $\mathrm{H}_{2} \mathrm{O}_{2}$ production. Nature Materials, 19 (4): 436-442.

140. Gao, J., Liu, B. (2020) Progress of electrochemical hydrogen peroxide synthesis over single atom catalysts. ACS Materials Letters, 2 (8): 1008-1024.

141. Gasteiger, H.A., Kocha, S.S., Sompalli, B., et al. (2005) Activity benchmarks and requirements for Pt, Pt-alloy, and non-Pt oxygen reduction catalysts for PEMFCs. Applied Catalysis B: Environmental, 56 (1-2): 9-35.

142. Lacis, A.A., Schmidt, G.A., Rind, D., et al. (2010) Atmospheric $\mathrm{CO}_{2}$ : Principal control knob governing earth's temperature. Science, 330 (6002): 356-359.

143. Guo, S., Asset, T., Atanassov, P. (2021) Catalytic hybrid electrocatalytic/biocatalytic cascades 
for carbon dioxide reduction and valorization. ACS Catalysis, 5172-5188.

144. Bagger, A., Ju, W., Varela, A.S., et al. (2017) Single site porphyrine-like structures advantages over metals for selective electrochemical $\mathrm{CO}_{2}$ reduction. Catalysis Today, 288: 74-78.

145. Pan, F., Deng, W., Justiniano, C., et al. (2018) Identification of champion transition metals centers in metal and nitrogen-codoped carbon catalysts for $\mathrm{CO}_{2}$ reduction. Applied Catalysis B: Environmental, 226: 463-472.

146. Möller, T., Ju, W., Bagger, A., et al. (2019) Efficient $\mathrm{CO}_{2}$ to $\mathrm{CO}$ electrolysis on solid Ni-N-C catalysts at industrial current densities. Energy and Environmental Science, 12: 640-647.

147. Hori, Y., Wakebe, H., Tsukamoto, T., et al. (1994) Electrocatalytic process of CO selectivity in electrochemical reduction of $\mathrm{CO} 2$ at metal electrodes in aqueous media. Electrochimica Acta, 39: $1833-1839$.

148. Bagger, A., Ju, W., Varela, A.S., et al. (2017) Electrochemical $\mathrm{CO}_{2}$ reduction: A classification problem. ChemPhysChem, 18: 3266-3273.

149. Birdja, Y.Y., Pérez-Gallent, E., Figueiredo, M.C., et al. (2019) Advances and challenges in understanding the electrocatalytic conversion of carbon dioxide to fuels. Nature Energy, 4: 732745.

150. Asset, T., Garcia, S.T., Herrera, S., et al. (2019) Investigating the nature of the active sites for the $\mathrm{CO} 2$ reduction reaction on carbon-based electrocatalysts. ACS Catalysis, 9: 7668-7678.

151. Schulz, H. (1999) Short history and present trends of Fischer-Tropsch synthesis. Applied Catalysis A: General, 186: 3-12.

152. Varela, A.S., Ju, W., Strasser, P. (2018) Molecular nitrogen-carbon catalysts, solid metal organic framework catalysts, and solid metal/nitrogen-doped carbon (MNC) catalysts for the electrochemical CO2 reduction. Advanced Energy Materials, 8: 1-35.

153. Pan, Y., Lin, R., Chen, Y., et al. (2018) Design of single-atom Co-N $\mathrm{N}_{5}$ catalytic site: A robust electrocatalyst for $\mathrm{CO}_{2}$ reduction with nearly $100 \% \mathrm{CO}$ selectivity and remarkable stability. Journal of the American Chemical Society, 140: 4218-4221.

154. Sharma, P.P., Wu, J., Yadav, R.M., et al. (2015) Nitrogen-doped carbon nanotube arrays for high-efficiency electrochemical reduction of $\mathrm{CO} 2$ : On the understanding of defects, defect density, and selectivity. Angewandte Chemie - International Edition, 54: 13701-13705.

155. Wu, J., Yadav, R.M., Liu, M., et al. (2015) Achieving highly efficient, selective, and stable $\mathrm{CO}_{2}$ reduction on nitrogen-doped carbon nanotubes. ACS Nano, 9: 5364-5371.

156. Wu, J., Liu, M., Sharma, P.P., et al. (2016) Incorporation of Nitrogen Defects for Efficient Reduction of $\mathrm{CO} 2$ via Two-Electron Pathway on Three-Dimensional Graphene Foam. Nano Letters, 16: 466-470.

157. Ju, W., Bagger, A., Wang, X., et al. (2019) Unraveling Mechanistic Reaction Pathways of the 
Electrochemical CO2 Reduction on Fe-N-C Single-Site Catalysts. ACS Energy Letters, $1663-$ 1671.

158. Varela, A.S., Kroschel, M., Leonard, N.D., et al. (2018) pH effects on the selectivity of the electrocatalytic $\mathrm{CO} 2$ reduction on graphene-embedded Fe-N-C motifs: bridging concepts between molecular homogeneous and solid-state heterogeneous catalysis. ACS Energy Letters, 3: $812-817$.

159. Hu, X.M., Hval, H.H., Bjerglund, E.T., et al. (2018) Selective CO2 reduction to CO in water using Earth-abundant metal and nitrogen-doped carbon electrocatalysts. ACS Catalysis, 8: 62556264.

160. Zhang, C., Yang, S., Wu, J., et al. (2018) Electrochemical CO2 reduction with atomic irondispersed on nitrogen-doped graphene. Advanced Energy Materials, 1703487: 1-9.

161. Pan, F., Zhang, H., Liu, K., et al. (2018) Unveiling active sites of CO2 reduction on nitrogencoordinated and atomically dispersed iron and cobalt catalysts. ACS Catalysis, 8: 3116-3122.

162. Hursán, D., Samu, A.A., Janovák, L., et al. (2019) Morphological attributes govern carbon dioxide reduction on N-doped carbon electrodes. Joule, 3: 1719-1733.

163. Endrődi, B., Kecsenovity, E., Samu, A., et al. (2019) Multilayer electrolyzer stack converts carbon dioxide to gas products at high pressure with high efficiency. ACS Energy Letters, 4 (7): $1770-1777$.

164. Leonard, N., Ju, W., Sinev, I., et al. (2018) The chemical identity, state and structure of catalytically active centers during the electrochemical $\mathrm{CO}_{2}$ reduction on porous Fe-nitrogencarbon (Fe-N-C) materials. Chemical Science, 9: 5064-5073.

165. Karapinar, D., Huan, N.T., Ranjbar Sahraie, N., et al. (2019) Electroreduction of $\mathrm{CO}_{2}$ on singlesite copper-nitrogen-doped carbon material: Selective formation of ethanol and reversible restructuration of the metal sites. Angewandte Chemie - International Edition, 58 (42): 1509815103.

166. Zhao, Y., Liang, J., Wang, C., et al. (2018) Tunable and efficient tin modified nitrogen-doped carbon nanofibers for electrochemical reduction of aqueous carbon dioxide. Advanced Energy Materials, 8 (10): 1-9.

167. Zhang, S., Kang, P., Meyer, T.J. (2014) Nanostructured tin catalysts for selective electrochemical reduction of carbon dioxide to formate. Journal of the American Chemical Society, 136: 1734 1737.

168. Zu, X., Li, X., Liu, W., et al. (2019) Efficient and robust carbon dioxide electroreduction enabled by atomically dispersed $\mathrm{Sn}^{\delta+}$ sites. Advanced Materials, 31 (15): 1-8.

169. Paul, S., Kao, Y.-L., Ni, L., et al. (2021) Influence of the metal center in M-N-C catalysts on the $\mathrm{CO}_{2}$ reduction reaction on gas diffusion electrodes. ACS Catalysis, 11 (9): 5850-5864. 
170. Jia, H.P., Quadrelli, E.A. (2014) Mechanistic aspects of dinitrogen cleavage and hydrogenation to produce ammonia in catalysis and organometallic chemistry: Relevance of metal hydride bonds and dihydrogen. Chemical Society Reviews, 43 (2): 547-564.

171. Seefeldt, L.C., Hoffman, B.M., Dean, D.R. (2009) Mechanism of Mo-dependent nitrogenase. Annual Review of Biochemistry, 78 (1): 701-722.

172. Skúlason, E., Bligaard, T., Gudmundsdóttir, S., et al. (2012) A theoretical evaluation of possible transition metal electro-catalysts for $\mathrm{N}_{2}$ reduction. Physical Chemistry Chemical Physics, 14 (3): $1235-1245$.

173. Han, L., Liu, X., Chen, J., et al. (2019) Atomically dispersed molybdenum catalysts for efficient ambient nitrogen fixation. Angewandte Chemie - International Edition, 58 (8): 2321-2325.

174. Liu, W., Han, L., Wang, H.T., et al. (2020) FeMo sub-nanoclusters/single atoms for neutral ammonia electrosynthesis. Nano Energy, 77: 105078.

175. Wang, M., Liu, S., Qian, T., et al. (2019) Over 56.55\% Faradaic efficiency of ambient ammonia synthesis enabled by positively shifting the reaction potential. Nature Communications, 10: 341 .

176. Suryanto, B.H.R., Du, H.L., Wang, D., et al. (2019) Challenges and prospects in the catalysis of electroreduction of nitrogen to ammonia. Nature Catalysis, 2 (4): 290-296.

177. Choi, J., Suryanto, B.H.R., Wang, D., et al. (2020) Identification and elimination of false positives in electrochemical nitrogen reduction studies. Nature Communications, 11: 1-10.

178. Andersen, S.Z., Čolić, V., Yang, S., et al. (2019) A rigorous electrochemical ammonia synthesis protocol with quantitative isotope measurements. Nature, 570 (7762): 504-508.

179. van Langevelde, P.H., Katsounaros, I., Koper, M.T.M. (2021) Electrocatalytic nitrate reduction for sustainable ammonia production. Joule, 5 (2): 290-294.

180. Shannon, R.D. (1976) Revised effective ionic radii and systematic studies of interatomic distances in halides and chalcogenides. Acta Crystallographica Section A, 32 (5): 751-767.

181. Baranton, S., Coutanceau, C., Roux, C., et al. (2005) Oxygen reduction reaction in acid medium at iron phthalocyanine dispersed on high surface area carbon substrate: tolerance to methanol, stability and kinetics. Journal of Electroanalytical Chemistry, 577 (2): 223-234.

182. Meier, H., Tschirwitz, U., Zimmerhackl, E., et al. (1977) Application of radioisotope techniques for the study of phthalocyanine catalyzed electrochemical processes in fuel cells. The Journal of Physical Chemistry, 81 (8): 712-718.

183. Chen, Z., Jiang, S., Kang, G., et al. (2019) Operando characterization of iron phthalocyanine deactivation during oxygen reduction reaction using electrochemical tip-enhanced Raman spectroscopy. Journal of the American Chemical Society, 141 (39): 15684-15692.

184. Bai, L., Hsu, C.-S., Alexander, D.T.L., et al. (2019) A cobalt-iron double-atom catalyst for the oxygen evolution reaction. Journal of the American Chemical Society, 141 (36): 14190-14199. 
185. Hocking, R.K., Brimblecombe, R., Chang, L.-Y., et al. (2011) Water-oxidation catalysis by manganese in a geochemical-like cycle. Nature Chemistry, 3 (6): 461-466.

186. Kanan, M.W., Nocera, D.G. (2008) In situ formation of an oxygen-evolving catalyst in neutral water containing phosphate and $\mathrm{Co}^{2+}$. Science, 321 (5892): 1072-1075.

187. Anxolabéhère-Mallart, E., Costentin, C., Fournier, M., et al. (2012) Boron-capped tris(glyoximato) cobalt clathrochelate as a precursor for the electrodeposition of nanoparticles catalyzing $\mathrm{H}_{2}$ evolution in water. Journal of the American Chemical Society, 134 (14): 61046107.

188. Ghachtouli, S. El, Guillot, R., Brisset, F., et al. (2013) Cobalt-based particles formed upon electrocatalytic hydrogen production by a cobalt pyridine oxime complex. ChemSusChem, $\mathbf{6}$ (12): 2226-2230.

189. El Ghachtouli, S., Fournier, M., Cherdo, S., et al. (2013) Monometallic cobalt-trisglyoximato complexes as precatalysts for catalytic $\mathrm{H}_{2}$ evolution in water. The Journal of Physical Chemistry C, 117 (33): 17073-17077.

190. Weng, Z., Wu, Y., Wang, M., et al. (2018) Active sites of copper-complex catalytic materials for electrochemical carbon dioxide reduction. Nature Communications, 9: 415.

191. Santori, P.G., Speck, F.D., Li, J., et al. (2019) Effect of pyrolysis atmosphere and electrolyte pH on the oxygen reduction activity, stability and spectroscopic signature of $\mathrm{FeN}_{\mathrm{x}}$ moieties in $\mathrm{Fe}-\mathrm{N}$ C catalysts. Journal of The Electrochemical Society, 166 (7): F3311-F3320.

192. Liu, G., Li, X., Popov, B.N. (2009) Stability study of nitrogen-modified carbon composite catalysts for oxygen reduction reaction in polymer electrolyte membrane fuel cells. ECS Transactions, 25: 1251-1259.

193. Liu, G., Li, X., Lee, J.-W., et al. (2011) A review of the development of nitrogen-modified carbon-based catalysts for oxygen reduction at USC. Catalysis Science and Technology, 1 (2): 207.

194. Herranz, J., Jaouen, F., Lefèvre, M., et al. (2011) Unveiling N-protonation and anion-binding effects on $\mathrm{Fe} / \mathrm{N} / \mathrm{C}$ catalysts for $\mathrm{O}_{2}$ reduction in proton-exchange-membrane fuel cells. The Journal of Physical Chemistry C, 115 (32): 16087-16097.

195. Artyushkova, K., J. Workman, M., Matanovic, I., et al. (2017) Role of surface chemistry on catalyst/ionomer interactions for transition metal-nitrogen-carbon electrocatalysts. ACS Applied Energy Materials, 1 (1): 68-77.

196. Binder, H., Kohling, A., Richter, K., et al. (1964) Über die anodische oxydation von aktivkohlen in wässrigen elektrolyten. Electrochim. Acta, 9: 255.

197. Castanheira, L., Silva, W.O., Lima, F.H.B., et al. (2015) Carbon corrosion in proton-exchange membrane fuel cells: Effect of the carbon structure, the degradation protocol, and the gas 
atmosphere. ACS Catalysis, 2184-2194.

198. Roen, L.M., Paik, C.H., and Jarvi, T.D. (2004) Electrocatalytic corrosion of carbon support in PEMFC cathodes. Electrochemical and Solid-State Letters, 7 (1): A19-A22.

199. Stevens, D.A., Dahn, J.R. (2005) Thermal degradation of the support in carbon-supported platinum electrocatalysts for PEM fuel cells. Carbon, 43 (1): 179-188.

200. Appleby, A.J. (1987) Corrosion in low and high temperature fuel cells - An overview. Corrosion, 43 (7): 398-408.

201. Stevens, D.A., Hicks, M.T., Haugen, G.M., et al. (2005) Ex situ and in situ stability studies of PEMFC catalysts. Journal of the Electrochemical Society, 152 (12): A2309-A2315.

202. Maass, S., Finsterwalder, F., Frank, G., et al. (2008) Carbon support oxidation in PEM fuel cell cathodes. Journal of Power Sources, 176 (2): 444-451.

203. Zhang, G., Chenitz, R., Lefèvre, M., et al. (2016) Is iron involved in the lack of stability of $\mathrm{Fe} / \mathrm{N} / \mathrm{C}$ electrocatalysts used to reduce oxygen at the cathode of PEM fuel cells? Nano Energy, 29: 111-125.

204. Zhang, J., Dai, L. (2015) Heteroatom-doped graphitic carbon catalysts for efficient electrocatalysis of oxygen reduction reaction. ACS Catalysis, 5 (12): 7244-7253.

205. Zhao, S., Li, M., Han, M., et al. (2018) Defect-rich $\mathrm{Ni}_{3} \mathrm{FeN}$ nanocrystals anchored on N-doped graphene for enhanced electrocatalytic oxygen evolution. Advanced Functional Materials, 28 (18): 1706018.

206. Giordano, N., Antonucci, P.L., Passalacqua, E., et al. (1991) Relationship between physicochemical properties and electrooxidation behavior of carbon materials. Electrochimica Acta, 36 (13): 1931-1935.

207. Wang, X.X., Prabhakaran, V., He, Y., et al. (2019) Iron-free cathode catalysts for protonexchange-membrane fuel cells: Cobalt catalysts and the peroxide mitigation approach. Advanced Materials, 31 (31): 1805126.

208. Yang, L., Larouche, N., Chenitz, R., et al. (2015) Activity, performance, and durability for the reduction of oxygen in PEM fuel cells, of Fe/N/C electrocatalysts obtained from the pyrolysis of metal-organic-framework and iron porphyrin precursors. Electrochimica Acta, 159: 184-197.

209. Reshetenko, T., Serov, A., Artyushkova, K., et al. (2016) Tolerance of non-platinum group metals cathodes proton exchange membrane fuel cells to air contaminants. Journal of Power Sources, 324: 556-571.

210. Lefèvre, M., Dodelet, J.P. (2003) Fe-based catalysts for the reduction of oxygen in polymer electrolyte membrane fuel cell conditions: Determination of the amount of peroxide released during electroreduction and its influence on the stability of the catalysts. Electrochimica Acta, 48 (19): 2749-2760. 
211. Strlič, M., Kolar, J., Šelih, V.S., et al. (2003) A comparative study of several transition metals in Fenton-like reaction systems at circum-neutral pH. Acta Chimica Slovenica, 50 (4): 619-632.

212. Goellner, V., Armel, V., Zitolo, A., et al. (2015) Degradation by hydrogen peroxide of metalnitrogen-carbon catalysts for oxygen reduction. Journal of the Electrochemical Society, 162 (6): H403-H414.

213. Banham, D., Kishimoto, T., Sato, T., et al. (2017) New insights into non-precious metal catalyst layer designs for proton exchange membrane fuel cells: Improving performance and stability. Journal of Power Sources, 344: 39-45.

214. Schulenburg, H., Stankov, S., Schünemann, V., et al. (2003) Catalysts for the oxygen reduction from heat-treated iron(III) tetramethoxyphenylporphyrin chloride: Structure and stability and active sites. The Journal of Physical Chemistry B, 107 (34): 9034-9041.

215. Kumar, K., Asset, T., Li, X., et al. (2021) Fe-N-C electrocatalysts' durability: Effects of single atoms' mobility and clustering. ACS Catalysis, 11 (2): 484-494.

216. Domínguez, C., Peña, M.A., Rojas, S., et al. (2016) Effect of the pyrolysis atmosphere and nature of iron precursor on the structure and activity of $\mathrm{Fe} / \mathrm{N}$ based electrocatalysts for the oxygen reduction reaction. International Journal of Hydrogen Energy, 41 (47): 22560-22569.

217. Charreteur, F., Jaouen, F., Dodelet, J.-P. (2009) Iron porphyrin-based cathode catalysts for PEM fuel cells: Influence of pyrolysis gas on activity and stability. Electrochimica Acta, 54 (26): 6622-6630.

218. Osmieri, L., Cullen, D.A., Chung, H.T., et al. (2020) Durability evaluation of a Fe-N-C catalyst in polymer electrolyte fuel cell environment via accelerated stress tests. Nano Energy, 78: 105209.

219. Chenitz, R., Kramm, U.I., Lefèvre, M., et al. (2018) A specific demetalation of Fe-N 4 catalytic sites in the micropores of $\mathrm{NC}-\mathrm{Ar}+\mathrm{NH}_{3}$ is at the origin of the initial activity loss of the highly active $\mathrm{Fe} / \mathrm{N} / \mathrm{C}$ catalyst used for the reduction of oxygen in PEM fuel cells. Energy and Environmental Science, 11 (2): 365-382. 

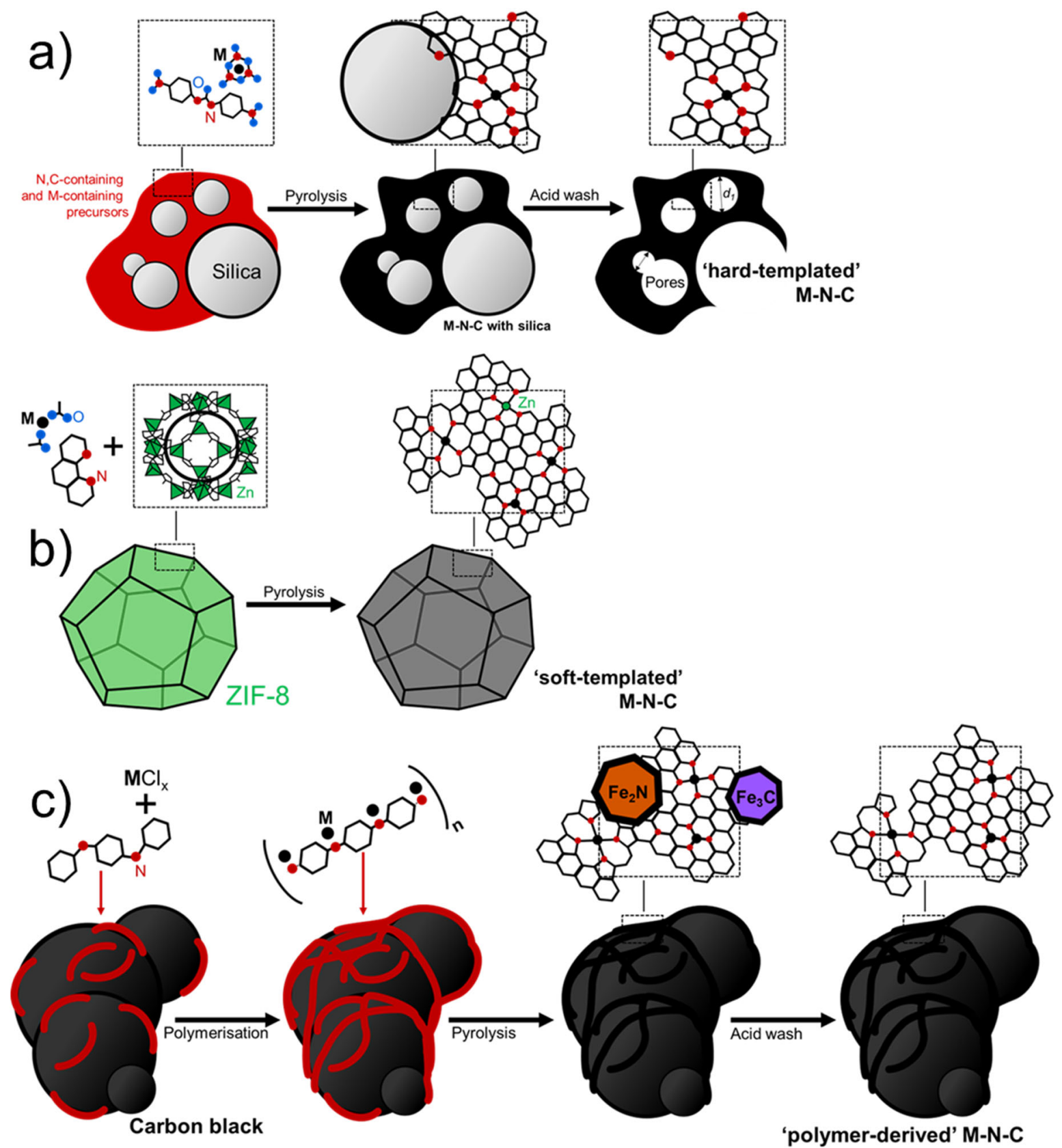

Figure 1. Main synthesis methods to achieve metal-nitrogen-carbon electrocatalysts: a) hard templating approach with silica as an example; b) soft-templating approach with ZIF-8 as an example; c) sacrificial polymer approach with polyaniline as an example - the red lines are representative of the aniline before and after polymerisation. Source: Tristan Asset, Frédéric Maillard, Frédéric Jaouen. 


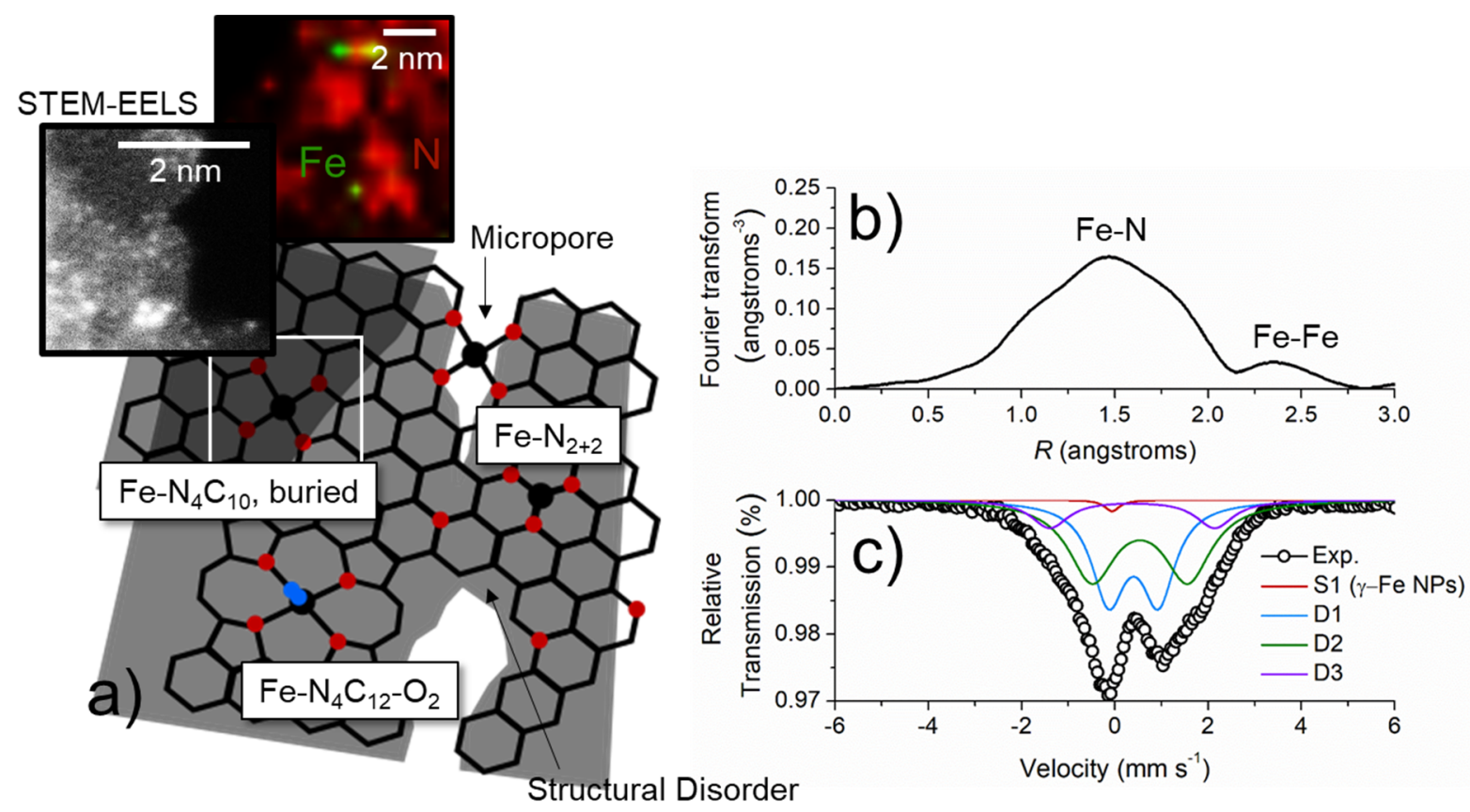

Figure 2. Illustration of the Fe-moieties local coordination and of the experimental tools used to assess their existence. a) graphical representation of a disordered graphene plane, with an illustration of the various metal-containing moieties observed in metal-nitrogen-carbon electrocatalyst and a micrograph of iron atoms embedded in the carbon structure along with an elemental map distribution (the micrograph and the elemental map do not correspond to the same region); b) Fourier transform of the extended X-ray absorption fine structure obtained for an Fe-N-C electrocatalyst, with the main features and c) Mössbauer experimental data and fitting with a singlet component (corresponding to $\gamma$-Fe particles) and three doublets corresponding to different Fe- $\mathrm{N}_{\mathrm{x}}$ SMA sites. Source: Tristan Asset, Frédéric Maillard, Frédéric Jaouen. 


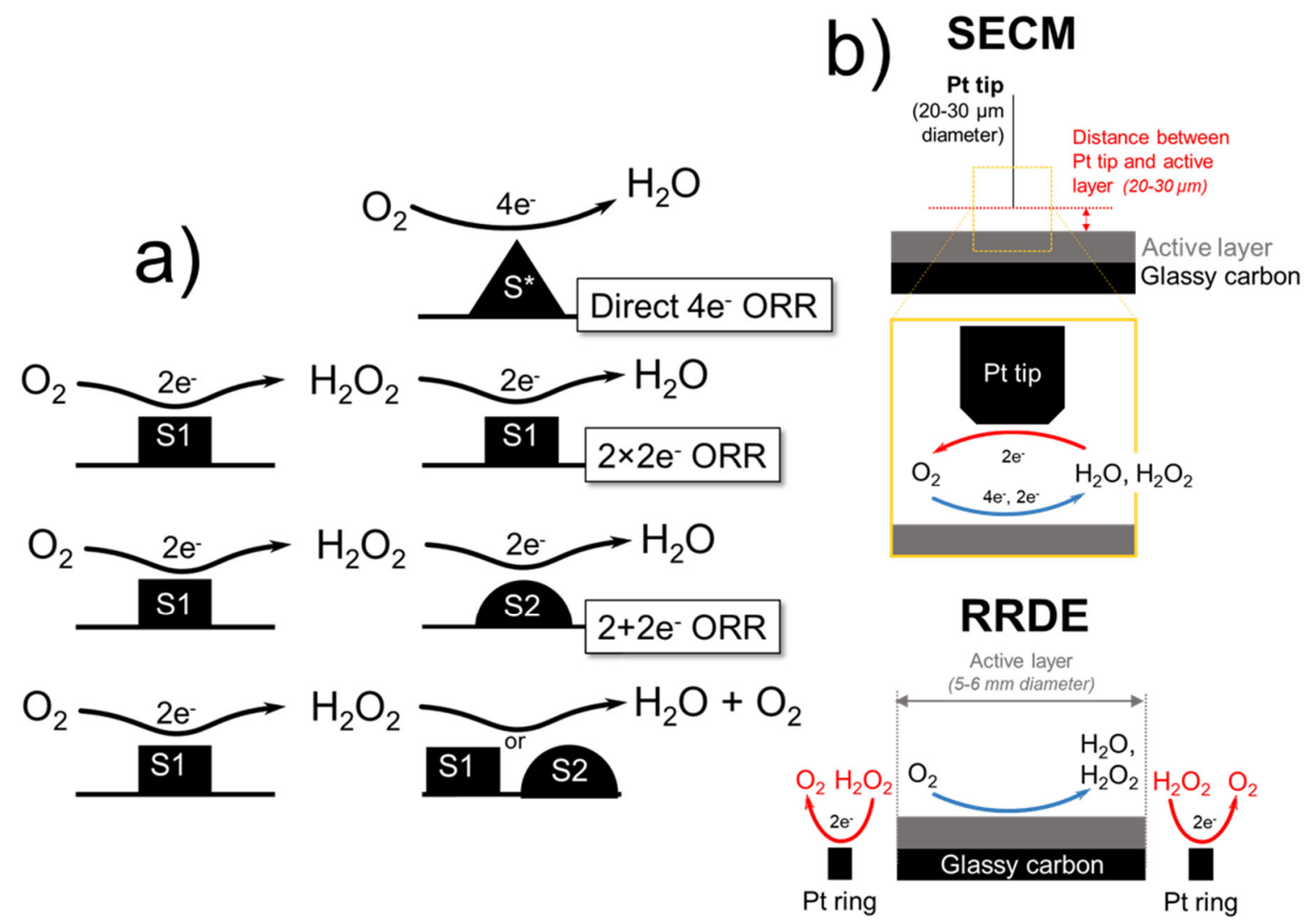

Figure 3. Illustration of the mechanistic and instrumental concepts involved in the study of the oxygen reduction reaction on metal-nitrogen-carbon electrocatalysts. a) Direct and indirect oxygen reduction reaction (ORR) mechanisms and b) scheme of scanning electrochemical microscopy (SECM) and rotating ring disk electrode (RRDE) techniques that can measure the selectivity of electrocatalysts during ORR. Source: Tristan Asset, Frédéric Maillard, Frédéric Jaouen. 

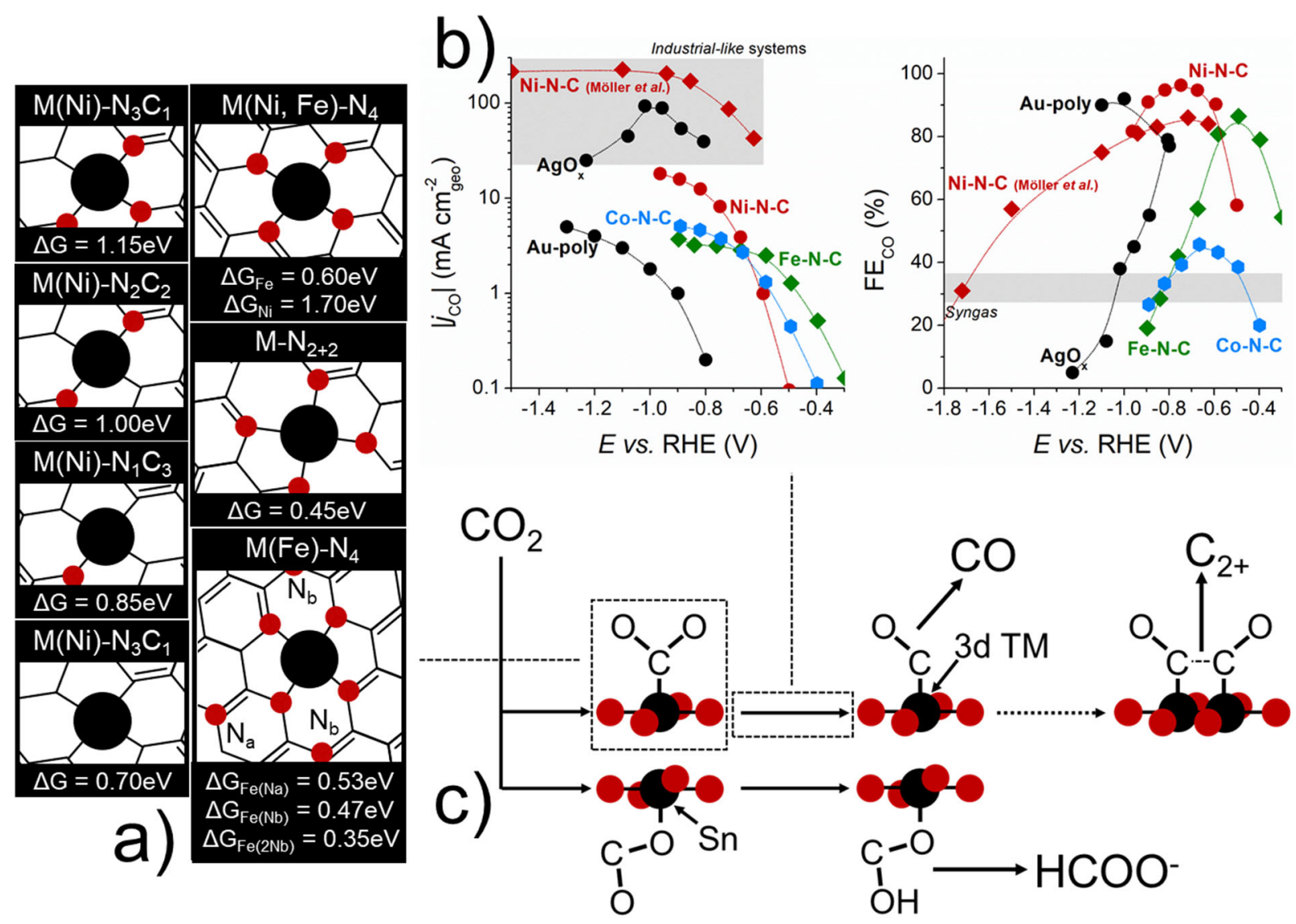

Figure 4. Nature of the active sites, activity and reaction paths for the electrochemical reduction of $\mathrm{CO}_{2}$ by metal-nitrogen-carbon catalysts. (a) illustration of various Metal- $\mathrm{N}_{\mathrm{x}}$ moieties investigated by DFT and the free energy transition for the first proton + electron transfer $\left(\mathrm{CO}_{2} \rightarrow \mathrm{COOH}_{\mathrm{ads}}\right)$; (b) partial current density for $\mathrm{CO}$ generation and faradaic efficiency for $\mathrm{CO}$ generation for various MetalN-C electrocatalysts originating from the work of Pan et al. [145] and Möller et al. [146], along with polycrystalline $\mathrm{Au}$ (Hori et al. [147]) and $\mathrm{AgO}_{\mathrm{x}}$ (Möller et al. [146]) and (c) illustration of the mechanistic pathway on early transition $3 \mathrm{~d}$ Metal-N-C and $\mathrm{Sn}-\mathrm{N}-\mathrm{C}$ for the electrochemical reduction of $\mathrm{CO}_{2}$, along with an hypothetical pathway in presence of dual-atoms moieties. Source: Tristan Asset, Frédéric Maillard, Frédéric Jaouen. 


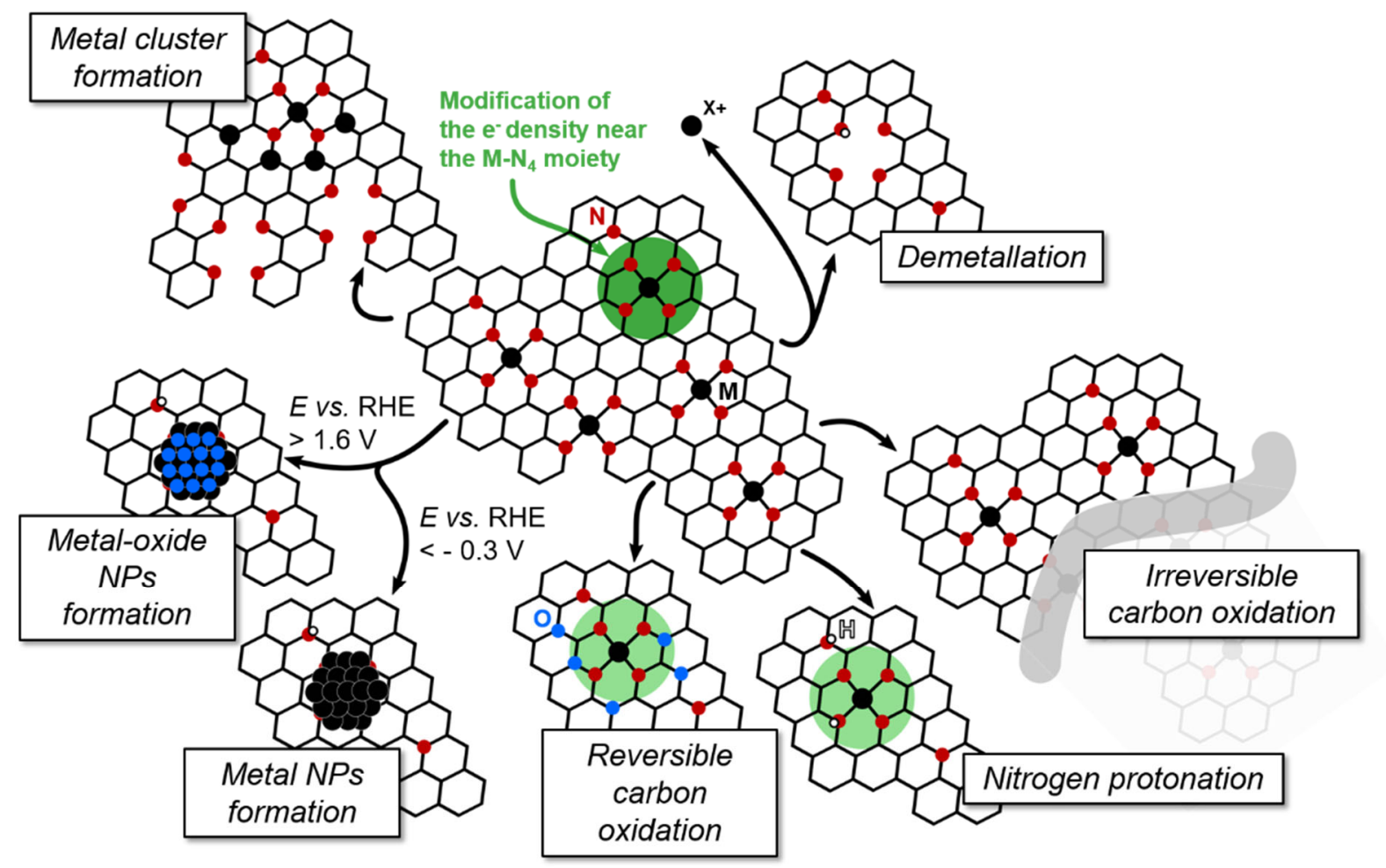

Figure 5. Illustration of the main degradation processes occurring during a Metal-N-C electrocatalyst operation, i.e. demetallation, reversible or irreversible carbon oxidation reaction, nitrogen protonation and metal clusters formation. The intensity of the colour of the green circles is indicative of the electron density near the M-N 4 moiety. Source: Tristan Asset, Frédéric Maillard, Frédéric Jaouen. 